\title{
The Dynamics of Ex-ante Weighted Spread: An Empirical Analysis*
}

4 November 2019

\author{
Georges Dionne $^{a, b} \quad$ Xiaozhou Zhou ${ }^{a, c, \dagger}$
}

\author{
${ }^{a}$ Canada Research Chair in Risk Management, HEC Montréal, Montréal H3T 2A7, Canada \\ ${ }^{b}$ Finance Department, HEC Montréal, Montréal H3T 2A7, Canada \\ ${ }^{c}$ Faculty of Management (ESG), University of Quebec at Montreal, H3C 3P8, Canada
}

Abstract

We model the evolution of the ex-ante weighted spread (EWS) embedded in an open Limit Order Book (LOB) and investigate the impact of observed market-related variables on the spread. Our modeling involves decomposing the joint distribution of the weighted spread into simple and interpretable distributions. Our main results have several implications: (i) EWS features high persistence in autocorrelation; (ii) lower-level LOB remains liquid even after a high trade imbalance; (iii) lower- and higher-level LOB react to temporal spread change and trade imbalance in different ways; and (iv) both trade durations and quote durations have seasonality effects. We also show, through a simple high frequency trading exercise, that the use of the model can be economically important. Further, our model provides an estimation of market resilience.

Keywords: Limit order book, Ex-ante weighted spread, Decomposition model, Liquidity, Resilience.

JEL classification: C22 C41 C53 G11

\section{Introduction}

Since its introduction into financial markets, Limit Order Book (LOB) has received considerable attention from academicians, practitioners, and regulators. The state of the LOB reflects two fundamental elements in finance: liquidity and information. The shape of the LOB is a concrete

\footnotetext{
${ }^{*}$ We thank Yann Bilodeau for his help in constructing the dataset and comments. We also thank Tolga Cenesizoglu, Thierry Foucault, Christian Gourieroux, Gabriel Yergeau, the editor, two anonymous referees, and participants at the 2016 Portsmouth- Fordham Financial conference, 2017 China Meeting of the Econometric Society and the 27th Asian FA Annual Meeting for their remarks. This work was supported by the Social Sciences and Humanities Research Council in Canada [435-2012-1503]; the Canada Foundation for Innovation [219145]; and the Fonds de Recherche sur la société et la culture de Québec [149001].

${ }^{\dagger}$ Corresponding author at: Faculty of Management (ESG), University of Quebec at Montreal (UQAM), 315 rue Sainte-Catherine Est Montreal, Quebec, Canada, H2X 3X2. Tel.: +1 5149873000 ext 0999. E-mail addresses: georges.dionne@hec.ca (G. Dionne), zhou.xiaozhou@uqam.ca (X. Zhou).
} 
form of forward-looking liquidity for traders who seek immediacy. As noted by Amihud and Mendelson (1986), illiquidity can be measured by the cost of immediate execution. In a traditional dealer market, each stock has one designated liquidity provider whose quoting strategy is not disclosed to investors ex-ante. In this context, the cost of immediate execution can only be ex-post deduced from the transactions. However, the extensive use of electronic limit order markets by a large number of exchanges grants investors access to information about ex-ante liquidity, which is determined by price schedules and the corresponding available volumes in the LOB (Glosten (1994), Jain (2005)). As a result, the cost of immediate execution is (partially) visible $^{1}$ and measurable before transactions. Recent studies related to ex-ante liquidity include Irvine et al. (2000), Coppejans et al. (2004), Domowitz et al. (2005), Giot and Grammig (2006), Beltran-Lopez et al. (2009), and Beltran-Lopez et al. (2011). According to Aitken and Comerton-Forde (2003), ex-post liquidity measures involve trade-based measures, while ex-ante liquidity measures are order-based. The former measures are the most widely used and indicate what the traders have obtained in the realized transaction. The second group captures the cost related to potential immediate trading.

The shape of the LOB is also an outcome of limit orders from both informed and uninformed traders. Earlier theoretical models assume that the LOB is solely constructed by limit orders submitted by uninformed traders and that market orders contain information. ${ }^{2}$ Consequently, the shape of the LOB is determined by uninformed liquidity providers who have to protect themselves from traders with superior information. However, recent theoretical models (Parlour (1998), Foucault et al. (2005), Goettler et al. (2009)) allow informed traders to submit both market orders and limit orders, and conclude that informed traders do use limit orders in their strategy design. As a result, the state of the LOB can better predict future price. The existence of this prediction power is analyzed by a large number of empirical studies (Cao et al. (2008), Kalay and Wohl (2009), Pascual and Veredas (2010), Cenesizoglu et al. (2018), among others). In general, they argue that state of the LOB, which is characterized by variables such as depth, slope, convexity and imbalance, has prediction power on either short-term price movement or volatility.

Given the importance of the LOB in price formation and in gauging liquidity and information asymmetry, the questions of how the LOB evolves and what determines the dynamics of

\footnotetext{
${ }^{1}$ Because most exchanges allow also iceberg and hidden orders. In addition, due to the latency, the realized cost at execution could be different from the expected cost based on the information of the LOB before submitting market orders. We thank an anonymous referee for pointing out the importance of latency.

${ }^{2}$ See Kyle (1985), Glosten and Milgrom (1985), and Glosten (1994).
} 
the LOB are important but still open. To fill this gap, this paper focuses on the dynamics of the ex-ante weighted spread (EWS). Typically, this weighted spread is a measure of the state of the LOB. Compared with the widely used best bid-ask spread, ex-ante weighted spread provides a more complete picture of the shape of the LOB and captures more information embedded in it. Theoretical models attempt to explain the shape of the LOB with various sources. One class of theoretical models takes information asymmetry as the determinant of the shape of the LOB (Rock (1990), Glosten (1994), Goettler et al. (2005), and Goettler et al. (2009)). In another category of theoretical models with the absence of asymmetric information, nonexecution probability, waiting costs (traders' patience), as well as competition among traders are determinant factors of the shape of the LOB (Foucault (1999), Foucault et al. (2005), and Rosu (2009)). Moreover, the shape of the LOB is also highly affected by high-frequency trading (HFT) strategies. As shown by Hendershott and Riordan (2013), for stocks of DAX30, high-frequency traders represent $52 \%$ of market order volume and $64 \%$ of nonmarketable limit order volume. Hagstromer and Norden (2013) examine data from NASDAQ-OMX Stockholm and find that market makers constitute the lion's share of HFT trading volume (65\%-71\%). Other factors that probably influence the shape of the LOB are particular setups of trading mechanism and regulatory issues. Riordan and Storkenmaier (2012) examine the effect of a technological upgrade on the market liquidity of 98 actively traded German stocks, and show that both effective spreads and average price impacts drop with the upgrade. Brogaard et al. (2015) find that liquidity improves for the overall market after introduction of colocation services.

Instead of theoretically identifying the determinants of the shape of the LOB, this paper attempts to empirically capture a more general and realistic LOB evolution pattern that is much more complete than that characterized by structural models. Specifically, this study models the ex-ante weighted spread (EWS) using a tractable decomposition model that allows for various factors in a flexible way, and tests several empirical implications stemming from theoretical models. Furthermore, we attempt to quantify the effect of market-related high frequency variables on these factors. To do so, we construct and model the ex-ante weighted spread to capture the dynamics of the lower-level and higher-level LOB. Having found that our model can capture the dynamics of EWS effectively, we then show, through a simple high frequency trading exercise for all stocks from our sample, that the use of the model can also lead to economic gains. Finally, our model allows for practitioners to get an estimation of resiliency. Market resiliency is an important dynamic phenomenon that has received little attention in empirical studies. Market conditions with large spreads are less liquid and less resilient (Foucault et al. (2005)). Having a 
good measure of the ex-ante weighted spread can be instrumental to estimate market resiliency and obtain a good predictor of trading aggressivity.

To our knowledge, we are the first to consider modeling the ex-ante weighted spread using a decomposition model and including a large set of factors. Given the particularity of UHF data and the complexity of microstructure analysis, one possible modeling framework involves consistently decomposing the joint distribution of a target variable into simple and interpretable distributions. The idea of decomposition was pioneered by Rogers and Zane (1998), and aims at constructing observation-driven models in the sense of Cox et al. (1981). The decomposition model was first used to analyze transaction price dynamics. Hausman et al. (1992) and Russell and Engle (2005) propose an Autoregressive Conditional Multinomial (ACM) and ordered Probit model respectively. Rydberg and Shephard (2003) achieve the same goals by decomposing the joint distribution of tick-by-tick transaction price changes into three sequential components. And the decomposition model permits to predict price movements or price level with the help of simulations. McCulloch and Tsay (2001) model the transaction price variation process using the decomposition model. In their framework, they initialize a price variation and duration (PCD) model that decomposes the price variations into four factors, and introduce time and liquidity dimensions in modeling price variation dynamics. The duration between two consecutive transactions and the number of trades during this duration are modeled as implicit factors for the price changes. In total, they use six conditional models to capture the dynamics of price changes. Manganelli (2005) applies the decomposition methodology when investigating the simultaneous interaction between duration, volume and return. Two subgroups, classified by trade intensity, perform different dynamics. The decomposition framework remains flexible for more complicated modeling. Depending on different modeling assumptions, addition or deletion of certain factors is possible.

Our paper differs from the literature in several respects. First, instead of aggregating the time for a fixed interval, our analysis contains a time dimension. Specifically, our paper models the dynamics of tick-by-tick liquidity, and takes trade and quote durations as a proxy of trading activities, and investigates their roles in explaining the dynamics of open LOB. It is widely known that one important feature of the order-driven market involves the use of high-speed computers and advanced algorithms. As a result, the trading frequency shrinks from the time frame of minutes to the time scale of microseconds (even nanoseconds in some exchanges). By modeling data at tick-by-tick frequency, we attempt to take into account all available trading and quoting information. It is widely recognized that trade can convey private information and 
have an impact on the quote. Hasbrouck (1991) shows that, in an order-driven market, the price impact of trades is positive, and large trades cause the spread to widen. Intuitively, when facing informed traders, market makers protect themselves by quickly widening the spread and closely monitoring market order arrivals. Recently, the studies of Baruch et al. (2017) and Brogaard et al. (2019) show that limit orders play an important role in price discovery regardless of the existence of trading. Therefore, both trade duration and quote duration could be relevant measures for information flow or market dynamics. In addition, regarding model implementation, once the model is estimated on tick-by-tick frequency, we can test the model performance and compute EWS by Monte Carlo simulation for any horizon without re-estimating the model.

Second, by applying a decomposition model, we perform a much finer analysis of ex-ante weighted spread and take advantage of econometric modeling by attempting to capture a more general and realistic LOB trading pattern that is much more complete than that characterized by structural models. Specifically, following Engle and Lunde (2003) and Rydberg and Shephard (2003), we use different factors to model the dynamics of weighted spread changes. ${ }^{3}$ Our main objective is to answer the question of how information sets of limit order traders are updated after trading and quoting activities by identifying the possible determinants of each factor from a set of market-related variables. Our empirical findings not only test several empirical implications derived from theoretical models but also offer guidance for new theoretical models in market microstructure. Practically, our modeling is also greatly useful for the Smart Order Routing (SOR) system ${ }^{4}$ or for large financial institutions that participate in the market as real-time market makers.

Third, regarding the explanatory variables, in addition to the lagged dependent variables, we also include various market-related variables in the different factor equations. Whereas most papers take one variable as the explanatory variable and suppose that this variable can summarize all the trade information, our variables are volume-related, duration-related and trade imbalance related. Among these variables, we also distinguish between the short-run and long-run ${ }^{5}$ variables to reflect their time dimension. Our results provide several conlusions: First, to model the dynamics of EWS, it is essential to include the lagged auto-dependent structure to capture

\footnotetext{
${ }^{3}$ The factors used to model the dynamics of the EWT include trade duration, quote duration, activity, direction, and size factors.

${ }^{4}$ Smart Order Router is a system designed to submit orders in the best available way by relying on the market condition and defined rules. Usually, SOR searches for the best execution price across fragmented markets. In this paper, we use SOR to refer to the execution practice that has the same objective as a general SOR but is applied to the temporal dimension (over the course of the trading day).

${ }^{5}$ Short-run variables are variables at a given time point, whereas long-run variables summarize the information over an interval.
} 
the high persistence of autocorrelation; Second, most market-related variables have significant impacts on the dynamics of EWS; Third, lower- and higher-level LOB react to temporal spread change and trade imbalance in different ways; Fourth, the trade durations and quote durations have an obvious seasonality pattern, whereas the seasonality pattern for other factors is much weaker.

The rest of the paper is organized as follows: Section 2 describes the Xetra trading system and the ex-ante weighted spread modeled in this study. Section 3 presents the decomposition model and the market-related variables used to explain the dynamics of each factor. Section 4 applies our econometric model and reports the results of estimation, and in-sample and out-of-sample tests. Section 5 shows, through a simple high-frequency trading exercise, that the information captured by our model is also economically important. Section 6 provides an application of our decomposition model to resilience estimation. Section 7 concludes the paper and proposes new research directions.

\section{Xetra Trading System and Ex-ante Weighted Spread}

\subsection{Xetra Trading System}

Electronic trading systems have been adopted by many stock exchanges during the last two decades. The data used in this study are from the Xetra trading system, which is operated by Deutsche Börse at the Frankfurt Stock Exchange (FSE) and has a similar structure to the Integrated Single Book of NASDAQ and Super Dot of NYSE. The Xetra trading system realizes more than $95 \%$ of the total transactions at German exchanges. In this study, we focus on continuous trading.

During continuous trading, ${ }^{6}$ there are no dedicated market makers and all liquidity comes from limit orders in the LOB. The Xetra trading system imposes a Price-Visibility-Time Priority condition, where the electronic trading system places the incoming order after checking the price and timestamps of all available limit orders in the LOB. Our database includes 20 levels of LOB information, ${ }^{7}$ which means that, by monitoring the LOB, any registered member can

\footnotetext{
${ }^{6}$ There are two types of trading mechanisms during normal trading hours: call auction and continuous auction. A call auction can be organized once or several times during the trading day in which the clearance price is determined by the state of the LOB and remains as the open price for the following continuous auction.

${ }^{7}$ Fully hidden orders and the hidden part of an iceberg order are not observable in our dataset. However, as we observe the state of the LOB before and after the transaction, we can evaluate if a market order hits hidden orders or not. Our backtest results show that fewer than $3 \%$ of the market orders run into hidden orders, which represents about $6 \%$ of trade volumes. The presence of hidden orders makes our EWS slightly underestimating the actual liquidity. We thank one anonymous referee for pointing out the impact of hidden orders on the EWS.
} 
evaluate the liquidity supply dynamics and potential price impact of a market order. However, there is no information on the identities of market participants. A more detailed description of the reconstruction of the LOB is available in the online appendix.

\subsection{Ex-ante Weighted Spread}

Ex-ante weighted spread is an instantaneous round-trip relative price impact for a given trade size. In this study, we define the ex-ante weighted spread as follows:

$$
\begin{gathered}
E W S^{q}=\frac{P_{n e t, b u y}^{q}-P_{n e t, s e l l}^{q}}{P_{\text {mid }}} \times 10000, \\
P_{n e t, \text { buy }}^{q}=\frac{\sum_{k=1}^{K-1} P_{k, i} \cdot v_{k, i}+P_{K, i} \cdot v_{K, i}}{v} \text { and } v_{K, i}=v-\sum_{k=1}^{K-1} v_{k, i},
\end{gathered}
$$

where $q$ is the potential size in Euros, ${ }^{8} P_{n e t, b u y}^{q}$ is the average price when a buy market order of $q$ Euros arrives and $P_{n e t, \text { sell }}^{q}$ relates to the average price for a sell market order of $q$ Euros. $v$ is the total volume bought by a market order of $q$ Euros. $P_{k, i}$ and $v_{k, i}$ are the $k$ th level ask price and volume available, respectively. $v_{K, i}$ is the quantity left after $K-1$ levels are completely consumed by the market order of $q$ Euros. $P_{n e t, \text { sell }}^{q}$ is computed in a similar way. $P_{m i d}$ is the mid-quote of the bid-ask spread. Intuitively, it is also the cost in basis points of an immediate demand for liquidity from buy and sell market orders. For example, an EWS of 10 basis points related to a market order of 25,000 Euros means that the cost (or spread) caused by, simultaneously, buying and selling a market order of 25,000 Euros is 25 Euros. By choosing a different volume $q$, we can identify the EWS on the open LOB as shown in Figure 1. For comparison purposes, we adjust $q$ to reflect the lower-level and higher-level LOB for each stock. ${ }^{9}$ The previous market microstructure literature that considers the quantity available in LOB includes Irvine et al. (2000), Domowitz et al. (2005), and Coppejans et al. (2004), among others.

The EWS is based on $P_{\text {mid }}$ and the difference between $P_{n e t, b u y}^{q}$ and $P_{n e t, \text { sell }}^{q}$. Theoretically, there are infinite combinations of $P_{n e t, b u y}^{q}$ and $P_{n e t, s e l l}^{q}$ for the same difference. That is, the spread

\footnotetext{
${ }^{8}$ To avoid the impact of stock price and outstanding shares across different stocks, for each stock, we choose $q$ from its own trade volume distribution.

${ }^{9}$ Given that $q$ is ad-hoc, hereafter, we use the notations $E W S^{L o w}$ and $E W S^{H i g h}$ for low-level spread and high-level spread, respectively. In addition, we keep $E W S^{q}$ as a general term for ex-ante weighted spread.
} 
may come from either side or both sides of the LOB. However, our study focuses on stocks' global spread. For both buy-side and sell-side, the corresponding one-side EWS can be defined and computed in a similar fashion. Figure 2 presents the average EWS as a function of $q$ for 30 stocks in DAX30 for May 2011.

[Insert Figure 1 here]

[Insert Figure 2 here]

The EWS closely relates to Kyle's (1985) liquidity criteria: tightness, depth, and resilience. Specifically, the EWS is a measurement of the instantaneous depth-dependent tightness. Compared with the widely used bid-ask spread, the EWS is a more sensible measure because it considers both price and volume. Further, our model captures the evolution of the EWS, which corresponds to resilience. Practically, the EWS also can be used for several ends: first, it can help decision making in security selection when constructing a portfolio. Among the stocks with the same correlation with the market portfolio, a small EWS stock will decrease the trading cost and ultimately provide a higher net return. Second, the EWS can also be used for comparison purposes. For instance, a cross-listing stock may have different liquidity features in different markets. By using the EWS, one can quantify this difference by choosing a given volume.

\section{Methodology}

\subsection{Model}

To better capture the dynamics of EWS, we first include a time dimension that provides information on trading intensity. Following Engle and Lunde (2003), we consider trade and quote as a bivariate point process. Based on the timestamps of these point processes, we can define two types of duration: trade duration and quote duration, which constitute a bivariate duration process. However, due to the non-synchronization problem shown in Figure 3, we further assume that the trade times are the initiators for both the following trade and the next quote update. ${ }^{10}$ Consequently, trade durations and quote durations with the same index share the same original timestamp ${ }^{11}$. The economic intuition behind the assumption is that the limit order traders in

\footnotetext{
${ }^{10} \mathrm{Lu}$ and Abergel (2018) show that trades are more likely to be the driving force of the trade-quote and LOB dynamics.

${ }^{11}$ We also estimate the model by supposing that the quote updates initiate the dynamics. The results remain similar.
} 
open LOB update their quotes by observing transactions. After each transaction, we compute the quote duration based on the very last transaction.

[Insert Figure 3 here]

As mentioned by Engle and Lunde (2003), by taking the transaction times as the origin of each pair of durations, two possible situations may occur for quote duration: an uncensored observation or a censored observation. The uncensored duration is when the quote update occurs before the next trade arrival and the censored duration happens when the following trade arrives before the quote update. We denote $x_{i}$ and $y_{i}$ as the trade duration and quote duration, respectively, and further define the observed quote duration $\tilde{y}_{i}=\left(1-d_{i}\right) \cdot y_{i}+d_{i} x_{i}$, where $d_{i}=I_{\left\{y_{i}>x_{i}\right\}}$.

Apart from the time dimension, we use the above-mentioned EWS as the measure for the state of the LOB. In the uncensored situation, we take the average of the measure within the first quote update timestamp and the following trade timestamp. Its evolution can be written as:

$$
E W S_{i}^{q}=E W S_{0}^{q}+\sum_{k=1}^{i} Z_{k}
$$

where $Z_{k}$ is defined as the $k$ th rounded signed change in $E W S^{q}$. In our dataset, $Z_{k}$ is stationary and the stationarity is tested by the augmented Dickey-Fuller test. ${ }^{12}$

We define $p$ as the joint density for trade duration, quote duration and $E W S^{q}$ changes. We propose the following decomposition for this joint density of the $k$ th mark:

$p\left(x_{k}, \tilde{y_{k}}, z_{k}^{q} \mid F_{k-1} ; \omega\right)$

$=g\left(x_{k} \mid F_{k-1} ; \omega_{1}\right) \cdot f^{D u r}\left(\tilde{y_{k}} \mid x_{k}, F_{k-1} ; \omega_{2}\right) \cdot f^{A}\left(A_{k} \mid x_{k}, \tilde{y_{k}}, F_{k-1} ; \omega_{3}\right)$.

$f^{D}\left(D_{k} \mid x_{k}, \tilde{y_{k}}, A_{k}, F_{k-1} ; \omega_{4}\right) \cdot f^{S}\left(S_{k} \mid x_{k}, \tilde{y_{k}}, A_{k}, D_{k}, F_{k-1} ; \omega_{5}\right)$.

We define $x_{k}$ and $\tilde{y_{k}}$ as duration factors, which relate to the trade duration and observed quote duration, for $Z_{k}$. Conditional on information set $F_{k-1}$ and two durations, $A_{k}$ takes the value of 0 or 1 , which indicates if there is a change in the $k$ th $E W S^{q}$. Conditional on $A_{k}=1, D_{k}$ relates to the direction of the $E W S^{q}$ change by taking on the value -1 and +1 . Finally, given the information set $\left(F_{k-1}, A_{k}=1, D_{k}\right), S_{k}$ takes on positive integers and indicates the size of the change. $\omega$ relates to the parameter set including $\omega_{1}, \omega_{2}, \omega_{3}, \omega_{4}$, and $\omega_{5}$, which are the parameters for factors of trade duration, observed quote duration, activity, direction and size.

\footnotetext{
${ }^{12}$ The augmented Dickey-Fuller test rejects the hypothesis of a unit root for $Z_{k}$ for all stocks in our sample. For the sake of brevity, we do not present our test results here. They are available from authors.
} 
The motivation for our choice of factors is as follows. A tractable decomposition model allows for simple, observable, flexible, and interpretable factors. We decompose the $Z_{k}$ into the activity, direction, and size factors because they capture different facets of a single change in $E W S^{q}$. The first factor, activity, measures if $E W S^{q}$ will change or not after a trade. The second factor, direction, provides information on increase or decrease in the following $E W S^{q}$ if there is a change. Finally, the factor of size assesses the magnitude of this change. Further, as shown below, we choose the auto-logistic model for the activity and direction factors, and the geometric model for the size factor because these models are concave, and numerical optimization can be done easily and reliably.

\subsection{Models for Temporal Factors}

We adopt the Log-ACD model originally introduced by Bauwens and Giot (2000) in modeling the irregularly spaced trade durations, which represents a main characteristic of high frequency data:

$$
\begin{gathered}
\frac{\mathrm{x}_{\mathrm{k}}}{\psi_{\mathrm{k}}}=\varepsilon_{\mathrm{k}}, \\
\psi_{k}=\exp \left(\omega+\sum_{j=1}^{p} \alpha_{j} \varepsilon_{k-j}+\sum_{j=1}^{l} \beta_{j} \ln \psi_{k-j}+\Psi^{\prime} W_{k-1}\right),
\end{gathered}
$$

where $\psi_{k}=E\left(x_{k} \mid F_{k-1}\right)$ and $\varepsilon_{k}$ is a i.i.d. random variable following the generalized gamma distribution with unit expectation. The process of duration is composed of a sequence of deseasonalized durations. $W_{k-1}$ is a vector of market-related variables available at $k-1$, which includes market-related variables and quote-related variables. We use the Log-ACD because it is more flexible in modeling, and the positivity constraint on durations is always respected. ${ }^{13}$ We adopt a similar structure for observed quote duration, that is

$$
\begin{gathered}
\frac{\tilde{y_{\mathrm{k}}}}{\phi_{\mathrm{k}}}=\epsilon_{\mathrm{k}}, \\
\phi_{k}=\exp \left(\mu+\sum_{j=1}^{p} \rho_{j} \epsilon_{k-j}+\rho_{j+1} \epsilon_{k-1} d_{k-1}+\sum_{j=1}^{l} \delta_{j} \ln \phi_{k-j}+\Phi^{\prime} V_{k-1}\right),
\end{gathered}
$$

\footnotetext{
${ }^{13}$ The ACD (Autoregression Conditional Duration) model is proposed by Engle and Russell (1998); it is widely used for duration modeling.
} 
where $\phi_{k}=E\left(\tilde{y_{k}} \mid F_{k-1}\right), \epsilon_{k}$ is supposed to be i.i.d exponential distributed and the error distribution is supported by the estimation convergence. In the equation, we add a term with a censored dummy variable to capture the impact of censored observation and the vector of market-related variables $V_{k-1}$, which may have some common variables with $W_{k-1}$. We show in the following section that because quote durations are conditional on transactions, one possible determinant variable could be the expected trade duration available at tick $k$.

\subsection{Models for the Activity, Direction, and Size Factors}

We next turn to explain the modeling for the activity, direction, and size factors. In our dataset, these three factors are all stationary, which is confirmed by the augmented Dickey-Fuller test. ${ }^{14}$ The dynamics of each stationary factor are modeled by an autoregressive structure and market condition variables. We include the autoregressive part because $Z_{k}$ and all three factors feature a high persistence in autocorrelation. More specifically, we adopt a structure called the GLARMA (Generalized Linear Autoregressive Moving Average) binary model, which is a generalized structure of auto-logistic structure allowing for moving average-type behavior (Shephard (1995)). The use of market condition variables is straightforward in that liquidity is determined or driven by market conditions.

Activity is a bivariate variable that takes the values of 0 or 1 to indicate whether there is a change in the $E W S^{q}$. To this end, we use the auto-logistic model (Cox et al., (1981)). Given that the log-likelihood function of the auto-logistic model is concave, numerical optimization can be done easily and reliably. However, the high-frequency data often exhibit a slow decay for longer lags in an autoregressive structure. Thus there is a trade-off between bias and variance, i.e., inference with too few parameters may be biased, while that with too many parameters may cause precision and identification problems. To solve this, the auto-logistic model for activity is defined as:

$$
\begin{aligned}
& f\left(A_{k}=1 \mid F_{k-1}, x_{k}, \tilde{y_{k}}\right)=p\left(\theta_{k}^{A}\right), \text { where } p\left(\theta_{k}^{A}\right)=\frac{\exp \left(\theta_{k}^{A}\right)}{1+\exp \left(\theta_{k}^{A}\right)} \\
& \text { and } \theta_{k}^{A}=\Pi_{A}^{\prime} M_{k-1}^{A}+g_{k}^{A}, \quad g_{k}^{A}=\sum_{j=1}^{p} \gamma_{j}^{A} g_{k-j}^{A}+\sum_{j=1}^{l} \lambda_{j}^{A} A_{k-j} .
\end{aligned}
$$

\footnotetext{
${ }^{14}$ The augmented Dickey-Fuller test rejects the hypothesis of a unit root for the activity, direction, and size factors for all stocks in our sample. For the sake of brevity, we do not present our test results. But they are available from authors.
} 


$$
\text { Consequently, } f\left(A_{k}=0 \mid F_{k-1}, x_{k}, \tilde{y_{k}}\right)=\frac{1}{1+\exp \left(\theta_{k}^{A}\right)} \text {, }
$$

where $M_{k-1}^{A}$ is the vector of market-related variables for the activity factor known at $k-1$. In this logistic modeling, the parameter $\theta_{k}^{A}$ is time-varying and depends on both its own lag variables, such as lags of $g_{k}$ and $A_{k}$, and some market-related variables. The model will be first validated by applying the Ljung-Box test on the standardized errors defined by:

$$
u_{k}^{A}=\frac{A_{k}-p\left(\theta_{k}^{A}\right)}{\sqrt{p\left(\theta_{k}^{A}\right)\left(1-p\left(\theta_{k}^{A}\right)\right)}},
$$

which should be uncorrelated with zero mean and unit variance. Moreover, we evaluate the model's performance by using the McFadden's pseudo-R squared, the Receiver operating characteristic (ROC) and Count accuranry. ${ }^{15}$

In a similar way, the direction factor, conditional on the activity factor, is specified by another binary process on +1 or -1 ( +1 means that spread becomes larger, and -1 is related to a smaller spread for trading volume $q$ ) and is estimated by another auto-logistic model:

$$
\begin{gathered}
f\left(D_{k}=1 \mid F_{k-1}, x_{k}, \tilde{y_{k}}, A_{k}=1\right)=p\left(\theta_{k}^{D}\right), \text { where } p\left(\theta_{k}^{D}\right)=\frac{\exp \left(\theta_{k}^{D}\right)}{1+\exp \left(\theta_{k}^{D}\right)} \\
\quad \text { and } \theta_{k}^{D}=\Pi_{D^{\prime}}^{\prime} M_{k-1}^{D}+g_{k}^{D}, \quad g_{k}^{D}=\sum_{j=1}^{p} \gamma_{j}^{D} g_{k-j}^{D}+\sum_{j=1}^{l} \lambda_{j}^{D} D_{k-j}, \\
\text { Consequently, } f\left(D_{k}=-1 \mid F_{k-1}, x_{k}, \tilde{y_{k}}, A_{k}=1\right)=\frac{1}{1+\exp \left(\theta_{k}^{D}\right)} .
\end{gathered}
$$

$M_{k}^{D}$ is a vector including market-related variables of subset $F_{k-1}$ and $\Pi^{D}$ is a parameter vector. Note that the vectors $M^{D}$ and $M^{A}$ might have some identical market-related variables.

Once the model is estimated, we use the Ljung-Box test to validate its ability to capture the main features of the data. The test will be applied to standardized residuals:

$$
u_{k}^{D}=\frac{D_{k}-\left(2 p\left(\theta_{k}^{D}\right)-1\right)}{2 \sqrt{p\left(\theta_{k}^{D}\right)\left(1-p\left(\theta_{k}^{D}\right)\right)}} .
$$

The model is also validated by the McFadden's pseudo-R squared, the Receiver Operating Characteristic (ROC) and Count accuracy.

\footnotetext{
${ }^{15}$ The McFadden's R squared measure is defined as $R_{M c F a d e n}^{2}=1-\frac{\log \left(L_{c}\right)}{\log \left(L_{\text {null }}\right)}$ where $L_{c}$ denotes the likelihood value from the current fitted model and $L_{\text {null }}$ denotes the corresponding value for the null model. ROC evaluates binary model accuracy at various threshold settings (Swets 1986, 1988)) by considering Type I and Type II errors. Count accuracy measures the in-sample accuracy predicted by the model.
} 
The last factor is Size, which captures the magnitude of $Z_{k}$. We adopt a geometric process for the size factor because of its simplicity and generality:

$$
\begin{gathered}
S_{k} \mid\left(F_{k-1}, x_{k}, \tilde{y}_{k}, A_{k}=1\right) \sim 1+\mathrm{g}\left(\lambda_{k}\right), \\
\lambda_{k}=\frac{\exp \left(\theta_{k}^{S i z}\right)}{1+\exp \left(\theta_{k}^{\text {Siz }}\right)}, \\
\theta_{k}^{S i z}=\Pi_{S i z}^{\prime} M_{k-1}^{S i z}+g_{k}^{S i z} \text { and } g_{k}^{S i z}=\sum_{j=1}^{p} \gamma_{j}^{S i z} g_{k-j}^{S i z}+\sum_{j=1}^{l} \lambda_{j}^{S i z} S_{k-j},
\end{gathered}
$$

where $M_{k-1}^{S i z}$ is a vector of market-related variables and $\Pi_{S i z}$ is the corresponding parameter vector. $g\left(\lambda_{k}\right)$ indicates the geometric distribution with parameter $\lambda_{k} \cdot{ }^{16}$ In order to capture the asymmetry between up-move size and down-move size, we add a direction variable in the vector of market-related variables. In equation (11), we add 1 to the geometric distribution because the minimum change is 1 . We also apply the Ljung-Box statistics to standardized residuals to evaluate the model. Given the conditional distribution of Size, we have

$$
\begin{gathered}
\left.E\left(S_{k}-1 \mid F_{k-1}, x_{k}, \tilde{y_{k}}, A_{k}=1\right)\right)=\frac{1-\lambda_{k}}{\lambda_{k}}, \\
\left.\operatorname{Var}\left(S_{k}-1 \mid F_{k-1}, x_{k}, \tilde{y_{k}}, A_{k}=1\right)\right)=\frac{1-\lambda_{k}}{\left(\lambda_{k}\right)^{2}} .
\end{gathered}
$$

Standardized residuals are computed as

$$
u_{k}^{S i z}=\frac{S_{k}-1-E\left(S_{k}-1 \mid F_{k-1}, x_{k}, \tilde{y_{k}}, A_{k}=1\right)}{\sqrt{\operatorname{Var}\left(S_{k}-1 \mid F_{k-1}, x_{k}, \tilde{y_{k}}, A_{k}=1\right)}},
$$

and an adequate modeling requires that $u_{k}^{S i z}$ be uncorrelated with zero mean and unit variance. To summarize, for the estimation process, we can estimate each factor separately by using the Maximum Likelihood approach. The BIC criteria will be applied for the model selection, especially for the choice of number of lags. Moreover, to make sure that the model captures the main features of the data, we perform various tests to evaluate the performance of the models. Then, with the previous specifications, all the observations can be classified into one of the three following categories:

1) There is no change in the EWS, that is, activity factor $A_{k}=0$ and no direction and size factors.

\footnotetext{
${ }^{16}$ The general probability distribution function is $f(x=m)=\lambda(1-\lambda)^{m}, 0<\lambda<1, m=0,1,2, \ldots$
} 
2) Weighted spread increases and the size changes by at least one unit. The corresponding factors are: $A_{k}=1, D_{k}=1$, and $S_{k}=s_{k}$;

3) Weighted spread decreases and the size changes by at least one unit. The corresponding factors are: $A_{k}=1, D_{k}=-1$, and $S_{k}=s_{k}$;

The maximum likelihood estimation function is equal to:

$$
\begin{aligned}
& L\left(\omega_{1}, \omega_{2}, \omega_{3}, \omega_{4}, \omega_{5} ; x_{k}, \tilde{y_{k}}, z_{k}^{q}\right) \\
& =\sum_{k=1}^{n}\left[\begin{array}{l}
\log \left[g\left(x_{k} \mid F_{k-1} ; \omega_{1}\right)\right]+\log \left[f^{D u r}\left(\tilde{y_{k}} \mid x_{k}, F_{k-1} ; \omega_{2}\right)\right] \\
+I_{k}(1) \cdot K 1+I_{k}(2) \cdot K 2+I_{k}(3) \cdot K 3
\end{array}\right],
\end{aligned}
$$

where

$K 1=\log \left(f^{A}\left(A_{k} \mid F_{k-1} ; \omega_{3}\right)\right) ;$

$K 2=\log \left(1-f^{A}\left(A_{k} \mid F_{k-1} ; \omega_{3}\right)\right)+\log \left(f^{D}\left(D_{k} \mid F_{k-1} ; \omega_{4}\right)\right)+\log \left(f^{S}\left(S_{k} \mid F_{k-1} ; \omega_{5}\right)\right) ;$

$K 3=\log \left(1-f^{A}\left(A_{k} \mid F_{k-1} ; \omega_{3}\right)\right)+\log \left(1-f^{D}\left(D_{k} \mid F_{k-1} ; \omega_{4}\right)\right)+\log \left(f^{S}\left(S_{k} \mid F_{k-1} ; \omega_{5}\right)\right)$.

$I_{k}(1), I_{k}(2), I_{k}(3)$ correspond to the indicator function related to the three categories mentioned above.

In sum, the advantage of this modeling is that the partition enables us to simplify the modeling and computation task by specifying the suitable econometric models for the marginal densities of trade duration and conditional densities for quote duration, along with factors such as Activity, Direction and Size. In addition, for different purposes, the model could also be extended to a more or less complicated context by including other factors. In these decomposition models, one of the crucial tasks is to identify the market-related variables.

\subsection{Market-related Variables Set}

Given the model defined above, we need to identify the possible market-related variables, apart from the own lags of each component. In this study, we attempt to find variables with economic interpretation. In the literature, the most widely used variables have been spread, trading volume and price (see, for example, Hasbrouck (1996), Goodhart and O'Hara (1997), Coughenour and Shastri (1999) and Madhavan (2000)). The intuition is that trading activities and LOB trader behavior are related. For instance, in a volatile trading period, trading volume increases and 
trade duration and quote duration decrease. Consequently, these variations generate a volatile open LOB.

The first market-related variable is relative spread change, which is computed by the following formula:

RelativeSpread $_{k}=100 \cdot\left(\ln \left(a s k_{k}\right)-\ln \left(b_{i d}\right)\right)$.

Its variation is measured by:

DeltaSpread $_{k}=$ RelativeSpread $_{k}-$ RelativeSpread $_{k-1}$,

where ask and bid are the best sell price and best buy price available in open LOB. The advantage of relative spread is that it is dimensionless and can be used to directly compare different stocks. Given that the relative spread captures quasi-instantaneous information and might be noisy, another spread-related variable is the average relative spread over the ten most recent observations: AveSpread $_{k}=\frac{1}{10} \sum_{i=1}^{10}$ RelativeSpread $_{k-i}$.

Regarding the volume dimension, the first market-related variable is the square root of the volume (number of shares), SquareRoot (vol), that initiates the current trade. There are two reasons for the use of square root, one is to weigh down the large trade volume, and the second is that the price impact proves to be a concave function of market order size (Hasbrouck (1991)). If the volume that initiates the current trade is large, we expect a volatile situation and, consequently, the trade duration and quote duration are likely to be short.

The second volume-related variable should capture the imbalance of the signed trade. To this end, we adopt the depth measure proposed by Engle and Lange (2001), which is defined as follows:

$A b s(\text { sign.vol })_{k}=\mid \sum_{i=1}^{10} \operatorname{sign}_{k-i}$ volume $_{k-i} \mid$,

where the $\operatorname{sign}_{k-i}$ and $v_{\text {olume }} e_{-i}$ are the trade sign and trade volume for the $(k-i)$ th trade.

The trades are classified into buy-initiated and sell-initiated according to the rule of Lee and Ready (1991). Intuitively, when the depth measure increases, it indicates that the trades are imbalanced and the market is dominated by one-sided pressure.

The third dimension is duration. We define two sorts of duration, back-quote duration and quote-quote duration, which are different from trade duration and quote duration. The former is used to consider the duration between the first update of the LOB after the previous trade and the following trade, which contains quote information. Note that the back-quote duration could be zero due to the fact that the quote duration might be censored when the trade occurs before the update of open LOB. The way in which the data are sampled ignores some quotes when there is more than one update between two trades. This might not be a concern when 
$75 \%$ of the quotes are preserved, as in Engle and Lunde (2003). However, in a market where the open LOB is more active, as in the Xetra trading system, ignoring the quote activity may be a concern. In fact, only about $20 \%$ of the quotes are preserved in our dataset.

The quote-quote duration variable considers the duration for which there is no change in the EWS. As a result, it will be used only in explaining the components such as direction and size when the measure changes.

The above variables will be all (or partially) included in the market-related variable vectors for different components. In addition, we put time-of-day dummy variables in the models of the three factors to remove seasonality, a stylized fact in high-frequency data.

Table 1 presents the summary statistics of durations and market-related variables for May 2011. The number of monthly trade observations across all stocks ranges from 43,567 to 234,826 . The trade frequency is also confirmed by the corresponding average trade durations. That is, stocks with larger numbers of trades correspond to shorter trade durations. Regarding the average trade volumes, there are big differences: they vary from 180 to 1,669 Euros, meaning that the selected stocks have different trading volume patterns. The average quote durations are relatively small, and there is evidence that the dynamics in the open LOB are more active than those of trades. Considering other market-related variables, the averages of DeltaSpread and $\Delta E W S$ are naturally close to zero. AveSpread is dimensionless, so we can consider this variable as an indicator of the transaction costs for small volumes. The mean of AveSpread, for all stocks, is around $0.05 \%$, meaning that the average remains relatively stable across the stocks. The average trade imbalance variable Abs(sign.vol) varies from 767 to 20,726.9 Euros, indicating the existence of different trading patterns across the stocks.

[Insert Table 1 here]

\section{Estimation and Results on the EWS}

Our dataset covers three months: July 2010, May 2011 and June 2011. For the sake of brevity, we present and compare the estimation results for $\Delta E W S^{L o w}$ and $\Delta E W S^{H i g h}$ for May 2011. ${ }^{17}$ We estimate our model for the 30 stocks of the DAX using the first two-week data and do the out-of-sample test with the data of the third week. For each stock, $E W S^{\text {Low }}$ and $E W S^{\text {High }}$

\footnotetext{
${ }^{17}$ Results for the two other months are similar and available from the authors.
} 
represent ex-ante weighted spread based on the 20th and 80th percentiles of its own historical trading volume distribution.

\subsection{Temporal Factors}

The estimation results of deseasonalized trade and quote durations are presented in Table A.2 and Table A.4 of the appendix. Both tables show the estimated parameters for lagged autodependent and market-related variables. A more detailed analysis can be found in the online appendix.

\subsubsection{Trade Duration Factor}

In the modeling, the lagged auto-dependent variables are used to capture the degree of persistence in the trade durations. The market-related variables will capture the effect of different variables on trade durations.

The overall results on trade durations, presented in Table A.2, are stable across stocks over the three sample periods and provide new empirical evidence about trade duration dynamics. First, the sums of the coefficients for the auto-dependent part is around 0.9, suggesting that trade durations are highly persistent. Second, the coefficients for DeltaSpread and AveSpread are positive and significant for 30 and 25 stocks respectively, indicating that when liquidity decreases, traders slow down their trading intensities. Moreover, the coefficient for the short-term variable SquareRoot $(\mathrm{vol})$ is negative and significant for all stocks, indicating that large trades increase trading intensity. However, the coefficient for the long-term variable Abs(sign.vol) is positive and significant for 20 stocks. This means that, when the trade imbalance increases, trading activity slows down or keeps the same speed.

We validate our duration model by the Ljung-Box statistics. The results at different lags for the resulting standardized residuals are presented in Table A.3. Compared with the Ljung-Box statistics on trade durations (Table A.1), our results present evidence that the lagged autodependent part is capable of removing this autocorrelation feature in the trade durations because the Ljung-Box statistics have been reduced dramatically and, for most stocks, the hypothesis of no autocorrelation cannot be rejected. 


\subsubsection{Quote Duration Factor}

Similar to the trade duration equation, we include lagged auto-dependent structure and marketrelated variables as explanatory variables for the quote duration equation. We include more market-related variables in the quote duration equation than in the trade duration equation because we assume that the trade durations are market-related and can explain the quote duration dynamics. More specifically, the market-related variables we use to explain the dynamics of quote durations are: trade-duration-related variables, censored effect variable, DeltaSpread, AveSpread, SquareRoot(vol) and Abs(sign.vol). Table A.4 presents the corresponding estimate results.

The effect of market-related variables on the quote duration can be briefly summarized as follows: first, AveSpread and SquareRoot (vol) have a negative impact on quote durations. This suggests that when the average spread is large and the trading volume is high, LOB traders speed up their revisions. Second, the coefficient for the trade imbalance variable Abs(sign.vol) is significantly negative for the 30 stocks, suggesting that liquidity providers rapidly react to this imbalance. Comparing the Ljung-Box statistics on residuals from the quote duration model in Table A.5 with those of raw quote durations in Table A.1, we find that the statistics have been largely reduced and, for most stocks, the hypothesis of no autocorrelation cannot be rejected.

\subsection{Activity Factor}

Up to now, we have analyzed the dynamics of trade durations and quote durations. Conditional on the these temporal variables, we can further analyze other dimensions of weighted spread. As mentioned above, we decompose the change in the weighted spread into three components: Activity, Direction and Size. Similar to the time dimension, we also include lagged auto-dependent variables, market-related and time dummy variables in each factor modeling. The $M^{A}$ vector includes expected trade duration, expected quote duration, DeltaSpread, AveSpread, SquareRoot (vol), Abs(sign.vol), BackQuote duration, and $\triangle E W S$. We also include time dummy variables to control intraday seasonality.

Table 2 and Table A.7 report the estimated results of the weighted spread activity factor for $E W S^{H i g h}$ and $E W S^{L o w}$, respectively. The activity process is a binary process in which the value 1 means a change in the EWS. To capture the high persistence of autocorrelation in the 
activity factor, we adopt the GLARMA structure introduced by Rydberg and Shephard (2003). We also include market-related variables. As shown in Table 2, for all stocks, the coefficients of the "GLAR" part are positive and significant with a mean of 0.88 , which suggests high persistence in autocorrelation for the activity factor. More specifically, there is a cluster effect in activity: the change in the EWS is more likely to be followed by another change.

[Insert Table 2 here]

We are also interested in the effect of market-related variables on the dynamics of liquidity. For the time dimension variables, expected trade duration and expected quote duration do not have the same effect on the probability of EWS change. In particular, for the activity factor of $E W S^{H i g h}$, a longer expected trade duration increases the probability of EWS change significantly for 14 stocks, whereas a longer quote duration significantly decreases this probability for 29 stocks. The same effect of quote duration is found for the activity factor of $E W S^{L o w}$, and expected trade duration seems to have more impact on the activity factor of $E W S^{\text {Low }}$. In the tick-by-tick trading framework, as Dionne et al. (2009) and Dionne et al. (2015) demonstrate, a longer trade duration has a positive impact on price volatility. As a result, a longer trade duration increases the probability of EWS change. However, quote duration measures the quote intensity. A longer quote duration means a less active open LOB. Therefore, the quote is likely to be unchanged.

Regarding spread-related variables, AveSpread has a positive effect on the probability of EWS change for activity factor of $E W S^{H i g h}$ for all stocks, and the impact of DeltaSpread on the activity factor is negative. Because AveSpread measures relative long-term liquidity and DeltaSpread captures the dynamics of short-term liquidity, the estimation results suggest that the higherlevel of the LOB reacts more to relative long-term than short-term liquidity. When long-term liquidity decreases (higher AveSpread), LOB traders are more prudent, and are more likely to update their quotes. Therefore, the probability of a $E W S^{H i g h}$ change increases. Regarding the lower-level of the LOB, we find that the activity factor reacts both to DeltaSpread and AveSpread. Note that the activity factor only tells us whether the EWS changes or not; there is no information on the direction and magnitude of change.

Regarding volume-related variables, SquareRoot (vol) and Abs(sign.vol) affect the probability of $E W S^{\text {High }}$ change in different ways. The coefficients of SquareRoot (vol) are significantly positive for both $E W S^{L o w}$ and $E W S^{H i g h}$ changes. This means that short-term large trades 
are likely to increase the probability of EWS changes. As mentioned above, large trades are likely to be informative. Under this circumstance, LOB traders are more likely to review their quotes and then the resulting LOB changes. The effect of Abs(sign.vol) is not significant for the activity factor of $E W S^{H i g h}$ and significantly negative for that of $E W S^{L o w}$. This is not intuitive when we consider the trade imbalance as a measure of information asymmetry. One explanation is that algorithm traders stay at the lower-level of the LOB and provide liquidity by keeping a stable spread.

Another time dimension variable, BackQuote duration, also has a positive impact on the probability of weighted spread change. This is in line with the estimated results for trade duration. The effect of expected trade duration is higher than that of expected quote duration. Therefore, a longer BackQuote duration implies a more volatile market and the LOB is likely to be updated. For $E W S^{L o w}$ and $E W S^{H i g h}$, as expected, the coefficient is positive and significant at the $1 \%$ level for all stocks. This means that when a stock is less liquid or has more adverse selection risk, there is a greater chance that LOB traders will review their quote. The state of the LOB then changes. Concerning the time dummy variables (not presented), we find that there is a week seasonality pattern only for some stocks in certain time intervals; most of the periods do not exhibit a seasonality pattern.

We validate the activity factor modeling by several in-sample tests presented in Table 3 . The Ljung-Box statistics at different levels on the standardized residuals shows that the GLARMA part can capture this autocorrelation feature very well because all statistics have been reduced to the level of the critical values. Other in-sample tests presented in the same table includes the McFadden's pseudo-R squared, the Receiver Operating Characteristic (ROC) and Count accuracy. The average McFadden's pseudo-R squared is $23.93 \%$, average ROC is 0.612 and the count accuracy is $60.87 \%$.

[Insert Table 3 here]

\subsection{Direction Factor}

Direction is also a binary process: the value 1 means an increase in the EWS and -1 means a decrease in the EWS. Conditional on the activity factor, the direction factor gives more information about the change in the EWS. Table 4 presents the estimation results of the direction 
factor for the GLARMA structure and other market-related variables. Specifically, the sums of the "GLAR" part are around 0.6, suggesting a cluster effect, and the "MA" part is around -1.5, indicating a mean-reverting feature. In other words, the decrease in the EWS is likely to be followed by an increase in the EWS, and vice versa.

[Insert Table 4 here]

The market-related variables in the direction equation are: QuoteQuote duration, DeltaSpread, AveSpread, SquareRoot (vol), Abs(sign.vol), BackQuote duration and $\triangle E W S$. QuoteQuote duration is defined as the duration between two EWS changes. Given that the direction component is observed only when the activity factor is equal to one, it is more reasonable to use a temporal variable to capture this time interval. For the direction factor of $E W S^{L o w}$, we find that 26 stocks have a significant and negative coefficient for QuoteQuote duration. It appears that liquidity is likely to increase after a longer no-change period. However, the results for $E W S^{H i g h}$ shows that the higher-level of the LOB is less likely to be affected by the quoting intensity.

Considering the spread-related variables, a larger DeltaSpread is likely to cause a decrease in liquidity. Intuitively, when the spread increases, this means that LOB traders keep away from mid-quotes, so the EWS is likely to increase. The same results are found for the direction factor of $\Delta E W S^{\text {Low }}$. Compared with DeltaSpread, the AveSpread has the opposite effect on the direction factor. This suggests that the permanent increase in the spread is likely to lead to an increase in liquidity. It seems evident that when the traders have to pay a higher liquidity premium, the LOB traders (i.e, liquidity providers) are willing to provide liquidity by reducing the spread.

Regarding the volume-related variables, the coefficient of SquareRoot (vol) is positive for both $\Delta E W S^{H i g h}$ and $\Delta E W S^{L o w}$, meaning that the current large trades are likely to lead to a higher EWS, which is consistent with the literature. Intuitively, a large trade is likely to generate a higher price impact. However, the trade imbalance variable Abs(sign.vol) has a negative and significant effect on the direction factor for $E W S^{L o w}$ and confirms that when there is a need for liquidity, the liquidity providers increase liquidity by reducing spread. However, the effect of $A b s\left(\right.$ sign.vol) on the direction factor of $E W S^{H i g h}$ is much less pronounced. Only half of the stocks have a significantly negative effect, implying that the higher-level of the LOB is less sensitive to liquidity demand.

For $\Delta E W S^{L o w}$ and $\Delta E W S^{H i g h}$, an increase in EWS is likely to lead an increase in liquidity. 
This increase provides evidence of mean-reverting in EWS dynamics. We also use dummy variables to capture seasonality in the direction factor. The estimated results (not presented) show that the coefficients are not significant, meaning that there is no clear seasonality effect on the direction factor.

The in-sample test for the model of the direction factor are reported in Table 5 and Table A.10, respectively. The direction factor is highly autocorrelated and the hypothesis of no autocorrelation is rejected at all confidence levels for all stocks. However, the Ljung-Box statistics for the standardized residuals have been reduced significantly. We observe that the average McFadden's pseudo- $\mathrm{R}$ squared is $34.05 \%$, the average ROC is 0.797 and the count accuracy is $73.21 \%$.

[Insert Table 5 here]

\subsection{Size Factor}

The last factor is the size of the weighted spread change. Table 6 and Table A.11 report the estimated results for both $\Delta E W S^{\text {High }}$ and $\Delta E W S^{\text {Low }}$. The sums of the "GLAR" part are around 0.71, suggesting a cluster effect in the size factor.

[Insert Table 6 here]

We include the same market-related variables as in the equation of the direction factor. QuoteQuote duration has a significantly postive effect on most of stocks with respect to both $\Delta E W S^{\text {Low }}$ and $\Delta E W S^{H i g h}$. Recall that QuoteQuote duration is the duration between two EWS changes. It indicates that liquidity is likely to increase slightly after a longer no-change period. The temporal variable BackQuote duration has a positive and significant effect on $\lambda_{k}$ for both $\Delta E W S^{\text {Low }}$ and $\Delta E W S^{H i g h}$, indicating that even though the liquidity provider tries to incite the traders to trade by increasing the liquidity provision (our conclusion from the results of the direction equation), the magnitude of the liquidity increase (i.e., the EWS decreases) is moderate. However, if the trading intensity increases (i.e., the BackQuote duration decreases), liquidity is likely to decrease with a greater magnitude (i.e., EWS increases).

Regarding spread-related variables, a larger DeltaSpread is likely to cause a slight decrease in liquidity given that a higher DeltaSpread probably leads to a decrease in liquidity. Compared with DeltaSpread, AveSpread has the opposite effect on the size factor, which suggests that the 
permanent increase in the spread is likely to lead to a large increase in liquidity. It seems evident that when the traders have to pay a higher liquidity premium, LOB traders (i.e., liquidity providers) are willing to provide more liquidity by considerably reducing the spread. The coefficient of the SquareRoot $(v o l)$ is negative for both $\Delta E W S^{\text {High }}$ and $\Delta E W S^{\text {Low }}$ meaning that the current large trades are likely to lead to a much higher EWS. However, the trade imbalance variable Abs(sign.vol) has a significantly negative effect on the size factor for both $\Delta E W S^{H i g h}$ (18 stocks) and $\Delta E W S^{\text {Low }}(15$ stocks). One interpretation is that liquidity providers engage in aggressive spread reduction.

The last variable $\triangle E W S$ has a positive and significant effect on $\lambda_{k}$ for both $E W S^{L o w}$ and $E W S^{\text {High }}$ changes. This also suggests a slow resilience of the LOB. If the market is evaluated previously as less liquid based on EWS, the actual liquidity is prone to increase but the size of this increase is likely to be small.

We also attempt to capture seasonality by including time dummy variables in our model of size factor (not presented). Similar to the activity and direction factors, there is weak seasonality in the size component because most of the coefficients are not significant.

Table 7 reports the Ljung-Box statistics and adjusted $R^{2}$ of the size factor model. ${ }^{18}$ LjungBox statistics have been significantly reduced after the introduction of the GLARMA structure. However, we should reject the hypothesis that the series of standardized residuals is not autocorrelated. Therefore, the model might have a specification problem on $\theta_{k}^{S i z}$ or a mild distributional failure. Note that $\theta_{k}^{S i z}$ can be specified in many different ways and the distribution for size factor can differ from the geometric distribution. The average adjusted $R^{2}$ is $29 \%$.

[Insert Table 7 here]

\subsection{Summary of Market Related Variables' Impact}

We can compare the total effect of different market-related variables on changes in the Exante Weighted Spread (EWS). Table 8 summarizes the effect of key market-related variables on $\triangle E W S$. For both lower-level and higher level of the LOB, higher AveSpread predicts a lower EWS, suggesting mean-reversion dynamics in EWS. SquareRoot (vol) has a significant positive effect on all the stocks and predicts a large increase in EWS, confirming a rapid reaction of the

\footnotetext{
${ }^{18}$ Because the size factor is not binary variable, we present the Ljung-Box statistics and adjusted $R^{2}$ to validate the model.
} 
LOB to a large trade. Interestingly, the trade imbalance variable, Abs(sign.vol), affects LOB differently: for the lower-level of the LOB, $E W S^{L o w}$ is more likely to remain the same after a higher trade imbalance; and for the higher-level LOB, the dynamic of $E W S^{H i g h}$ is less sensitive to Abs(sign.vol). Finally, higher DeltaSpread is likely to lead a higher EWS for the lower-level of the LOB and have little impact on the higher-level of the LOB. Given these empirical results, we can conclude that 1) the LOB is constructed by active and sophisticated book traders, and they take positions in different levels of the LOB. 2) traders at the lower-level of the LOB are less worried about providing liquidity for a temporary liquidity shock proxied by order imbalance, and traders at the higher-level of the LOB are patient speculators who try to estimate the probability of large market order arrivals after a large transaction, as Rosu (2009) argued.

[Insert Table 8 here]

\subsection{Out-of-sample Performance}

Now, we turn our attention to the model's out-of-sample performance. Once the model is estimated, we follow Christoffersen (2003) and use Monte Carlo simulations to make multi-step forecasts and to test the model's performance. Parameters are estimated using data from the first two weeks of our sample, and the data from the third week are used to validate the model. Because the estimation is based on tick-by-tick frequency, one of the advantages is that one can compute the simulated liquidity for any interval without re-estimating the model. Given that the duration model is applied to deseasonalized duration, the simulated duration is not in calendar units. However, simulated duration and calendar time intervals are proportionally related. For instance, the same simulated interval relates to a shorter calendar time interval for a more liquid stock.

The simulations are realized as follows:

1. We generate the trading and quoting durations between two consecutive transactions and take them as input variables for other factors;

2. With the autoregressive structure, market condition variables and simulated durations in step 1), we obtain the corresponding activity, direction and size factors;

3. We repeat steps 1 and 2 for 5,000 paths. 
To evaluate the model's performance, we first conduct a zero-mean $t$-test for the simulated series, and then compare the unconditional distribution of the simulated series $\breve{Z}_{k}$ with that of the realized series, $Z_{k}$. Specifically, we compute the $p$-values for the one-sample zero mean $t$-test, the two-sample $t$-test and Pearson's Goodness-of-Fit test for each simulated series and take the average $p$-value. Table 9 presents the results of the corresponding statistical tests. It follows that 1) our model effectively captures the characteristics of unconditional distribution for higher-level changes, especially for the one-sample zero mean $t$-test and the two-sample $t$ test; 2) For lower-level changes, the model suffers a moderate loss of precision, suggesting some improvements could be gained from integrating more complicated distributions in our modeling.

[Insert Table 9 here]

In order to address the concern of how our model captures the temporal dimension of the real data, we also compare the sample autocorrelation function (ACF) of the simulated sample to that of the real data sample, and compute the mean squared error (MSE) of our simulated sample for various time horizons. We first compute the ACF for each simulated path of $\check{Z}_{k}$, and then take the average of the ACFs of the 5000 paths, and compare it with that of the real data. Table 10 reports the average simulated and empirical ACF from 1 to 5 lags. In general, we have negative ACFs for both simulated and empirical samples, which decrease as the lag increases. Also, the magnitude of our simulated ACFs is, on average, close to that of real data. Further, in Figure 4, we illustrate typical evolutions of $Z_{k}$ for our representative stocks EOAN and MAN. ${ }^{19}$ Lastly, we assess how the aggregated $\check{Z}_{k}$ from simulation deviates from real data by computing the aggregated $\breve{Z}_{k}$ for various tick lengths and comparing them with the corresponding aggregated $Z_{k}$ of the realized series. Table 11 shows the mean square error for various horizons of the 30 stocks. Our results show that our simulated data have small average errors for both short and long horizons.

\section{[Insert Table 10 here]}

\footnotetext{
${ }^{19}$ The variance of $E W S_{T}^{q}$ is equal to $T \times \sigma_{Z}^{2}\left(1+2 \sum_{t=1}^{T-1} A C F(t) \times(1-t / T)\right)$. It is a function of $T$ and $A C F(t)$. In our simulations, $E W S_{T}^{q}$ sometimes diverge gradually at large times even though $Z_{k}$ is stationary with zero mean and our model captures well the autocorrelation of empirical data. The variance of $E W S_{T}^{q}$ may become very large when the summation containing $A C F(t)$ does not efficiently cancel the effect of $T$. In practice, to avoid this blow-up at very large times, the aggregation of simulated $Z_{k}$ should be done with a given number of ticks. This is how we proceed when showing the economic significance of our model in Section 5. We thank a referee for pointing out this issue.
} 
[Insert Figure 4 here]

[Insert Table 11 here]

\section{Economic Value of the Model}

So far, our results provide statistically evidence in support of high persistence in autocorrelation and market condition variables. In this section, we show that the model we propose can also be economically significant. We do this by considering a simple stock liquidation scenario and comparing the trading costs between the uniform-order-submission strategy, the moving-averagesubmission strategy and the decomposition-model-based strategy. In real market, traders mainly have two sources of risk when liquidating a position: market risk and liquidity risk..$^{20}$ The order splitting practice can avoid the problem of price impact but extends order completion time, which makes market risk more pronounced, whereas large volume trades bear less market risk but have important price impacts. Given that our model focuses only on ex-ante liquidity (weighted spread), we set up the market environment as follows: 1) To ensure the same liquidation value for the three strategies, we require that the numbers of trades derived from the strategies are the same. In addition, for each trade, the volume is fixed. We choose the trade volume as the 20th percentile of historical trading volume distribution for each stock to have a small market impact; 2) all market-order submissions are in one-direction (buy only or sell only); 3) and, to have the minimum impact of market risk, we require model-based trades to follow the uniform trades closely. With these particular settings, our signals involve solely order submission time. Specifically, the trading strategy we consider can be summarized as follows: we first simulate 5,000 paths only with the GLAR part of the model. For each path, we simulate the same number of ticks as in real data. We use the GLAR part only in order to create a fair estimation scenario that does not contain information on the future market condition. Then, we aggregate the tickby-tick real and simulated series with a given number of ticks to form two samples. For each sample, we require our number of trades to equal $10 \%$ of total trades. The uniform strategy is applied directly to the real data sample and trade with a fixed frequency. For example, if we have 100 periods, the uniform strategy will trade 10 times at the 10th, 20th,..., and 100th time points,

\footnotetext{
${ }^{20}$ In this paper, market risk is related to uncertainty about stock mid-quote price, whereas liquidity risk is uncertainty about the shape of the LOB when liquidating the position.
} 
respectively. The moving-average-submission strategy generates signals based on the comparison between the actual spread and its historical average. ${ }^{21}$ More specifically, the moving-averagesubmission strategy trades when the actual spread is smaller than its historical average. This strategy continues until the required number of trades is obtained. For the 100-period scenario, we only trade the first ten times when the actual spread is smaller than its historical average. Finally, our model-based strategy uses multi-step forecasts derived from a simulated sample to generate trade signals. To see this, consider the same 100 periods. To generate the first trade signal, the model-based strategy compares the first ten simulated spreads and selects the period of the smallest one to send the first trade signal. Then, the model-based strategy repeats the same procedure for the second ten simulated spreads to generate the second trade signal. The model-based strategy ends up having the same number of trades (10) as the uniform and movingaverage strategies. By following the signals from the three liquidation strategies, we compute and compare the total spread paid by these strategies. The difference is the cost savings from the more effective strategy.

Table 12 compares the performance of these three strategies for aggregations of various ticks. The decomposition-model-based strategy significantly outperforms the uniform order-submission strategy for all aggregations. For example, for the period of 10-tick aggregation, without taking into account other trading costs such as fixed operation fee and expenses related to model implementation, the average cost savings from paying less spread is about $16.17 \%$. The economic gains decrease when competing with the moving-average-submission strategy. Based on the one-sample $t$-test which examines whether the model-based strategy dominates the uniform or moving average strategies, we conclude that on average, for aggregations of 30, 40, and 50 ticks, the decomposition-model-based strategy can always significantly generate cost savings. However, this cost-saving efficiency becomes less significant for aggregations of 10 and 20 ticks. ${ }^{22}$

[Insert Table 12 here]

\section{An Estimation of Resilience}

Another possible application of our model is to provide an estimation of market resilience based

\footnotetext{
${ }^{21}$ In this typical exercice, we use a moving window of 10 periods to calculate historical average.

${ }^{22}$ Consistent with many model forecasting studies, the results confirm that a simple moving-average strategy could be hard to beat when using real data.
} 
on estimated parameters. Resilience is difficult to observe and estimate in real time given that after one liquidity shock, there are often other transactions before liquidity reverts to its initial level. The estimation of resilience can be thought of as the impulse response function derived from the Vector Autoregressive model. In the previous section, we modeled the dynamics of expected spread changes and examine the impact of several market-related variables on the changes. In a general sense, trades are translated into a long-run effect on mid-quote and shortrun effect on spread. More specifically, the permanent price impact from trades will be reflected in a mid-quote increase or decrease over long periods. The spread around mid-quote, a proxy for liquidity, reverts to its initial state in the long-run if no other important liquidity shocks follow. This inherently dynamic feature of financial markets, well known as stock resilience, represents a vital dimension of market liquidity. There is time-based and probability-based resilience in the financial literature. Time-based resilience relates to the time required for the liquidity to revert to its initial state from a random, uninformative shock. In contrast, probability-based resilience refers to the probability that, after a liquidity shock, the spread reverts to its former level before the next transaction (Foucault et al. (2005)). In spite of its theoretical importance, stock resilience has received little attention in empirical research. With our decomposition model, we outline a framework based on Christoffersen (2012) and carry out a multi-step simulation experiment to deduce the time-based and probability-based resilience. In practice, our design allows practitioners to compare resiliency for different stocks and improve the efficiency of their optimal execution strategies.

As a simplified example, we suppose the following scenarios, (i) all market-related variables remain at their average levels, and (ii) there is an initial liquidity shock that is not followed by other significant liquidity shocks. The multi-step simulations are realized as follows:

1. We generate the duration between two consecutive transactions based on the estimated coefficients of the LogACD model;

2. With the simulated duration and estimated coefficients for the activity, direction and size factors, we generate $Z_{k}$ for the $n+1$ periods ahead ${ }^{23}$

3. Repeat steps 1 and 2 for 5,000 paths;

4. Calculate the average time (ticks) with which liquidity reverts to its initial state after a given liquidity shock.

\footnotetext{
${ }^{23} n$ is an arbitrary number of ticks and in our simulation, it takes the value of 1000.
} 
5. Count the number of paths where the spread reverts to its initial state for any given tick.

For comparison purposes, we further compute the empirical time-based and probability-based resilience for real data. Specifically, we take the mode of tick-by-tick spreads as our reference spread, and select the moments when the current spread is higher than the reference spread as the moments for the occurrence of a liquidity shock. Then, we classify liquidity shocks according to their magnitude and calculate reversion time for all shocks with the same magnitude. Therefore, for each magnitude of shock, we have a sample reversion time. The sample average is the time-based resilience, and the value of the empirical cumulative distribution function evaluated at a given time is the corresponding probability-based resilience. Table 13 presents simulated and empirical time-based resilience for different magnitudes of shocks. In general, the reversion time increases with the magnitude of the shock. Specifically, the average of empirical reversion time ranges from 11.7 ticks to 40.7 ticks for 1 and 5 BPS shocks, respectively. The corresponding simulated time-based resiliences are 12.6 and 42.8 , suggesting that our model produces comparable, but slightly longer, time-based resilience. Note that empirical resilience is also a noisy proxy of real resilience because other transactions often occur before liquidity reverts to its initial level. Table 14 reports probability-based resilience. The results are laid out similarly to those of time-based resilience. That is, the probability of recovery increases monotonically with the number of ticks and varies greatly across stocks. For one unit of liquidity shock, the empirical probability of recovery for a higher-level spread ranges from $49.79 \%$ on average at the 5th tick to $90.08 \%$ on average at the 30th tick. The corresponding simulated probability-based resilience is $48.98 \%$ and $73.76 \%$. A similar pattern of resilience is found for the lower-level LOB and other periods in our sample.

[Insert Table 13 here]

[Insert Table 14 here]

\section{Conclusion}

Since the introduction of the open LOB trading mechanism, the state of open LOB has received considerable attention from academics, practitioners, and regulators because of its importance 
in price formation and in gauging liquidity and information asymmetry. This paper focuses on a measure of the state of the LOB: the ex-ante weighted spread (EWS). Different from an expost measure of liquidity and information asymmetry, the EWS is an ex-ante volume-dependent measure. The computation of the measure requires information such as the prices and the corresponding quantities available in the open LOB.

To model the dynamics of the EWS, we adopt the decomposition approach proposed by Rogers and Zane (1998). The EWS changes have been decomposed into five factors: trade durations, quote durations, activity, direction and size. To investigate the dynamics of each component, we apply the relevant econometric models to each factor and include the lagged auto-dependent structure and a wide range of market-related variables in the models. The models are validated by several in-sample tests and out-of-sample tests.

The main empirical findings are as follows: By including different microstructure-based variables, we first find that most market-related variables can influence the dynamics of both trade and quote durations.Moreover, the quote durations are influenced by the dynamics of trade durations, market-related variables and $\triangle E W S$. Second, to model durations and $\triangle E W S$, it is essential to include the lagged auto-dependent structure to capture the high persistence of autocorrelation. Third, most market-related variables have significant impacts on the dynamics of EWS. Fourth, the lower- and higher-level LOB react to temporal spread and trade imbalance in different ways. Fifth, trade durations and quote durations have an obvious seasonality pattern, whereas the seasonality pattern for other factors is much weaker.

Having found statistically significant evidence in support of high persistence in autocorrelation and market condition variables, we also show, through a simple high frequency trading exercise, that the use of the model can also be economically important. Finally, we demonstrate how to use our model to estimate resilience that is difficult to observe in real time.

Future research can take several avenues. Our study focuses on the impact of market-related variables on EWS changes. One alternative is to investigate how EWS change co-moves with trades. Another research direction is to decompose the EWS changes in a different order or into different factors to answer other microstructure questions such as the effect of news on resilience. However, the unsynchronization of the trade durations and quote durations between different stocks is a challenge, which might require a more complicated econometric model and reasonable assumptions of dependence. 


\section{References}

Aitken, M., Comerton-Forde, C., 2003. How should liquidity be measured? Pacific-Basin Finance Journal 11, 45-59.

Amihud, Y., Mendelson, H., 1986. Asset pricing and the bid-ask spread. Journal of Financial Economics 17, $223-249$.

Anatolyev, S., Shakin, D., 2007. Trade intensity in the russian stock market: dynamics, distribution and determinants. Applied Financial Economics 17, 87-104.

Baruch, S., Panayides, M., Venkataraman, K., 2017. Informed trading and price discovery before corporate events. Journal of Financial Economics 125, 561-588.

Bauwens, L., Giot, P., 2000. The logarithmic ACD model: an application to the bid-ask quote process of three NYSE stocks. Annals of Economics and Statistics / Annales d'Économie et de Statistique, 117-149.

Beltran-Lopez, H., Giot, P., Grammig, J., 2009. Commonalities in the order book. Financial Markets and Portfolio Management 23, 209-242.

Beltran-Lopez, H., Grammig, J., Menkveld, A.J., 2011. Limit order books and trade informativeness. The European Journal of Finance , 1-23.

Bilodeau, Y., 2013. Xetraparser [computer software]. HEC Montréal .

Brogaard, J., Hagstromer, B., Norden, L., Riordan, R., 2015. Trading fast and slow: colocation and liquidity. Review of Financial Studies 28, 3407-3443.

Brogaard, J., Hendershott, T., Riordan, R., 2019. Price discovery without trading: Evidence from limit orders. The Journal of Finance.

Cao, C., Hansch, O., Wang, X., 2008. Order placement strategies in a pure limit order book market. Journal of Financial Research 31, 113-140.

Cenesizoglu., T., Dionne., G., Zhou, X., 2018. Asymmetric effects of the limit order book on price dynamics. Working Paper, Canada Research Chair in Risk Management .

Christoffersen, P., 2012. Elements of financial risk management, 2rd edition. Academic Press .

Coppejans, M., Domowitz, I., Madhavan, A., 2004. Resiliency in an automated auction. Working paper, ITG Group .

Coughenour, J., Shastri, K., 1999. Symposium on market microstructure: a review of empirical research. Financial Review 34, 1-27.

Cox, D.R., Gudmundsson, G., Lindgren, G., Bondesson, L., Harsaae, E., Laake, P., Juselius, K., Lauritzen, S.L., 1981. Statistical analysis of time series: some recent developments. Scandinavian Journal of Statistics 8, 93-115.

Dionne, G., Duchesne, P., Pacurar, M., 2009. Intraday value at risk (IVaR) using tick-by-tick data with application to the Toronto Stock Exchange. Journal of Empirical Finance 16, 777792 .

Dionne, G., Pacurar, M., Zhou, X., 2015. Liquidity-adjusted intraday value at risk modeling and risk management: An application to data from Deutsche Borse. Journal of Banking \& Finance 59, 202-219.

Domowitz, I., Hansch, O., Wang, X., 2005. Liquidity commonality and return co-movement. Journal of Financial Markets 8, 351-376. 
Easley, D., O'Hara, M., 1987. Price, trade size, and information in securities markets. Journal of Financial Economics 19, 69-90.

Engle, R.F., Lange, J., 2001. Predicting VNET: a model of the dynamics of market depth. Journal of Financial Markets 4, 113-142.

Engle, R.F., Lunde, A., 2003. Trades and quotes: a bivariate point process. Journal of Financial Econometrics 1, 159-188.

Engle, R.F., Russell, J.R., 1998. Autoregressive conditional duration: a new model for irregularly spaced transaction data. Econometrica 66, 1127-1162.

Foucault, T., 1999. Order flow composition and trading costs in a dynamic limit order market. Journal of Financial Markets 2, 99-134.

Foucault, T., Kadan, O., Kandel, E., 2005. Limit order book as a market for liquidity. The Review of Financial Studies 18, 1171-1217.

Giot, P., Grammig, J., 2006. How large is liquidity risk in an automated auction market? Empirical Economics 30, 867-887.

Glosten, L.R., 1994. Is the electronic open limit order book inevitable? The Journal of Finance $49,1127-1161$.

Glosten, L.R., Milgrom, P.R., 1985. Bid, ask and transaction prices in a specialist market with heterogeneously informed traders. Journal of Financial Economics 14, $71-100$.

Goettler, R., Parlour, C., Rajan, U., 2005. Equilibrium in a dynamic limit order market. The Journal of Finance 60, 2149-2192.

Goettler, R.L., Parlour, C.A., Rajan, U., 2009. Informed traders and limit order markets. Journal of Financial Economics 93, $67-87$.

Goodhart, C.A.E., O'Hara, M., 1997. High frequency data in financial markets: Issues and applications. Journal of Empirical Finance 4, 73-114.

Hagstromer, B., Norden, L., 2013. The diversity of high-frequency traders. Journal of Financial Markets 16, $741-770$.

Hasbrouck, J., 1991. Measuring the information content of stock trades. The Journal of Finance $46,179-207$.

Hasbrouck, J., 1996. Order characteristics and stock price evolution An application to program trading. Journal of Financial Economics 41, 129-149.

Hausman, J.A., Lo, A.W., MacKinlay, A.C., 1992. An ordered probit analysis of transaction stock prices. Journal of Financial Economics 31, 319-379.

Hendershott, T., Riordan, R., 2013. Algorithmic trading and the market for liquidity. Journal of Financial and Quantitative Analysis 48, 1001-1024.

Irvine, P.J., Benston, G.J., Kandel, E., 2000. Liquidity beyond the inside spread: measuring and using information in the limit order book. SSRN eLibrary .

Jain, P.K., 2005. Financial market design and the equity premium: electronic versus floor trading. The Journal of Finance 60, 2955-2985.

Kalay, A., Wohl, A., 2009. Detecting liquidity traders. The Journal of Financial and Quantitative Analysis 44, 29-54.

Kyle, A.S., 1985. Continuous auctions and insider trading. Econometrica 53, 1315-1335. 
Lee, C.M.C., Ready, M.J., 1991. Inferring trade direction from intraday data. The Journal of Finance 46, 733-746.

Lu, X., Abergel, F., 2018. High-dimensional hawkes processes for limit order books: modelling, empirical analysis and numerical calibration. Quantitative Finance 18, 249-264.

Madhavan, A., 2000. Market microstructure: a survey. Journal of Financial Markets 3, 205-258.

Manganelli, S., 2005. Duration, volume and volatility impact of trades. Journal of Financial Markets 8, 377-399.

McCulloch, R.E., Tsay, R.S., 2001. Nonlinearity in high-frequency financial data and hierarchical models. Studies in Nonlinear Dynamics \& Econometrics 5, 1-17.

Parlour, C., 1998. Price dynamics in limit order markets. Review of Financial Studies 11, 789.

Pascual, R., Veredas, D., 2010. Does the open limit order book matter in explaining informational volatility? Journal of Financial Econometrics 8, 57-87.

Riordan, R., Storkenmaier, A., 2012. Latency, liquidity and price discovery. Journal of Financial Markets 15, $416-437$.

Rock, K., 1990. The specialist's order book and price anomalies. Working Paper,Harvard University .

Rogers, L.C.G., Zane, O., 1998. Designing and estimating models of high-frequency data. Unpublished paper .

Rosu, I., 2009. A dynamic model of the limit order book. The Review of Financial Studies 22, $4601-4641$

Russell, J., Engle, R., 2005. A discrete-state continuous-time model of financial transactions prices and times: the autoregressive conditional multinomial-autoregressive conditional duration model. Journal of Business \& Economic Statistics 23, 166.

Rydberg, T.H., Shephard, N., 2003. Dynamics of trade-by-trade price movements: decomposition and models. Journal of Financial Econometrics 1, 2-25.

Shephard, N., 1995. Generalized linear autoregressions. Working paper, University of Oxford .

Swets, J.A., 1986. Indices of discrimination or diagnostic accuracy: Their rocs and implied models. Psychological Bulletin 99(1), 100-117.

Swets, J.A., 1988. Measuring the accuracy of diagnostic systems. Science 240, 1285-1293. 
Figure 1: Snapshot of Supply and Demand of Liquidity in the LOB

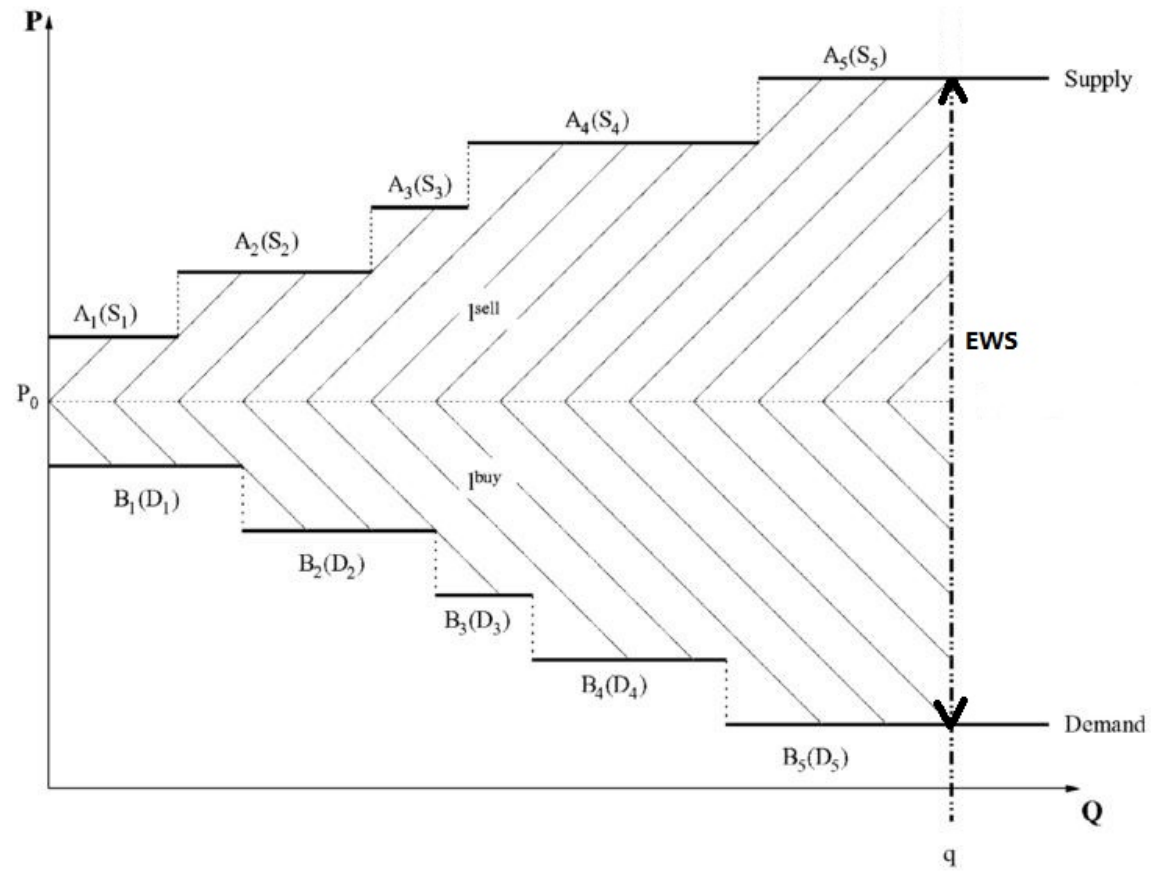

This figure presents a snapshot of the LOB and a relationship between EWS and a potential trade volume $q$. $P_{0}$ is the mid-quote price and $A_{i}\left(S_{i}\right)\left(B_{i}\left(D_{i}\right)\right)$ relates to Ask-side Supply (Bid-side Demand) at the level $i$. 


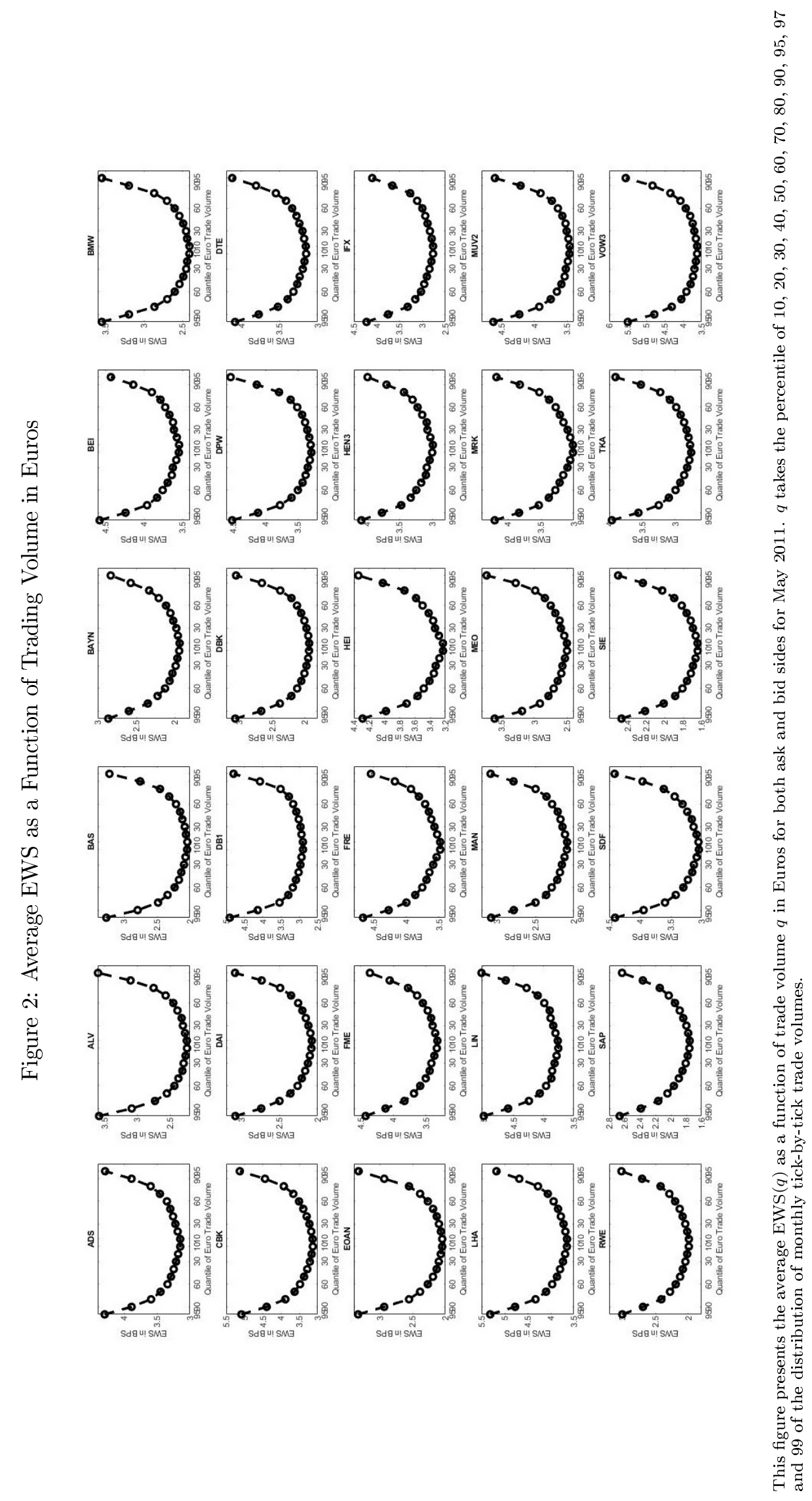


Figure 3: Timestamps for Trades and Quote Update in Open LOB

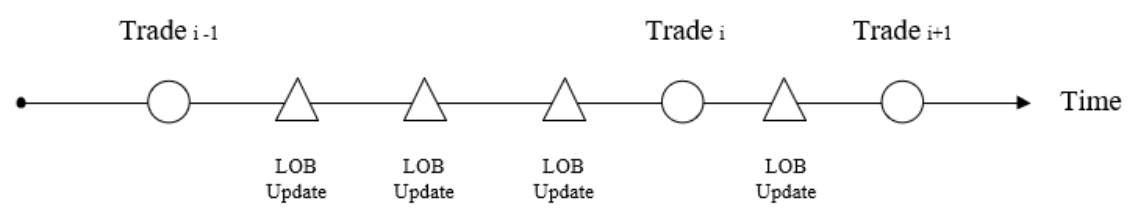

This figure presents the temporal relation between trades and quote updates, which are represented by circles and triangles, respectively. 

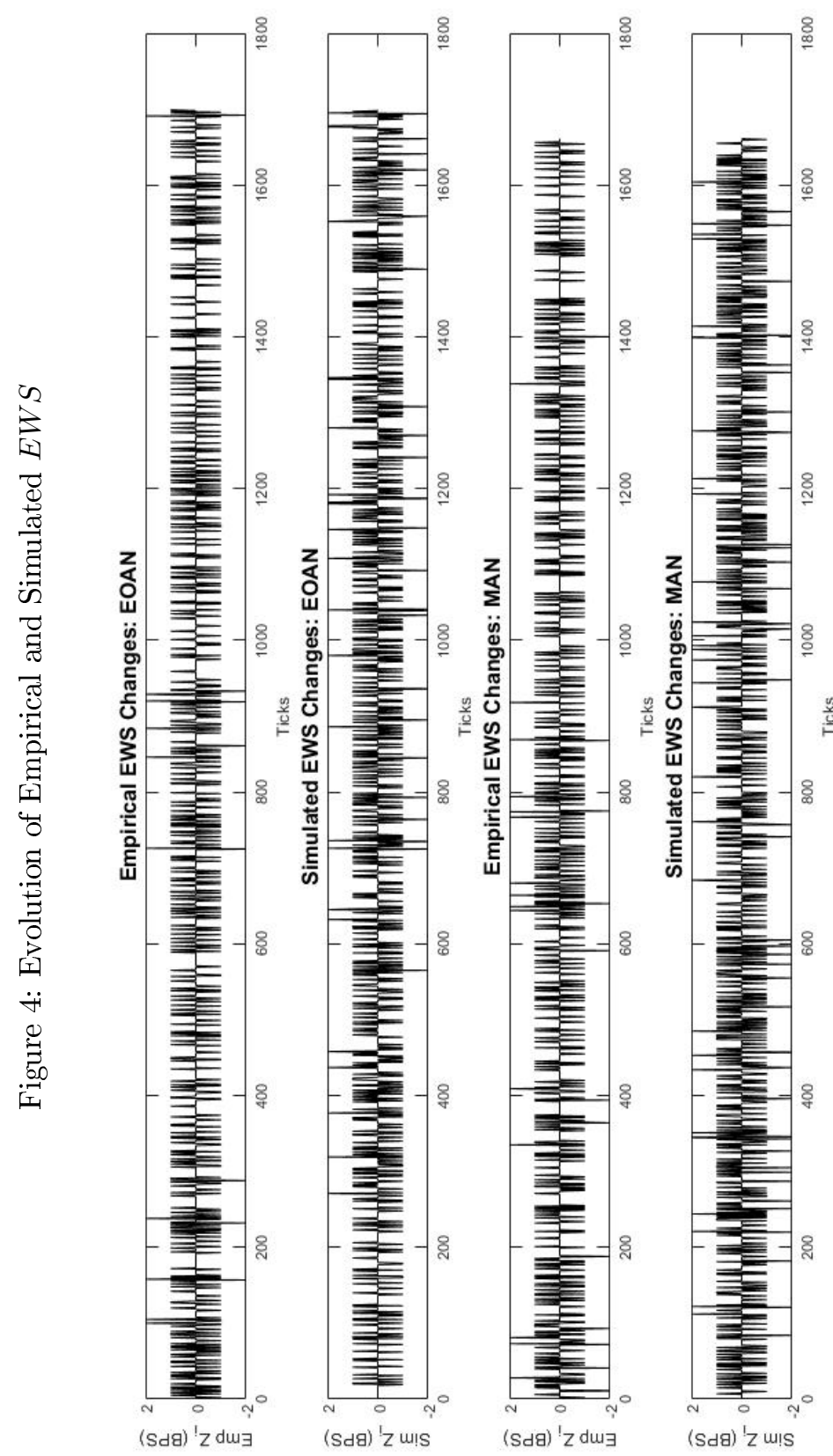


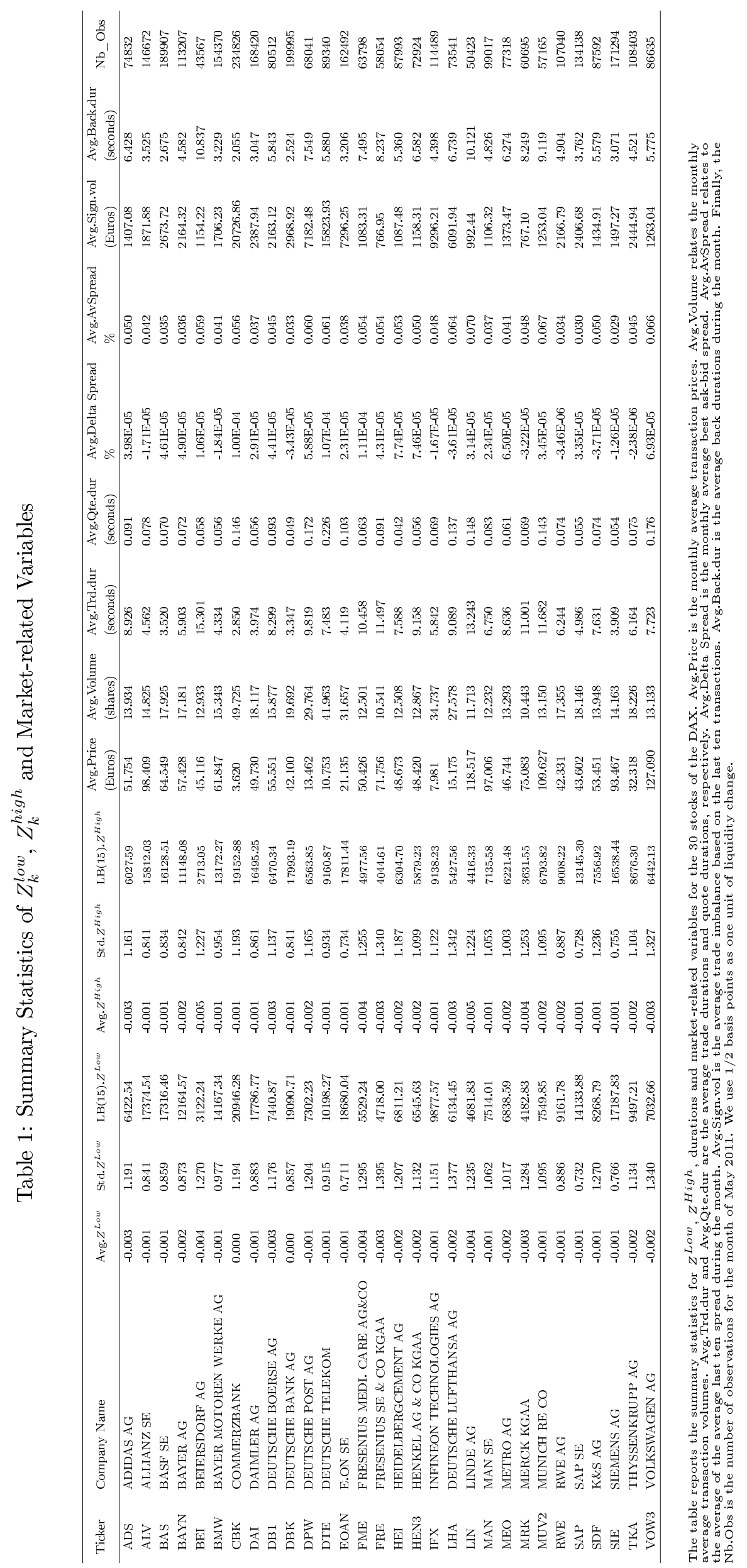




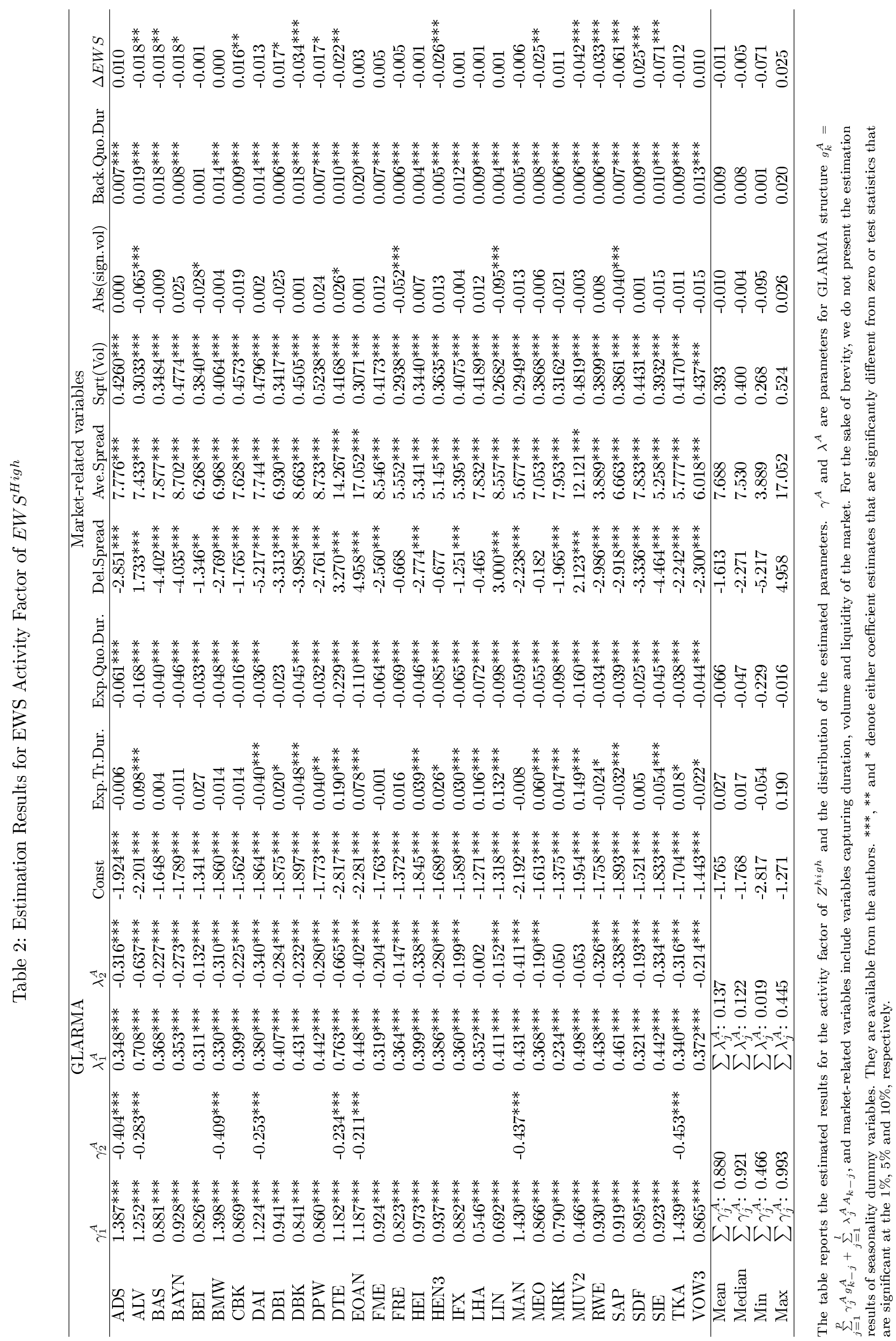


Table 3: In-sample tests for EWS Activity Factor of $E W S^{H i g h}$

\begin{tabular}{lllllll}
\hline Ticker & LB(5) & LB(10) & LB(15) & R-sqrt & ROC & \#_Acc \\
\hline ADS & 2.219 & 18.049 & 20.659 & $19.78 \%$ & 0.615 & $58.73 \%$ \\
ALV & 23.910 & 34.506 & 38.613 & $34.15 \%$ & 0.660 & $65.06 \%$ \\
BAS & 14.154 & 33.652 & 38.929 & $32.10 \%$ & 0.587 & $63.53 \%$ \\
BAYN & 10.666 & 22.667 & 24.255 & $30.38 \%$ & 0.599 & $62.57 \%$ \\
BEI & 5.411 & 10.839 & 11.966 & $14.82 \%$ & 0.590 & $57.70 \%$ \\
BMW & 11.554 & 17.168 & 18.734 & $24.62 \%$ & 0.594 & $59.29 \%$ \\
CBK & 19.949 & 33.050 & 38.978 & $18.50 \%$ & 0.614 & $58.74 \%$ \\
DAI & 7.139 & 14.472 & 32.525 & $27.86 \%$ & 0.606 & $61.62 \%$ \\
DB1 & 20.473 & 29.478 & 34.399 & $23.36 \%$ & 0.622 & $60.08 \%$ \\
DBK & 31.151 & 44.858 & 50.491 & $33.94 \%$ & 0.612 & $65.02 \%$ \\
DPW & 4.399 & 9.066 & 12.763 & $18.57 \%$ & 0.624 & $59.53 \%$ \\
DTE & 29.413 & 33.743 & 40.079 & $34.93 \%$ & 0.687 & $65.59 \%$ \\
EOAN & 16.660 & 25.716 & 33.443 & $35.16 \%$ & 0.630 & $65.50 \%$ \\
FME & 17.637 & 27.650 & 30.790 & $16.99 \%$ & 0.629 & $59.98 \%$ \\
FRE & 2.116 & 8.552 & 11.042 & $16.12 \%$ & 0.605 & $58.41 \%$ \\
HEI & 41.532 & 47.404 & 47.918 & $18.84 \%$ & 0.612 & $59.04 \%$ \\
HEN3 & 25.981 & 34.059 & 41.970 & $21.50 \%$ & 0.610 & $59.01 \%$ \\
IFX & 15.971 & 22.613 & 30.628 & $20.74 \%$ & 0.602 & $58.15 \%$ \\
LHA & 1.313 & 5.738 & 17.694 & $14.31 \%$ & 0.605 & $58.72 \%$ \\
LIN & 3.200 & 6.844 & 17.650 & $14.57 \%$ & 0.603 & $58.99 \%$ \\
MAN & 11.935 & 33.929 & 43.124 & $27.94 \%$ & 0.630 & $62.11 \%$ \\
MEO & 13.448 & 24.031 & 30.312 & $24.36 \%$ & 0.605 & $59.42 \%$ \\
MRK & 7.958 & 16.057 & 18.399 & $18.64 \%$ & 0.596 & $57.46 \%$ \\
MUV2 & 3.545 & 8.215 & 11.460 & $18.98 \%$ & 0.627 & $59.54 \%$ \\
RWE & 21.055 & 29.173 & 34.746 & $30.86 \%$ & 0.597 & $62.97 \%$ \\
SAP & 28.433 & 35.577 & 38.050 & $36.15 \%$ & 0.598 & $66.49 \%$ \\
SDF & 11.341 & 15.949 & 27.857 & $17.48 \%$ & 0.608 & $58.57 \%$ \\
SIE & 26.444 & 34.276 & 44.310 & $35.94 \%$ & 0.596 & $66.29 \%$ \\
TKA & 5.540 & 7.880 & 10.932 & $22.79 \%$ & 0.604 & $58.95 \%$ \\
VOW3 & 17.429 & 20.236 & 30.089 & $13.61 \%$ & 0.603 & $58.99 \%$ \\
\hline Mean & 15.066 & 23.515 & 29.427 & $23.93 \%$ & 0.612 & $60.87 \%$ \\
Median & 13.801 & 23.349 & 30.709 & $22.15 \%$ & 0.606 & $59.47 \%$ \\
Min & 1.313 & 5.738 & 10.932 & $13.61 \%$ & 0.587 & $57.46 \%$ \\
Max & 41.532 & 47.404 & 50.491 & $36.15 \%$ & 0.687 & $66.49 \%$ \\
\hline & & & & & &
\end{tabular}

The table reports the results of in-sample tests for the model of the activity factor. $\operatorname{LB}(5), \operatorname{LB}(10)$ and $\operatorname{LB}(15)$ are Ljung-Box statistics on 5, 10 and 15 lagged standardized residuals. The critical value for $\mathrm{LB}(5), \mathrm{LB}(10)$, and $\mathrm{LB}(15)$, are 11.07, 18.30 and 24.99, respectively. R-sqrt is the McFadden's R squared, defined as $R_{M c F a d e n}^{2}=1-\frac{\log \left(L_{c}\right)}{\log \left(L_{n u l l}\right)}$ where $L_{c}$ denotes the likelihood value from the current fitted model and $L_{\text {null }}$ denotes the corresponding value for the null model. ROC relates the Receiver Operating Characteristic test. \#_Acc is the Count accuracy that takes $50 \%$ as the threshold to have value one. 


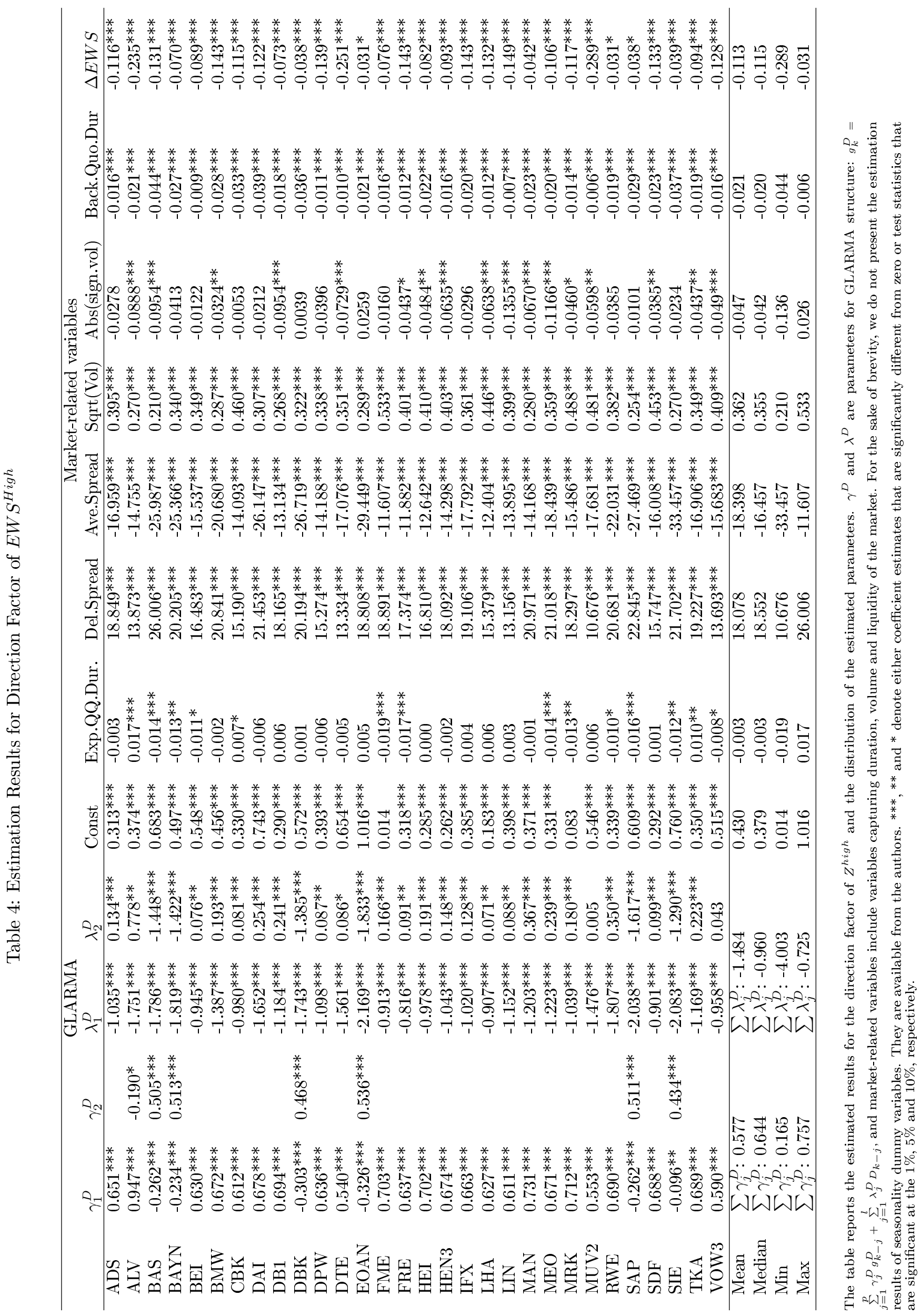


Table 5: In-sample tests for EWS Direction Factor of $E W S^{\text {High }}$

\begin{tabular}{|c|c|c|c|c|c|c|}
\hline Ticker & $\mathrm{LB}(5)$ & $\mathrm{LB}(10)$ & $\mathrm{LB}(15)$ & R-sqrt & $\mathrm{ROC}$ & \#_Acc \\
\hline ADS & 16.754 & 19.629 & 26.579 & $30.20 \%$ & 0.770 & $70.65 \%$ \\
\hline $\mathrm{ALV}$ & 9.259 & 24.103 & 31.341 & $41.14 \%$ & 0.844 & $78.05 \%$ \\
\hline BAS & 30.498 & 45.739 & 63.983 & $39.66 \%$ & 0.841 & $76.88 \%$ \\
\hline BAYN & 19.822 & 25.668 & 29.043 & $40.42 \%$ & 0.845 & $77.41 \%$ \\
\hline BEI & 19.277 & 22.232 & 27.909 & $28.30 \%$ & 0.754 & $69.05 \%$ \\
\hline BMW & 21.935 & 31.971 & 35.402 & $34.46 \%$ & 0.804 & $73.80 \%$ \\
\hline CBK & 9.092 & 17.837 & 21.840 & $29.10 \%$ & 0.759 & $69.67 \%$ \\
\hline DAI & 11.084 & 20.910 & 27.211 & $38.34 \%$ & 0.831 & $76.05 \%$ \\
\hline DB1 & 3.546 & 17.957 & 23.505 & $30.68 \%$ & 0.778 & $71.34 \%$ \\
\hline DBK & 21.048 & 35.665 & 43.608 & $38.82 \%$ & 0.835 & $76.58 \%$ \\
\hline DPW & 2.831 & 21.182 & 28.870 & $31.58 \%$ & 0.779 & $71.32 \%$ \\
\hline DTE & 9.281 & 12.901 & 14.393 & $39.02 \%$ & 0.833 & $76.93 \%$ \\
\hline EOAN & 43.588 & 46.919 & 52.397 & $48.66 \%$ & 0.882 & $81.79 \%$ \\
\hline FME & 16.364 & 24.499 & 29.680 & $28.80 \%$ & 0.758 & $69.61 \%$ \\
\hline FRE & 1.820 & 14.510 & 21.628 & $27.31 \%$ & 0.751 & $69.12 \%$ \\
\hline HEI & 13.450 & 18.759 & 25.374 & $28.34 \%$ & 0.758 & $69.81 \%$ \\
\hline HEN 3 & 13.724 & 16.294 & 19.716 & $29.23 \%$ & 0.764 & $70.14 \%$ \\
\hline IFX & 4.792 & 7.171 & 13.140 & $30.65 \%$ & 0.771 & $70.55 \%$ \\
\hline LHA & 14.649 & 17.871 & 23.206 & $28.81 \%$ & 0.758 & $69.21 \%$ \\
\hline LIN & 12.634 & 19.696 & 29.718 & $31.71 \%$ & 0.782 & $71.56 \%$ \\
\hline MAN & 11.587 & 21.958 & 38.927 & $30.38 \%$ & 0.777 & $71.32 \%$ \\
\hline $\mathrm{MEO}$ & 2.430 & 18.065 & 23.143 & $31.79 \%$ & 0.787 & $71.91 \%$ \\
\hline MRK & 5.961 & 9.387 & 18.079 & $30.33 \%$ & 0.771 & $70.81 \%$ \\
\hline MUV2 & 4.066 & 14.645 & 17.324 & $39.25 \%$ & 0.831 & $76.81 \%$ \\
\hline RWE & 7.134 & 13.275 & 18.429 & $39.18 \%$ & 0.837 & $76.87 \%$ \\
\hline SAP & 22.690 & 35.837 & 47.941 & $43.09 \%$ & 0.861 & $79.22 \%$ \\
\hline $\mathrm{SDF}$ & 4.687 & 9.073 & 11.193 & $28.84 \%$ & 0.760 & $69.61 \%$ \\
\hline SIE & 35.774 & 41.509 & 45.035 & $43.37 \%$ & 0.862 & $79.12 \%$ \\
\hline TKA & 9.923 & 15.957 & 19.692 & $31.17 \%$ & 0.779 & $71.56 \%$ \\
\hline VOW3 & 10.233 & 24.396 & 39.770 & $28.87 \%$ & 0.761 & $69.48 \%$ \\
\hline Mean & 13.664 & 22.187 & 28.936 & $34.05 \%$ & 0.797 & $73.21 \%$ \\
\hline Median & 11.335 & 19.662 & 26.895 & $31.37 \%$ & 0.779 & $71.45 \%$ \\
\hline Min & 1.820 & 7.171 & 11.193 & $27.31 \%$ & 0.751 & $69.05 \%$ \\
\hline Max & 43.588 & 46.919 & 63.983 & $48.66 \%$ & 0.882 & $81.79 \%$ \\
\hline
\end{tabular}

The table reports the results of in-sample tests for the model of the direction factor. $\operatorname{LB}(5), \operatorname{LB}(10)$ and $\operatorname{LB}(15)$ are Ljung-Box statistics on 5,10 and 15 lagged standardized residuals. The critical value for $\operatorname{LB}(5), \operatorname{LB}(10), \operatorname{LB}(15)$, are 11.07, 18.30 and 24.99, respectively. R-sqrt is the McFadden's R squared, defined as $R_{M c F a d e n}^{2}=1-\frac{\log \left(L_{c}\right)}{\log \left(L_{n u l l}\right)}$ where $L_{c}$ denotes the likelihood value from the current fitted model and $L_{n u l l}$ denotes the corresponding value for the null model. ROC relates the Receiver Operating Characteristic test. \#_Acc is the Count accuracy that takes $50 \%$ as the threshold to have value one. 


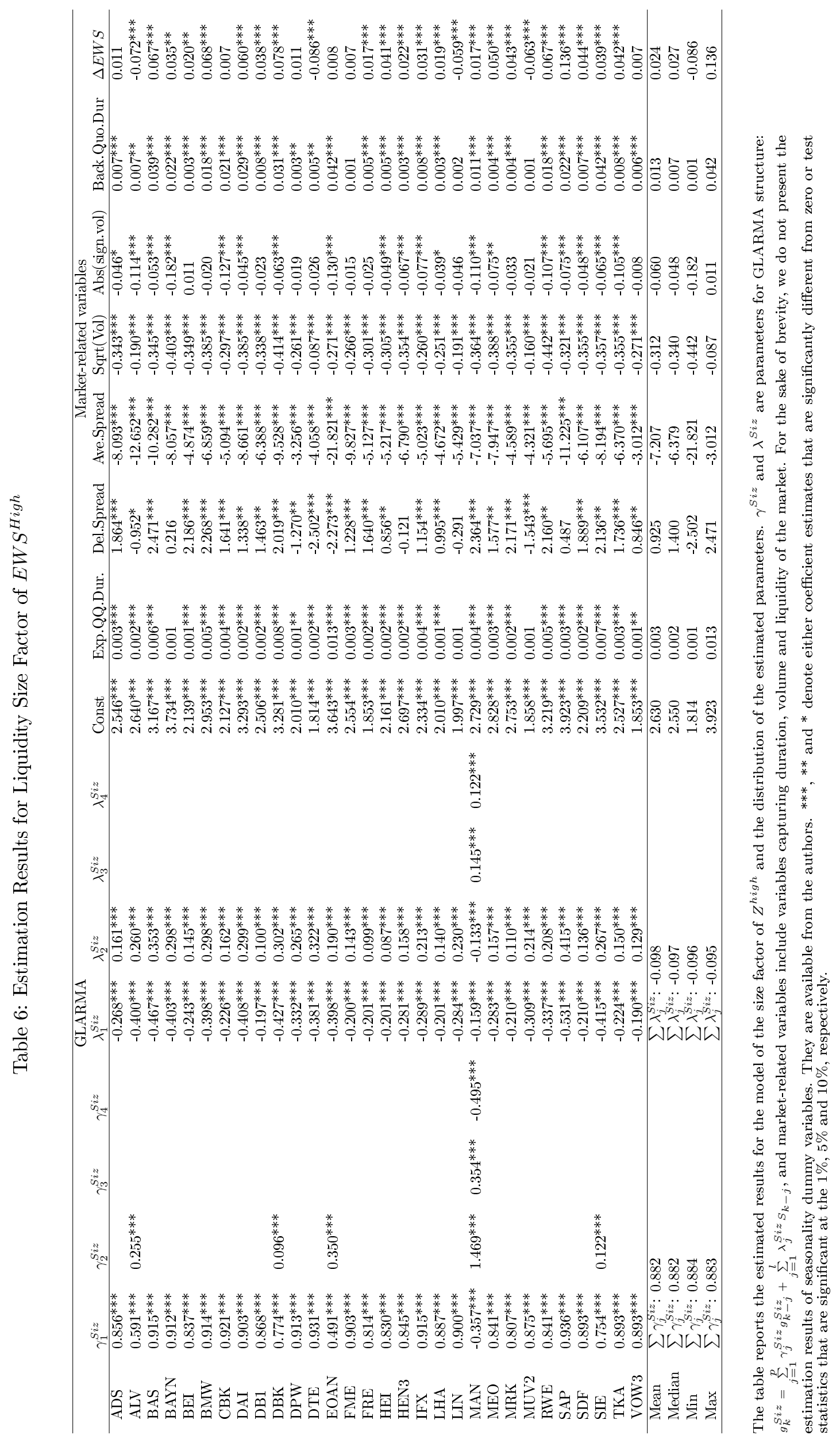


Table 7: Ljung-Box Statistics for EWS Size Factor of $E W S^{H i g h}$

\begin{tabular}{lllll}
\hline Ticker & LB(5) & LB(10) & LB(15) & R-sqrt \\
\hline ADS & 95.70 & 108.80 & 130.70 & $33.73 \%$ \\
ALV & 287.19 & 305.70 & 315.16 & $6.41 \%$ \\
BAS & 221.41 & 260.39 & 309.56 & $6.77 \%$ \\
BAYN & 176.57 & 200.02 & 211.18 & $3.21 \%$ \\
BEI & 65.05 & 70.04 & 87.39 & $44.55 \%$ \\
BMW & 222.37 & 244.43 & 274.53 & $12.69 \%$ \\
CBK & 106.83 & 124.26 & 135.49 & $33.76 \%$ \\
DAI & 177.17 & 195.01 & 207.04 & $9.40 \%$ \\
DB1 & 209.51 & 226.88 & 248.07 & $18.36 \%$ \\
DBK & 326.86 & 350.42 & 386.83 & $48.26 \%$ \\
DPW & 37.58 & 41.47 & 44.18 & $37.99 \%$ \\
DTE & 185.71 & 188.59 & 192.32 & $37.26 \%$ \\
EOAN & 343.36 & 398.26 & 493.74 & $26.25 \%$ \\
FME & 153.91 & 189.04 & 196.94 & $34.72 \%$ \\
FRE & 44.18 & 47.81 & 61.49 & $47.51 \%$ \\
HEI & 180.14 & 204.21 & 233.65 & $20.43 \%$ \\
HEN3 & 158.55 & 171.83 & 176.75 & $6.54 \%$ \\
IFX & 108.84 & 113.37 & 128.35 & $31.57 \%$ \\
LHA & 73.14 & 79.96 & 101.33 & $40.91 \%$ \\
LIN & 43.30 & 45.34 & 52.43 & $39.20 \%$ \\
MAN & 421.59 & 539.34 & 635.15 & $20.27 \%$ \\
MEO & 86.72 & 123.41 & 140.14 & $40.63 \%$ \\
MRK & 145.52 & 169.79 & 181.95 & $66.15 \%$ \\
MUV2 & 167.49 & 172.99 & 179.35 & $42.68 \%$ \\
RWE & 242.26 & 269.68 & 288.75 & $12.41 \%$ \\
SAP & 191.95 & 217.20 & 233.29 & $30.90 \%$ \\
SDF & 143.78 & 163.63 & 175.70 & $34.37 \%$ \\
SIE & 416.45 & 474.87 & 515.65 & $6.31 \%$ \\
TKA & 248.86 & 279.60 & 298.74 & $22.68 \%$ \\
VOW3 & 82.97 & 87.87 & 107.47 & $50.63 \%$ \\
\hline Mean & 178.83 & 202.14 & 224.78 & $28.88 \%$ \\
Median & 172.03 & 188.82 & 194.63 & $32.65 \%$ \\
Min & 37.58 & 41.47 & 44.18 & $3.21 \%$ \\
Max & 421.59 & 539.34 & 635.15 & $66.15 \%$ \\
\hline & & & &
\end{tabular}

The table reports the results of Ljung-Box statistics on 5, 10 and 15 lagged standardized residuals and adjusted $R^{2}$. The critical value for $\mathrm{LB}(5), \mathrm{LB}(10)$, and $\mathrm{LB}(15)$, are $11.07,18.30$ and 24.99 , respectively. 


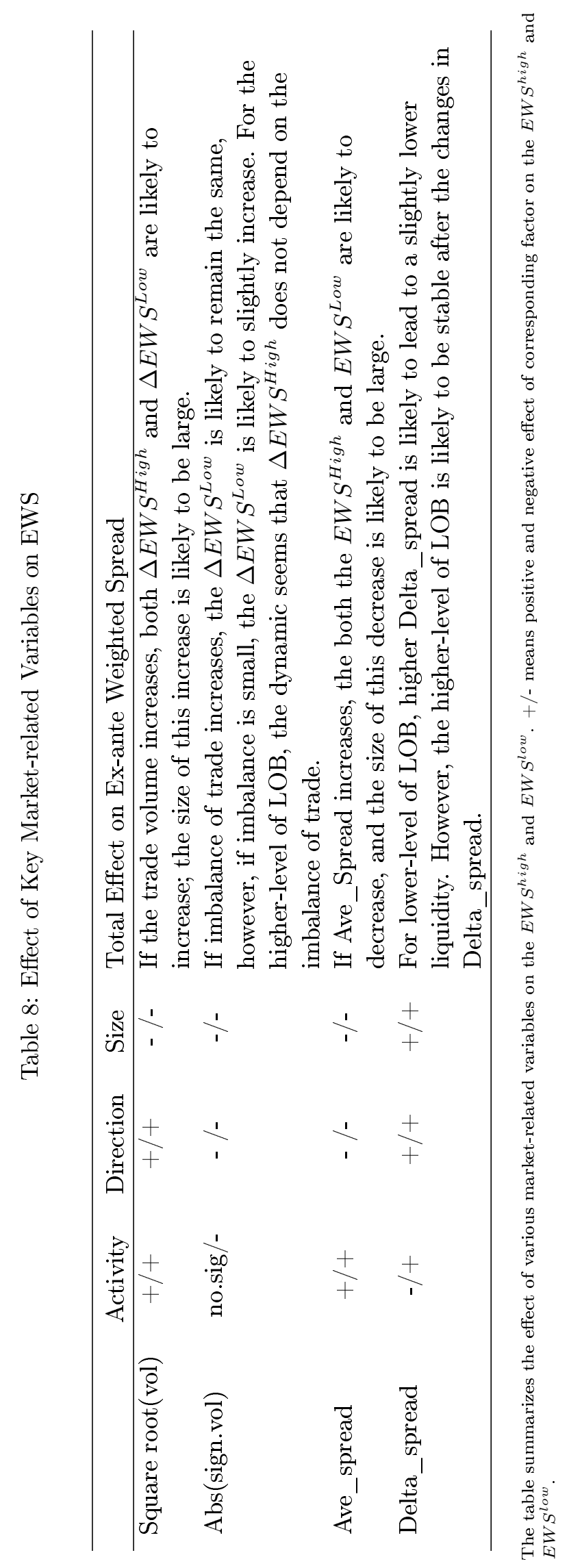


Table 9: Out-of-sample Test for Unconditional Distribution

\begin{tabular}{|c|c|c|c|c|c|c|}
\hline & \multirow{2}{*}{$\begin{array}{l}\check{Z}^{H i g h}=0 \\
p \text {-value0 }\end{array}$} & \multicolumn{2}{|c|}{$\check{Z}^{H i g h}=Z^{H i g h}$} & \multirow{2}{*}{$\begin{array}{l}\check{Z}^{\text {Low }}=0 \\
p \text {-value0 }\end{array}$} & \multicolumn{2}{|c|}{$\check{Z}^{\text {Low }}=Z^{\text {Low }}$} \\
\hline & & $p$-value1 & $p$-value2 & & $p$-value1 & $p$-value 2 \\
\hline ADS & 0.685 & 0.703 & $<0.001$ & 0.069 & 0.169 & 0.048 \\
\hline ALV & $<0.001$ & 0.001 & 0.224 & 0.109 & 0.197 & 0.282 \\
\hline BAS & 0.374 & 0.641 & 0.308 & 0.606 & 0.652 & 0.439 \\
\hline BAYN & 0.671 & 0.836 & 0.041 & 0.246 & 0.452 & 0.131 \\
\hline BEI & 0.214 & 0.115 & 0.002 & 0.055 & 0.064 & 0.006 \\
\hline BMW & 0.539 & 0.769 & 0.573 & 0.578 & 0.609 & 0.452 \\
\hline CBK & 0.035 & 0.140 & 0.128 & 0.003 & 0.013 & $<0.001$ \\
\hline DAI & 0.244 & 0.351 & 0.296 & 0.092 & 0.134 & 0.015 \\
\hline DB1 & 0.382 & 0.692 & 0.457 & 0.288 & 0.316 & 0.007 \\
\hline DBK & 0.561 & 0.662 & $<0.001$ & 0.371 & 0.493 & 0.046 \\
\hline DPW & 0.684 & 0.773 & 0.492 & 0.476 & 0.568 & 0.061 \\
\hline DTE & 0.713 & 0.728 & 0.617 & 0.011 & 0.044 & $<0.001$ \\
\hline EOAN & 0.557 & 0.693 & 0.042 & 0.182 & 0.208 & 0.020 \\
\hline FME & 0.091 & 0.127 & 0.151 & $<0.001$ & $<0.001$ & $<0.001$ \\
\hline FRE & 0.003 & 0.031 & $<0.001$ & $<0.001$ & 0.007 & $<0.001$ \\
\hline HEI & 0.011 & 0.041 & 0.008 & 0.003 & 0.005 & $<0.001$ \\
\hline HEN3 & 0.452 & 0.406 & 0.182 & 0.001 & 0.018 & 0.021 \\
\hline IF X & 0.638 & 0.722 & 0.351 & $<0.001$ & 0.030 & 0.001 \\
\hline LHA & 0.261 & 0.602 & 0.171 & 0.633 & 0.740 & 0.009 \\
\hline LIN & 0.578 & 0.768 & 0.246 & 0.594 & 0.571 & 0.387 \\
\hline MAN & 0.038 & 0.056 & $<0.001$ & 0.002 & 0.004 & $<0.001$ \\
\hline MEO & 0.193 & 0.214 & 0.164 & 0.010 & 0.022 & $<0.001$ \\
\hline MRK & 0.280 & 0.363 & 0.092 & 0.006 & 0.062 & $<\mathbf{0 . 0 0 1}$ \\
\hline MUV2 & 0.332 & 0.620 & $<0.001$ & 0.392 & 0.602 & 0.176 \\
\hline RWE & 0.719 & 0.731 & 0.063 & 0.605 & 0.628 & 0.023 \\
\hline SAP & 0.565 & 0.524 & 0.051 & 0.546 & 0.538 & 0.038 \\
\hline SDF & 0.670 & 0.783 & 0.072 & 0.335 & 0.454 & 0.392 \\
\hline SIE & 0.567 & 0.601 & $<0.001$ & 0.389 & 0.447 & 0.021 \\
\hline TKA & 0.700 & 0.786 & 0.391 & 0.228 & 0.243 & 0.075 \\
\hline VOW3 & 0.446 & 0.758 & 0.376 & 0.197 & 0.563 & 0.134 \\
\hline Mean & 0.508 & 0.508 & 0.183 & 0.234 & 0.295 & 0.093 \\
\hline Median & 0.631 & 0.631 & 0.139 & 0.190 & 0.226 & 0.021 \\
\hline Min & $<0.001$ & 0.001 & $<0.001$ & $<0.001$ & $<0.001$ & $<0.001$ \\
\hline Max & 0.836 & 0.836 & 0.617 & 0.633 & 0.740 & 0.452 \\
\hline
\end{tabular}

The table presents the $p$-values of the one-sample $t$-test ( $p$-value0), two-sample $t$-test ( $p$-value1) and Pearson's chi-square goodness-of-fit test ( $p$-value2) between the real time series and the simulated one for 30 stocks in DAX30 in the third week of May 2011. The null hypothesis for the one-sample $t$-test is that the means of the sample is equal to zero. The null hypothesis for the two-sample $t$-test is that the means of the two samples are equal. The null hypothesis for Pearson's chi-square goodness-of-fit is that the two categorized samples have the same distribution. For Pearson's chi-square goodness-of-fit test, we categorize the simulated data into three groups: less than zero, zero and greater than zero. The bold entries are the $p$-values smaller than $5 \%$. 
Table 10: Empirical and Simulated Autocorrelation Functions

\begin{tabular}{|c|c|c|c|c|c|c|c|c|c|c|}
\hline \multirow[b]{2}{*}{ Ticker } & \multicolumn{5}{|c|}{ Empirical ACFs } & \multicolumn{5}{|c|}{ Simulated ACFs } \\
\hline & $\rho_{1}$ & $\rho_{2}$ & $\rho_{3}$ & $\rho_{4}$ & $\rho_{5}$ & $\hat{\rho}_{1}$ & $\hat{\rho}_{2}$ & $\hat{\rho}_{3}$ & $\hat{\rho}_{4}$ & $\hat{\rho}_{5}$ \\
\hline ADS & $-0.205^{*}$ & $-0.085^{*}$ & $-0.076^{*}$ & -0.003 & $-0.029 *$ & $-0.145^{*}$ & $-0.078^{*}$ & $-0.049^{*}$ & $-0.031^{*}$ & -0.021 \\
\hline ALV & $-0.264^{*}$ & $-0.087^{*}$ & $-0.045^{*}$ & $-0.012^{*}$ & -0.006 & $-0.260 *$ & $-0.076^{*}$ & $-0.035^{*}$ & $-0.018^{*}$ & -0.010 \\
\hline BAS & $-0.229^{*}$ & $-0.122^{*}$ & $-0.038^{*}$ & -0.006 & $-0.059^{*}$ & $-0.239^{*}$ & $-0.087^{*}$ & $-0.041^{*}$ & $-0.021^{*}$ & -0.011 \\
\hline BAYN & $-0.281^{*}$ & $-0.107^{*}$ & $-0.035^{*}$ & -0.005 & $-0.022^{*}$ & $-0.255^{*}$ & $-0.081^{*}$ & $-0.037^{*}$ & $-0.019 *$ & -0.011 \\
\hline BEI & $-0.170 *$ & $-0.089^{*}$ & $-0.042^{*}$ & $-0.052^{*}$ & $-0.055^{*}$ & $-0.160 *$ & $-0.078^{*}$ & $-0.044^{*}$ & -0.027 & -0.018 \\
\hline BMW & $-0.245^{*}$ & $-0.060 *$ & $-0.038^{*}$ & $-0.067^{*}$ & $-0.013^{*}$ & $-0.220^{*}$ & $-0.087^{*}$ & $-0.042^{*}$ & $-0.022^{*}$ & -0.013 \\
\hline CBK & $-0.206^{*}$ & $-0.117^{*}$ & $-0.063^{*}$ & $-0.026^{*}$ & $-0.029 *$ & $-0.143^{*}$ & $-0.076^{*}$ & $-0.045^{*}$ & $-0.027^{*}$ & $-0.017 *$ \\
\hline DAI & $-0.274^{*}$ & $-0.089^{*}$ & $-0.031^{*}$ & $-0.022^{*}$ & $-0.023^{*}$ & $-0.248^{*}$ & $-0.086^{*}$ & $-0.041 *$ & $-0.022 *$ & -0.012 \\
\hline DB1 & $-0.225^{*}$ & $-0.123^{*}$ & $-0.057^{*}$ & -0.013 & $-0.015^{*}$ & $-0.176^{*}$ & $-0.082^{*}$ & $-0.044^{*}$ & $-0.026^{*}$ & -0.017 \\
\hline DBK & $-0.241 *$ & $-0.090 *$ & $-0.023^{*}$ & $-0.025^{*}$ & $-0.031^{*}$ & $-0.244^{*}$ & $-0.082^{*}$ & $-0.040^{*}$ & $-0.021^{*}$ & -0.011 \\
\hline DPW & $-0.226^{*}$ & $-0.126^{*}$ & $-0.035^{*}$ & -0.016 & -0.008 & $-0.182^{*}$ & $-0.085^{*}$ & $-0.043^{*}$ & -0.023 & -0.013 \\
\hline DTE & $-0.244^{*}$ & $-0.117^{*}$ & $-0.051^{*}$ & $-0.023^{*}$ & -0.013 & $-0.239^{*}$ & $-0.087^{*}$ & $-0.033^{*}$ & -0.013 & -0.006 \\
\hline EOAN & $-0.295^{*}$ & $-0.105^{*}$ & $-0.018^{*}$ & $-0.013^{*}$ & $-0.015^{*}$ & $-0.285^{*}$ & $-0.077^{*}$ & $-0.034^{*}$ & -0.017 & -0.008 \\
\hline FME & $-0.203^{*}$ & $-0.125^{*}$ & $-0.044^{*}$ & $-0.020^{*}$ & $-0.039^{*}$ & $-0.171^{*}$ & $-0.082^{*}$ & $-0.048^{*}$ & $-0.030^{*}$ & -0.017 \\
\hline FRE & $-0.137^{*}$ & $-0.138^{*}$ & $-0.069^{*}$ & $-0.032^{*}$ & $-0.032^{*}$ & $-0.107^{*}$ & $-0.083^{*}$ & $-0.052^{*}$ & $-0.033^{*}$ & -0.020 \\
\hline HEI & $-0.236^{*}$ & $-0.100 *$ & $-0.058^{*}$ & $-0.021^{*}$ & 0.006 & $-0.165^{*}$ & $-0.082^{*}$ & $-0.044^{*}$ & $-0.025^{*}$ & -0.015 \\
\hline HEN3 & $-0.209 *$ & $-0.109^{*}$ & $-0.049^{*}$ & -0.005 & $-0.026^{*}$ & $-0.173^{*}$ & $-0.082^{*}$ & $-0.046^{*}$ & $-0.027^{*}$ & -0.017 \\
\hline IFX & $-0.225 *$ & $-0.130^{*}$ & $-0.030^{*}$ & $-0.024^{*}$ & $-0.030 *$ & $-0.171^{*}$ & $-0.077^{*}$ & $-0.041^{*}$ & $-0.024^{*}$ & -0.015 \\
\hline LHA & $-0.214^{*}$ & $-0.117^{*}$ & $-0.046^{*}$ & $-0.041^{*}$ & $-0.024^{*}$ & $-0.134^{*}$ & $-0.076^{*}$ & $-0.047^{*}$ & $-0.029 *$ & -0.018 \\
\hline LIN & $-0.261^{*}$ & $-0.103^{*}$ & $-0.060 *$ & $-0.024^{*}$ & -0.004 & $-0.175^{*}$ & $-0.085^{*}$ & $-0.047^{*}$ & -0.026 & -0.015 \\
\hline MAN & $-0.289^{*}$ & $-0.057^{*}$ & $-0.053^{*}$ & $-0.015^{*}$ & $-0.038^{*}$ & $-0.281^{*}$ & $-0.074^{*}$ & $-0.035^{*}$ & -0.020 & -0.012 \\
\hline MEO & $-0.252^{*}$ & $-0.113^{*}$ & $-0.030 *$ & -0.010 & -0.015 & $-0.186^{*}$ & $-0.083^{*}$ & $-0.045^{*}$ & $-0.026^{*}$ & -0.016 \\
\hline MRK & $-0.226^{*}$ & $-0.095^{*}$ & -0.001 & $-0.059 *$ & $-0.027^{*}$ & $-0.176^{*}$ & $-0.083^{*}$ & $-0.045^{*}$ & $-0.024 *$ & -0.014 \\
\hline MUV2 & $-0.269^{*}$ & $-0.103^{*}$ & $-0.040 *$ & $-0.023^{*}$ & -0.018 & $-0.238^{*}$ & $-0.085^{*}$ & $-0.032^{*}$ & -0.013 & -0.006 \\
\hline RWE & $-0.175^{*}$ & $-0.055^{*}$ & $-0.022^{*}$ & -0.011 & $0.028^{*}$ & $-0.244^{*}$ & $-0.084^{*}$ & $-0.040^{*}$ & $-0.021^{*}$ & -0.011 \\
\hline SAP & $-0.274^{*}$ & $-0.116^{*}$ & $-0.028^{*}$ & $-0.011^{*}$ & $-0.033^{*}$ & $-0.271^{*}$ & $-0.084^{*}$ & $-0.037^{*}$ & $-0.019 *$ & -0.010 \\
\hline SDF & $-0.233^{*}$ & $-0.106^{*}$ & $-0.053^{*}$ & $-0.022^{*}$ & $-0.026^{*}$ & $-0.168^{*}$ & $-0.086^{*}$ & $-0.049^{*}$ & $-0.029 *$ & -0.017 \\
\hline SIE & $-0.286^{*}$ & $-0.088^{*}$ & $-0.032^{*}$ & $-0.047^{*}$ & $0.010^{*}$ & $-0.282^{*}$ & $-0.077^{*}$ & $-0.032^{*}$ & $-0.016^{*}$ & -0.009 \\
\hline TKA & $-0.255^{*}$ & $-0.120 *$ & $-0.015^{*}$ & $-0.045^{*}$ & -0.004 & $-0.186^{*}$ & $-0.087^{*}$ & $-0.044^{*}$ & $-0.025^{*}$ & -0.015 \\
\hline VOW3 & $-0.202^{*}$ & $-0.103^{*}$ & $-0.042^{*}$ & $-0.021 *$ & $-0.028^{*}$ & $-0.140 *$ & $-0.076^{*}$ & $-0.045^{*}$ & $-0.027^{*}$ & -0.017 \\
\hline Mean & -0.235 & -0.103 & -0.041 & -0.024 & -0.021 & -0.202 & -0.082 & -0.042 & -0.023 & -0.014 \\
\hline Median & -0.234 & -0.105 & -0.041 & -0.021 & -0.024 & -0.184 & -0.082 & -0.043 & -0.024 & -0.014 \\
\hline Min & -0.295 & -0.138 & -0.076 & -0.067 & -0.059 & -0.285 & -0.087 & -0.052 & -0.033 & -0.021 \\
\hline Max & -0.137 & -0.055 & -0.001 & -0.003 & 0.028 & -0.107 & -0.074 & -0.032 & -0.013 & -0.006 \\
\hline
\end{tabular}

The table presents the empirical and simulated ACFs from 1 to 5 lags $\left(\rho_{1} \ldots \rho_{5}\right)$ for 30 stocks in the third week of May 2011. A given simulated ACF is the average of the ACFs for 5000 paths of simulations. * denote the autocorrelation coefficients that are significant at the $5 \%$. 
Table 11: Out-of-sample MSE for Various Horizons

\begin{tabular}{|c|c|c|c|c|c|c|c|}
\hline \multirow{2}{*}{ Tickers } & \multicolumn{7}{|c|}{ Interval (ticks) } \\
\hline & 5 & 10 & 30 & 50 & 100 & 150 & 200 \\
\hline ADS & 0.30 & 0.73 & 1.72 & 2.26 & 3.55 & 5.74 & 8.94 \\
\hline ALV & 0.16 & 0.43 & 2.20 & 3.49 & 5.06 & 7.50 & 11.33 \\
\hline BAS & 0.14 & 0.38 & 2.03 & 3.66 & 5.50 & 8.01 & 11.84 \\
\hline BAYN & 0.14 & 0.35 & 1.41 & 1.86 & 2.72 & 4.15 & 6.07 \\
\hline BEI & 0.45 & 1.13 & 1.96 & 2.69 & 4.65 & 8.54 & 16.38 \\
\hline BMW & 0.20 & 0.52 & 2.68 & 3.58 & 5.28 & 7.74 & 11.48 \\
\hline CBK & 0.12 & 0.32 & 1.62 & 3.61 & 5.66 & 8.87 & 14.91 \\
\hline DAI & 0.11 & 0.28 & 1.42 & 2.50 & 3.75 & 5.59 & 8.63 \\
\hline DB1 & 0.24 & 0.62 & 2.38 & 3.14 & 4.77 & 7.22 & 11.13 \\
\hline DBK & 0.08 & 0.20 & 1.00 & 2.01 & 3.04 & 4.50 & 6.82 \\
\hline DPW & 0.44 & 1.16 & 2.97 & 4.02 & 5.89 & 9.08 & 13.28 \\
\hline DTE & 0.24 & 0.64 & 2.25 & 3.09 & 4.70 & 7.24 & 11.98 \\
\hline EOAN & 0.15 & 0.40 & 1.87 & 2.49 & 3.67 & 5.44 & 8.26 \\
\hline FME & 0.38 & 1.00 & 2.64 & 3.65 & 6.16 & 10.48 & 21.06 \\
\hline FRE & 0.33 & 0.83 & 2.19 & 2.96 & 5.10 & 10.37 & 22.62 \\
\hline HEI & 0.32 & 0.85 & 3.42 & 4.68 & 7.48 & 12.39 & 22.46 \\
\hline HEN3 & 0.28 & 0.69 & 1.77 & 2.45 & 3.98 & 6.90 & 12.99 \\
\hline IFX & 0.16 & 0.39 & 1.66 & 2.20 & 3.37 & 5.46 & 9.56 \\
\hline LHA & 0.35 & 0.87 & 2.15 & 2.98 & 4.56 & 6.43 & 10.36 \\
\hline LIN & 0.49 & 1.20 & 2.01 & 2.69 & 4.06 & 5.92 & 9.07 \\
\hline MAN & 0.24 & 0.64 & 2.28 & 3.03 & 4.80 & 7.41 & 13.16 \\
\hline MEO & 0.35 & 0.90 & 2.34 & 3.22 & 4.93 & 8.40 & 14.27 \\
\hline MRK & 0.37 & 0.92 & 2.37 & 3.02 & 4.62 & 7.39 & 11.78 \\
\hline MUV2 & 0.49 & 1.29 & 2.66 & 3.55 & 5.34 & 7.77 & 11.17 \\
\hline RWE & 0.15 & 0.37 & 1.36 & 1.92 & 2.69 & 4.13 & 6.23 \\
\hline SAP & 0.12 & 0.33 & 1.65 & 2.28 & 3.37 & 4.99 & 7.53 \\
\hline $\mathrm{SDF}$ & 0.21 & 0.49 & 1.31 & 1.63 & 2.56 & 4.10 & 6.71 \\
\hline SIE & 0.08 & 0.19 & 0.94 & 1.84 & 2.73 & 4.12 & 6.26 \\
\hline TKA & 0.19 & 0.50 & 2.33 & 3.15 & 4.81 & 7.22 & 11.32 \\
\hline VOW3 & 0.29 & 0.72 & 2.04 & 2.69 & 4.17 & 6.53 & 9.84 \\
\hline Mean & 0.25 & 0.64 & 2.02 & 2.88 & 4.43 & 6.99 & 11.58 \\
\hline Median & 0.24 & 0.63 & 2.03 & 2.97 & 4.64 & 7.22 & 11.25 \\
\hline Min & 0.08 & 0.19 & 0.94 & 1.63 & 2.56 & 4.10 & 6.07 \\
\hline $\operatorname{Max}$ & 0.49 & 1.29 & 3.42 & 4.68 & 7.48 & 12.39 & 22.62 \\
\hline
\end{tabular}

The table presents the Out-of-sample mean squared error (MSE) expressed in basis points for 30 stocks in DAX30 for May 2011. Note that the mean of the EWS changes is around zero and the standard deviation is around one. The average real time for 1 tick is around 7 seconds. 


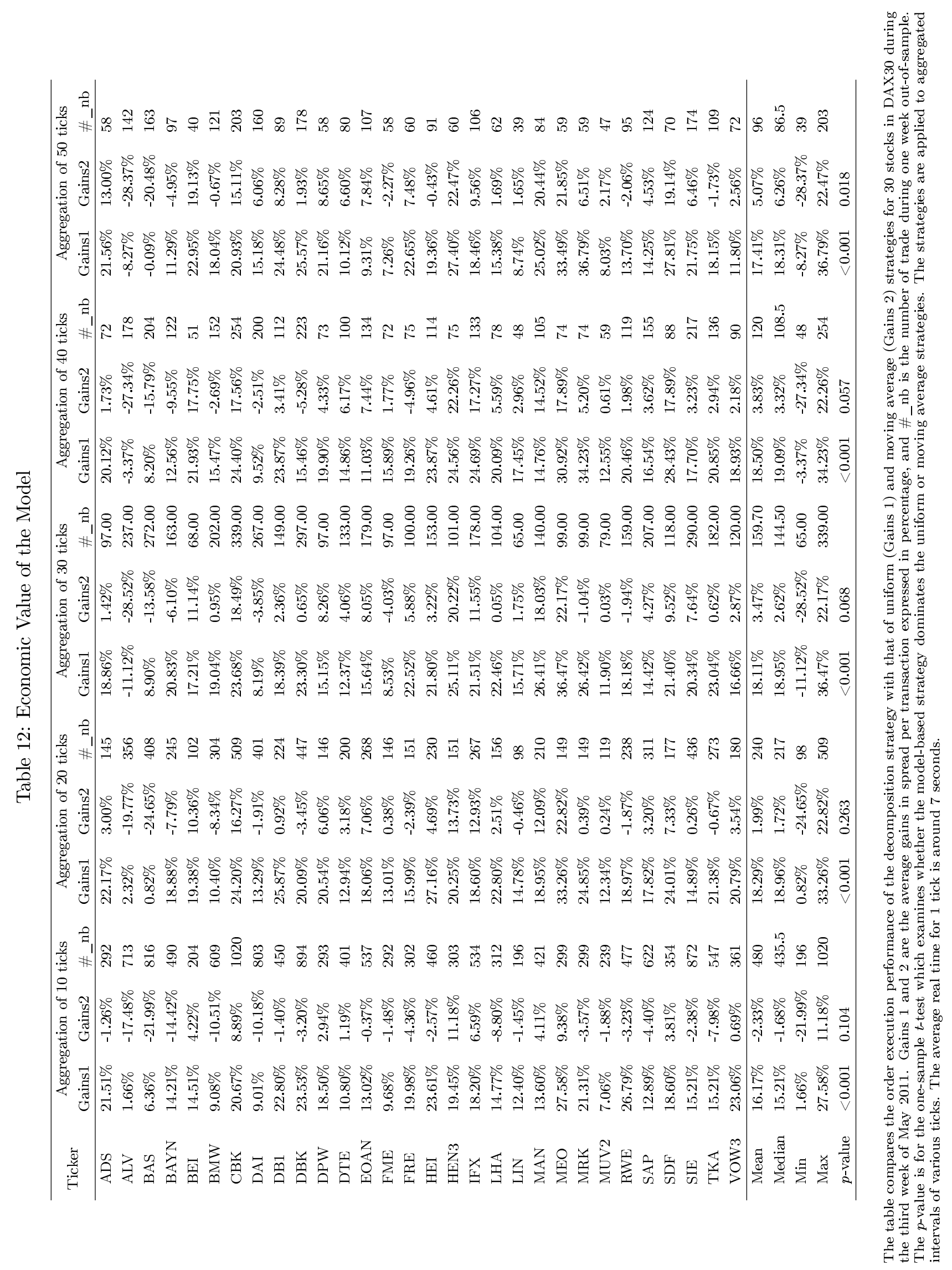


Table 13: Empirical and Simulated Time-Based Resilience

\begin{tabular}{|c|c|c|c|c|c|c|c|c|c|c|}
\hline \multirow[t]{2}{*}{ Ticker } & \multicolumn{2}{|c|}{ 1-BPS Shock } & \multicolumn{2}{|c|}{ 2-BPS Shock } & \multicolumn{2}{|c|}{ 3-BPS Shock } & \multicolumn{2}{|c|}{ 4-BPS Shock } & \multicolumn{2}{|c|}{ 5-BPS Shock } \\
\hline & Empir. & Sim. & Empir. & Sim. & Empir. & Sim. & Empir. & Sim. & Empir. & Sim. \\
\hline ADS & 11 & 14 & 17 & 22 & 23 & 31 & 27 & 39 & 32 & 46 \\
\hline ALV & 10 & 19 & 19 & 31 & 37 & 42 & 20 & 52 & 23 & 61 \\
\hline BAS & 10 & 9 & 20 & 16 & 35 & 23 & 39 & 30 & 52 & 36 \\
\hline BAYN & 18 & 10 & 29 & 19 & 45 & 26 & 55 & 35 & 71 & 43 \\
\hline BEI & 8 & 10 & 12 & 17 & 17 & 23 & 21 & 29 & 25 & 35 \\
\hline BMW & 10 & 11 & 20 & 19 & 28 & 27 & 36 & 34 & 44 & 41 \\
\hline CBK & 11 & 12 & 16 & 20 & 21 & 27 & 27 & 33 & 33 & 39 \\
\hline DAI & 10 & 10 & 18 & 17 & 25 & 24 & 35 & 31 & 42 & 37 \\
\hline DB1 & 12 & 17 & 19 & 26 & 27 & 36 & 34 & 45 & 34 & 52 \\
\hline DBK & 14 & 10 & 29 & 18 & 42 & 25 & 43 & 32 & 44 & 39 \\
\hline DPW & 9 & 11 & 14 & 18 & 19 & 25 & 28 & 31 & 30 & 38 \\
\hline DTE & 9 & 11 & 15 & 20 & 18 & 27 & 23 & 35 & 30 & 42 \\
\hline EOAN & 8 & 9 & 14 & 16 & 20 & 23 & 22 & 30 & 32 & 37 \\
\hline FME & 10 & 17 & 16 & 27 & 22 & 37 & 27 & 43 & 32 & 51 \\
\hline FRE & 12 & 16 & 15 & 24 & 19 & 33 & 23 & 40 & 30 & 47 \\
\hline HEI & 10 & 19 & 17 & 30 & 26 & 40 & 34 & 48 & 39 & 56 \\
\hline HEN3 & 13 & 16 & 20 & 25 & 28 & 35 & 35 & 44 & 39 & 51 \\
\hline IFX & 9 & 12 & 15 & 19 & 21 & 26 & 25 & 33 & 29 & 40 \\
\hline LHA & 10 & 11 & 15 & 17 & 20 & 23 & 25 & 30 & 35 & 35 \\
\hline LIN & 7 & 8 & 10 & 13 & 12 & 18 & 14 & 23 & 18 & 28 \\
\hline MAN & 21 & 23 & 33 & 36 & 47 & 48 & 61 & 58 & 69 & 66 \\
\hline MEO & 14 & 13 & 21 & 21 & 26 & 29 & 31 & 37 & 27 & 44 \\
\hline MRK & 14 & 14 & 23 & 22 & 32 & 30 & 43 & 37 & 53 & 44 \\
\hline MUV2 & 5 & 8 & 8 & 13 & 16 & 18 & 28 & 23 & 28 & 28 \\
\hline RWE & 14 & 16 & 25 & 26 & 36 & 35 & 42 & 44 & 49 & 53 \\
\hline SAP & 20 & 10 & 34 & 19 & 53 & 27 & 87 & 35 & 121 & 43 \\
\hline SDF & 10 & 13 & 15 & 21 & 20 & 28 & 26 & 36 & 29 & 43 \\
\hline SIE & 20 & 10 & 28 & 18 & 39 & 26 & 56 & 34 & 57 & 41 \\
\hline TKA & 13 & 13 & 21 & 20 & 32 & 28 & 40 & 34 & 44 & 41 \\
\hline VOW3 & 10 & 8 & 14 & 13 & 18 & 17 & 23 & 22 & 28 & 26 \\
\hline Mean & 11.7 & 12.6 & 19.0 & 20.8 & 27.5 & 28.6 & 34.4 & 35.9 & 40.7 & 42.8 \\
\hline Median & 10 & 12 & 17 & 19 & 25 & 27 & 30 & 35 & 34 & 42 \\
\hline Min & 5 & 8 & 8 & 13 & 12 & 17 & 14 & 22 & 18 & 26 \\
\hline Max & 21 & 23 & 34 & 36 & 53 & 48 & 87 & 58 & 121 & 66 \\
\hline
\end{tabular}

The table presents the empirical and simulated time-based resilience, measured in ticks, of the higher-level of the LOB for the 30 stocks in DAX during May 2011. The empirical time-based resilience is measured by the number of ticks with which liquidity reverts to its reference state from a given liquidity shock. The simulated time-based resilience is the average of reversion time for all simulated paths. The average real time for 1 tick is around 7 seconds. 
Table 14: Empirical and Simulated Probability-Based Resilience

\begin{tabular}{|c|c|c|c|c|c|c|c|c|c|c|c|c|}
\hline \multirow[b]{3}{*}{ Ticker } & \multicolumn{6}{|c|}{ 1-BPS Shock } & \multicolumn{6}{|c|}{ 3-BPS Shock } \\
\hline & \multicolumn{2}{|c|}{5 tick } & \multicolumn{2}{|c|}{10 tick } & \multicolumn{2}{|c|}{30 ticks } & \multicolumn{2}{|c|}{5 tick } & \multicolumn{2}{|c|}{10 ticks } & \multicolumn{2}{|c|}{30 ticks } \\
\hline & Empir. & Sim. & Empir. & Sim. & Empir. & Sim. & Empir. & Sim. & Empir. & Sim. & Empir. & Sim. \\
\hline ADS & $50.57 \%$ & $46.38 \%$ & $69.38 \%$ & $56.50 \%$ & $92.03 \%$ & $71.92 \%$ & $20.71 \%$ & $8.24 \%$ & $39.69 \%$ & $21.68 \%$ & $74.96 \%$ & $51.70 \%$ \\
\hline ALV & $37.36 \%$ & $36.54 \%$ & $49.72 \%$ & $44.94 \%$ & $63.27 \%$ & $56.80 \%$ & $1.84 \%$ & $4.44 \%$ & $3.76 \%$ & $11.92 \%$ & $9.29 \%$ & $32.92 \%$ \\
\hline BAS & $52.58 \%$ & $54.44 \%$ & $71.82 \%$ & $67.44 \%$ & $93.14 \%$ & $85.86 \%$ & $17.54 \%$ & $11.28 \%$ & $32.08 \%$ & $29.80 \%$ & $67.24 \%$ & $69.46 \%$ \\
\hline BAYN & $37.81 \%$ & $50.36 \%$ & $54.94 \%$ & $62.78 \%$ & $82.55 \%$ & $80.66 \%$ & $10.40 \%$ & $7.52 \%$ & $21.62 \%$ & $22.96 \%$ & $51.33 \%$ & $60.04 \%$ \\
\hline BEI & $57.00 \%$ & $52.68 \%$ & $75.44 \%$ & $62.94 \%$ & $95.94 \%$ & $78.56 \%$ & $26.56 \%$ & $15.22 \%$ & $48.76 \%$ & $32.82 \%$ & $84.57 \%$ & $63.50 \%$ \\
\hline BMW & $54.48 \%$ & $52.02 \%$ & $72.82 \%$ & $61.96 \%$ & $93.35 \%$ & $78.38 \%$ & $16.97 \%$ & $11.06 \%$ & $33.97 \%$ & $26.64 \%$ & $71.33 \%$ & $59.54 \%$ \\
\hline CBK & $51.34 \%$ & $49.64 \%$ & $69.74 \%$ & $57.46 \%$ & $91.56 \%$ & $69.38 \%$ & $21.46 \%$ & $15.68 \%$ & $40.70 \%$ & $30.68 \%$ & $78.88 \%$ & $54.36 \%$ \\
\hline DAI & $54.80 \%$ & $52.60 \%$ & $73.62 \%$ & $64.52 \%$ & $93.50 \%$ & $80.68 \%$ & $17.85 \%$ & $11.32 \%$ & $33.42 \%$ & $29.10 \%$ & $71.55 \%$ & $63.68 \%$ \\
\hline DB1 & $50.20 \%$ & $42.08 \%$ & $69.56 \%$ & $52.56 \%$ & $92.11 \%$ & $66.70 \%$ & $20.43 \%$ & $7.06 \%$ & $37.34 \%$ & $17.38 \%$ & $73.54 \%$ & $43.78 \%$ \\
\hline DBK & $48.05 \%$ & $51.66 \%$ & $66.06 \%$ & $62.94 \%$ & $88.60 \%$ & $79.40 \%$ & $12.95 \%$ & $11.56 \%$ & $25.41 \%$ & $28.02 \%$ & $58.21 \%$ & $61.98 \%$ \\
\hline DPW & $52.21 \%$ & $51.78 \%$ & $73.02 \%$ & $62.06 \%$ & $94.79 \%$ & $77.16 \%$ & $25.88 \%$ & $15.18 \%$ & $45.94 \%$ & $31.14 \%$ & $84.47 \%$ & $61.48 \%$ \\
\hline DTE & $51.28 \%$ & $50.72 \%$ & $72.29 \%$ & $62.36 \%$ & $95.50 \%$ & $80.14 \%$ & $26.13 \%$ & $9.52 \%$ & $48.65 \%$ & $24.82 \%$ & $82.66 \%$ & $60.22 \%$ \\
\hline EOAN & $61.52 \%$ & $53.30 \%$ & $79.14 \%$ & $67.04 \%$ & $95.49 \%$ & $86.34 \%$ & $19.40 \%$ & $9.12 \%$ & $39.02 \%$ & $25.62 \%$ & $79.60 \%$ & $69.00 \%$ \\
\hline FME & $54.87 \%$ & $41.94 \%$ & $72.51 \%$ & $47.52 \%$ & $93.22 \%$ & $54.92 \%$ & $20.02 \%$ & $7.96 \%$ & $39.15 \%$ & $18.68 \%$ & $76.25 \%$ & $36.58 \%$ \\
\hline FRE & $45.81 \%$ & $45.44 \%$ & $64.93 \%$ & $52.50 \%$ & $91.27 \%$ & $64.38 \%$ & $21.33 \%$ & $12.08 \%$ & $41.24 \%$ & $24.34 \%$ & $82.07 \%$ & $47.40 \%$ \\
\hline HEI & $52.14 \%$ & $39.06 \%$ & $70.83 \%$ & $47.54 \%$ & $93.21 \%$ & $59.10 \%$ & $20.72 \%$ & $6.66 \%$ & $38.26 \%$ & $15.80 \%$ & $74.79 \%$ & $37.06 \%$ \\
\hline HEN3 & $48.35 \%$ & $43.50 \%$ & $65.07 \%$ & $54.24 \%$ & $89.08 \%$ & $66.62 \%$ & $15.46 \%$ & $7.92 \%$ & $31.47 \%$ & $18.42 \%$ & $68.60 \%$ & $46.16 \%$ \\
\hline IFX & $55.85 \%$ & $51.68 \%$ & $74.51 \%$ & $60.56 \%$ & $95.08 \%$ & $75.40 \%$ & $21.37 \%$ & $14.60 \%$ & $41.32 \%$ & $30.56 \%$ & $80.20 \%$ & $59.20 \%$ \\
\hline LHA & $51.89 \%$ & $52.54 \%$ & $70.96 \%$ & $61.58 \%$ & $93.48 \%$ & $74.28 \%$ & $22.03 \%$ & $18.70 \%$ & $41.15 \%$ & $35.60 \%$ & $80.04 \%$ & $61.64 \%$ \\
\hline LIN & $61.87 \%$ & $60.70 \%$ & $81.48 \%$ & $69.44 \%$ & $97.41 \%$ & $85.24 \%$ & $36.63 \%$ & $23.22 \%$ & $60.86 \%$ & $44.26 \%$ & $92.95 \%$ & $74.52 \%$ \\
\hline MAN & $37.50 \%$ & $31.22 \%$ & $53.64 \%$ & $42.02 \%$ & $79.56 \%$ & $53.38 \%$ & $11.36 \%$ & $3.60 \%$ & $23.22 \%$ & $8.72 \%$ & $52.93 \%$ & $27.04 \%$ \\
\hline MEO & $44.17 \%$ & $48.88 \%$ & $62.07 \%$ & $58.02 \%$ & $87.33 \%$ & $72.74 \%$ & $17.18 \%$ & $10.58 \%$ & $34.02 \%$ & $24.86 \%$ & $70.88 \%$ & $54.28 \%$ \\
\hline MRK & $47.39 \%$ & $46.82 \%$ & $63.56 \%$ & $54.06 \%$ & $87.31 \%$ & $65.82 \%$ & $15.90 \%$ & $12.88 \%$ & $32.70 \%$ & $25.54 \%$ & $67.99 \%$ & $50.20 \%$ \\
\hline MUV2 & $71.87 \%$ & $59.94 \%$ & $89.46 \%$ & $70.36 \%$ & $98.88 \%$ & $86.54 \%$ & $34.58 \%$ & $18.64 \%$ & $54.91 \%$ & $40.18 \%$ & $84.58 \%$ & $75.62 \%$ \\
\hline RWE & $44.82 \%$ & $46.10 \%$ & $62.66 \%$ & $54.40 \%$ & $87.73 \%$ & $68.48 \%$ & $11.86 \%$ & $11.12 \%$ & $23.51 \%$ & $19.78 \%$ & $54.75 \%$ & $45.60 \%$ \\
\hline SAP & $36.36 \%$ & $48.26 \%$ & $53.67 \%$ & $60.48 \%$ & $80.75 \%$ & $79.24 \%$ & $9.25 \%$ & $7.02 \%$ & $18.97 \%$ & $21.58 \%$ & $47.93 \%$ & $57.76 \%$ \\
\hline SDF & $50.63 \%$ & $49.80 \%$ & $69.14 \%$ & $58.90 \%$ & $92.88 \%$ & $72.96 \%$ & $20.19 \%$ & $12.26 \%$ & $39.29 \%$ & $26.42 \%$ & $78.43 \%$ & $55.64 \%$ \\
\hline SIE & $30.69 \%$ & $50.38 \%$ & $47.65 \%$ & $63.24 \%$ & $79.86 \%$ & $81.88 \%$ & $11.71 \%$ & $9.02 \%$ & $24.01 \%$ & $24.90 \%$ & $55.82 \%$ & $61.90 \%$ \\
\hline TKA & $48.79 \%$ & $50.02 \%$ & $67.11 \%$ & $59.60 \%$ & $90.15 \%$ & $73.34 \%$ & $16.52 \%$ & $13.60 \%$ & $33.54 \%$ & $29.78 \%$ & $68.72 \%$ & $57.06 \%$ \\
\hline VOW3 & $51.65 \%$ & $59.02 \%$ & $70.87 \%$ & $71.08 \%$ & $93.32 \%$ & $86.62 \%$ & $23.51 \%$ & $25.54 \%$ & $44.13 \%$ & $45.74 \%$ & $82.61 \%$ & $77.08 \%$ \\
\hline Mean & $49.79 \%$ & $48.98 \%$ & $67.92 \%$ & $59.03 \%$ & $90.08 \%$ & $73.76 \%$ & $18.92 \%$ & $11.75 \%$ & $35.74 \%$ & $26.26 \%$ & $70.24 \%$ & $55.88 \%$ \\
\hline Median & $50.96 \%$ & $50.19 \%$ & $69.65 \%$ & $60.52 \%$ & $92.50 \%$ & $74.84 \%$ & $19.71 \%$ & $11.20 \%$ & $37.80 \%$ & $25.58 \%$ & $74.17 \%$ & $58.48 \%$ \\
\hline Min & $30.69 \%$ & $31.22 \%$ & $47.65 \%$ & $42.02 \%$ & $63.27 \%$ & $53.38 \%$ & $1.84 \%$ & $3.60 \%$ & $3.76 \%$ & $8.72 \%$ & $9.29 \%$ & $27.04 \%$ \\
\hline $\operatorname{Max}$ & $71.87 \%$ & $60.70 \%$ & $89.46 \%$ & $71.08 \%$ & $98.88 \%$ & $86.62 \%$ & $36.63 \%$ & $25.54 \%$ & $60.86 \%$ & $45.74 \%$ & $92.95 \%$ & $77.08 \%$ \\
\hline
\end{tabular}

The table presents the empirical and probability-based resilience of the higher-level of the LOB for the 30 stocks in DAX during May 2011. We first classify liquidity shocks according to their magnitude and calculate reversion time for all shocks with the same magnitude. Therefore, for a given magnitude of liquidity shock, we have a sample of reversion times. The empirical probability-based resilience is the value of the empirical cumulative distribution function evaluated at a given number of ticks. The simulated probability-based resilience is the number of paths where the spread reverts to its initial state for a given tick. 


\title{
Online Appendix for
}

\section{"The Dynamics of Ex-ante Weighted Spread: An Empirical Analysis"}

\author{
Georges Dionne Xiaozhou Zhou
}

\begin{abstract}
This appendix contains three sections. Section I provides further details on the reconstruction of the LOB. Section II shows the estimation results for the trade duration factor, and Section III presents the estimation results for the quote duration factor. Also, this appendix shows the intraday seasonality of trade and quote durations for all stocks in DAX30 for May 2011.
\end{abstract}




\section{Reconstruction of the LOB}

The reconstruction of the LOB is predominantly based on two main types of data streams: delta and snapshot. The delta tracks all the possible updates in the LOB such as entry, revision, cancellation and expiration, whereas the snapshot gives an overview of the state of the LOB and is sent after a constant time interval for a given stock. Xetra original data with delta and snapshot messages are first processed using the XetraParser algorithm, developed by Bilodeau (2013) in order to make Deutsche Börse Xetra raw data usable for academic and professional analysts. XetraParser reconstructs the real-time order book sequence including all the information for both auctions and continuous trading by implementing the Xetra trading protocol and Enhanced Broadcast. We then put the raw LOB information in order and in a readable format for each update time. We also retrieve useful and accurate information about the state of the LOB and the precise timestamp for order modifications and transactions during continuous trading.

\section{Trade Duration Factor}

It is well known that high-frequency duration data is characterized by seasonality. Before modeling the trade durations, we remove seasonality under a multiplicative form proposed by Anatolyev and Shakin $(2007)^{1}$ and apply the model to the deseasonalized data. Figure A.1 illustrates the evolution of the seasonality factors for trade durations.

\section{[Insert Figure A.1 here]}

Table A.2 presents the estimated results of the lagged dependent variables for all the stocks in the sample. The ACD-related models are based on the ARMA structure, meaning that the determinant parts of the dynamics of durations are specified by the lagged duration and lagged error term. As we expected, the trade durations of all stocks are highly autocorrelated. Taking the models' efficiency and parsimony into consideration, the number of lagged trade durations varies from one to three. The sum of the coefficients of lagged trade durations for all stocks is around 0.9 , which confirms a very high degree of persistence in autocorrelation. Regarding the error terms for all stocks, the means of Gamma1 and Gamma2 are 0.53 and 1.15, respectively,

\footnotetext{
${ }^{1}$ We obtain the deseasonalized durations by dividing the raw trade durations by expected trade durations, which are the interpolation value from the thirty-minute average trade durations.
} 
indicating that the distribution of the random part is abrupt and near zero; that is, expected trade duration shocks are very small.

[Insert Table A.2 here]

Considering the market-related variables mentioned above, we put four such variables in the trade duration equations: DeltaSpread, AveSpread, SquareRoot (vol), and Abs(sign.vol). Consistent with Engle and Lunde (2003), we have a positive and significant coefficient for DeltaSpread. Intuitively, when the stocks become less liquid, traders will slow their trading intensities, and trading durations will become longer. Contrary to Engle and Lunde (2003), we find a positive sign for AveSpread coefficient for most stocks. That is, the higher the AveSpread, the longer the trade durations. The difference between DeltaSpread and AveSpread is that the former captures the short-run spread change and the latter relates to the long-term spread change. This result reveals that trade intensity is sensitive for both short-run and long-run spread change. When the stocks become less liquid, traders slow down their activities and then wait until liquidity increases.

Another important market-related variable is volume-related. For all stocks, the coefficients of SquareRoot $(\mathrm{vol})$ are all negative and significant at the $1 \%$ level. This means that large trades will generate higher trading intensity and shorter trade durations. According to Easley and O'Hara (1987), large trades are likely to be related to information trade because informed traders try to exploit their information advantage by increasing both the trading intensity and trading quantity. Another volume-related variable is Abs(sign.vol) which measures the volume imbalance (in shares) of the last ten trades (a higher value means a higher level of imbalance and a zero value means a completely balanced trading activity). Most stocks have small positive coefficients at the $1 \%$ level. The measure of imbalance also relates to information trading. Our results suggest that when the trades become imbalanced, the trade intensity will decrease. Presumably, when the non-informed traders observe an imbalanced trading history, they will slow down their trading activity to protect themselves.

\section{Quote Duration Factor}

As for trade durations, quote durations also feature diurnal seasonality. Figure A.2 presents the evolution of the seasonality factors for quote durations. Table A.4 reports the estimated results of lagged dependent variables for all stocks. Similar to trade durations, quote durations are also 
highly persistent. The number of lagged values varies from two to six. Comparing the Log-ACD model with the traditional ARMA structure, the sum of the coefficient of the "AR" part in the quote duration equation is around 0.6. The relatively small coefficients can be explained by the fact that the start point of our quote duration is the trade timestamp. When there is more than one update between two trades, only the first one is used to calculate the quote duration. This sampling "deletes" some autocorrelation in quote durations. Regarding the coefficients of the error term, the estimated coefficients vary from 0.007 to 0.144 , confirming the sampling effect in autocorrelation.

[Insert Figure A.2 here]

[Insert Table A.4 here]

The censored effect is captured by the product of the censored dummy variable and the lagged error term. The censored effect is positive and significant at the $1 \%$ level for 27 stocks. The implication is that if the last quote duration is censored, the next quote duration is likely to be longer and censored again. Trade duration-related variables consist of the current error term, lagged error term and lagged expected trade duration. The estimated results show that the current error term and expected trade duration have a positive and significant effect on the quote durations. This suggests that when the (current and lagged) trade duration innovations and expected trade durations are high, the quote duration will lengthen. Intuitively, as quote activity adjusts to trade activity, trade duration and quote duration are correlated positively. AveSpread has a negative impact on the quote durations, which is the opposite of the trade duration. The coefficients are negative and significant at the $1 \%$ level for all stocks. Spread variables are very important in explaining the trade activity and quote activity. This suggests that when the average spread is large, market order traders and LOB traders react differently. Market order traders slow down their trading speed when observing an increasing spread, whereas LOB traders speed up to update their price or quantity of limit orders. For an intraday LOB trader, a major concern is adverse selection. By attentively monitoring the change in spread, LOB traders attempt to avoid this risk by updating their quote rapidly. For 22 stocks over 30, DeltaSpread has a positive impact on the quote duration, suggesting that a short-term increase in spread will reduce the quoting intensity.

The effects of the volume-related variables, SquareRoot (vol) and Abs(sign.vol), on quote duration are both negative. More specifically, large trades predict shorter quote durations. As 
mentioned above, large trades relate to informed trades. Informed traders exploit short-lived information by increasing trade intensity and trade quantity. When trades become more intensive, so do quote revisions. As a result, the quote durations shorten. The negative effect of $A b s($ sign.vol) on quote duration suggest that $A b s($ sign.vol) is more likely to be a proxy for liquidity shock than information asymmetry. When there is a need for liquidity, liquidity providers compete to provide liquidity and reduce the quote durations.

The BackQuote duration has a positive and significant impact on the following quote duration. A longer BackQuote duration can be either a low trade intensity or a high quote intensity. That is, a long trade duration or a short quote duration. Moreover, a long trade duration implies a long quote duration. The estimated results suggest that the long trade duration effect dominates the short quote duration effect. Concerning the effect of the $E W S$, it is negative and significant at the $1 \%$ level for 22 stocks. 8 stocks have a positive significant sign. Theoretically, there are arguments to support either sign. A high EWS reveals a more risky situation, especially a high adverse selection risk. LOB traders speed up their quote revisions to reduce this kind of risk. This is more pronounced for less liquid stocks. In contrast, the increase in the EWS will slow down the trade activity and then the quote activity. 


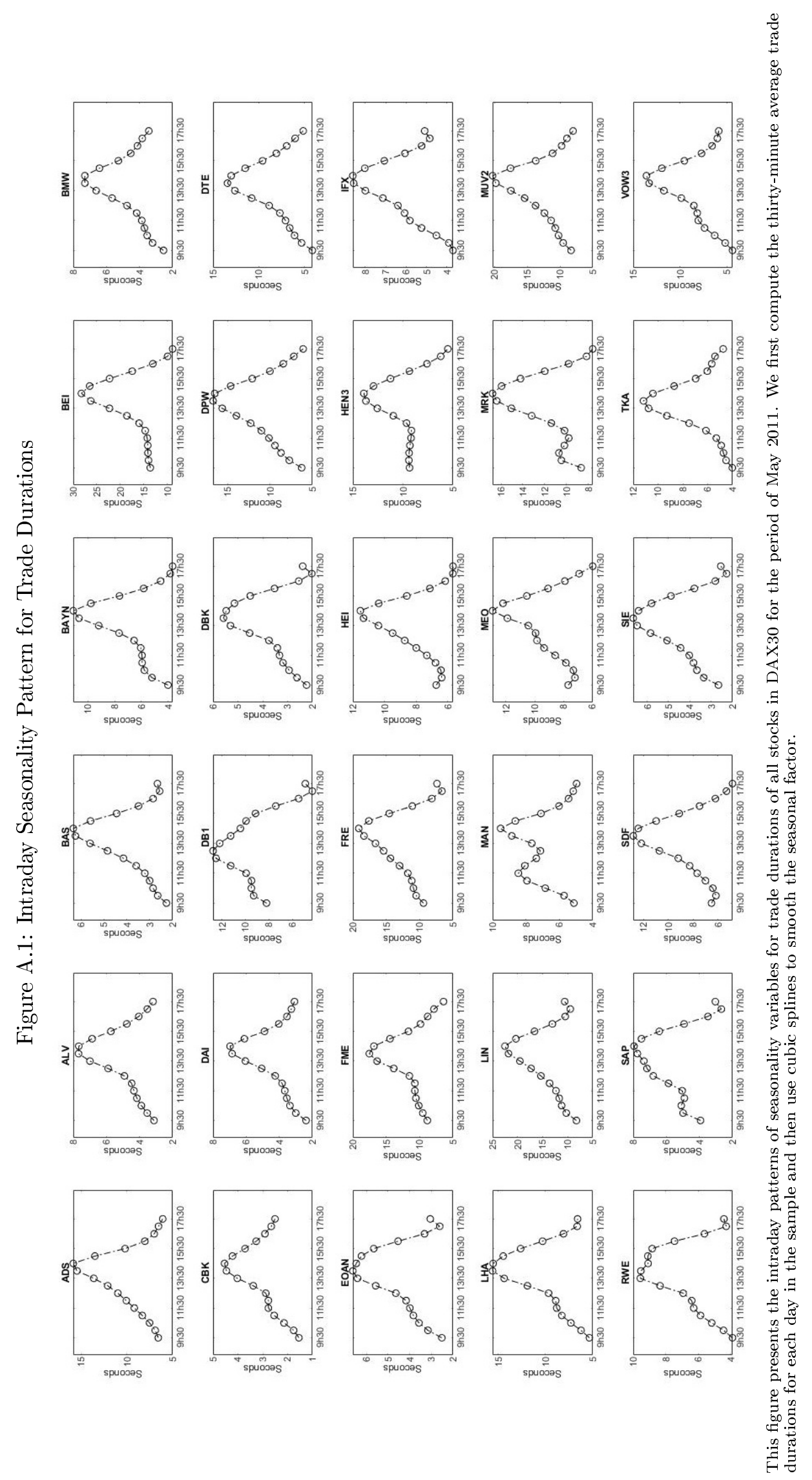




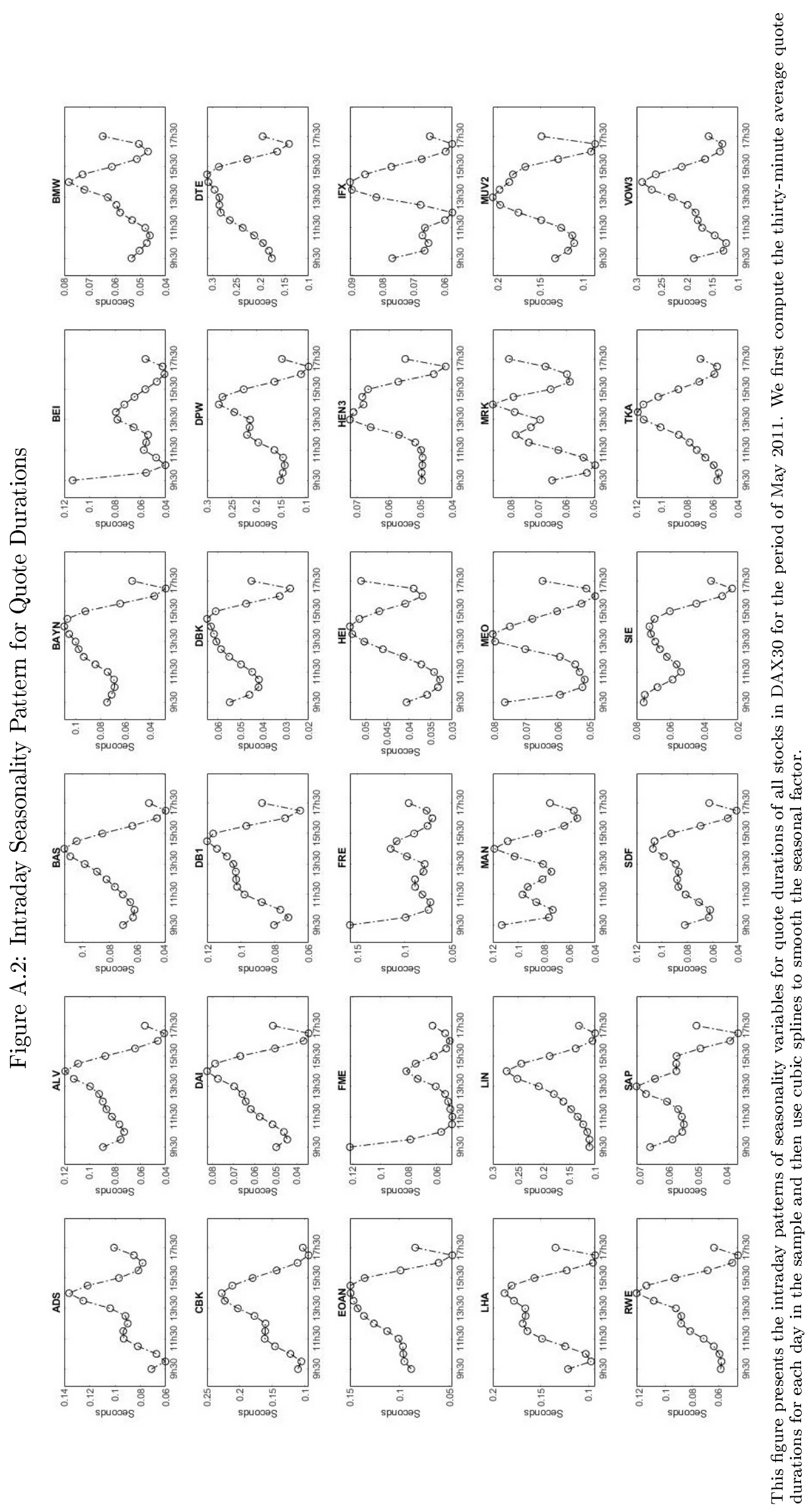




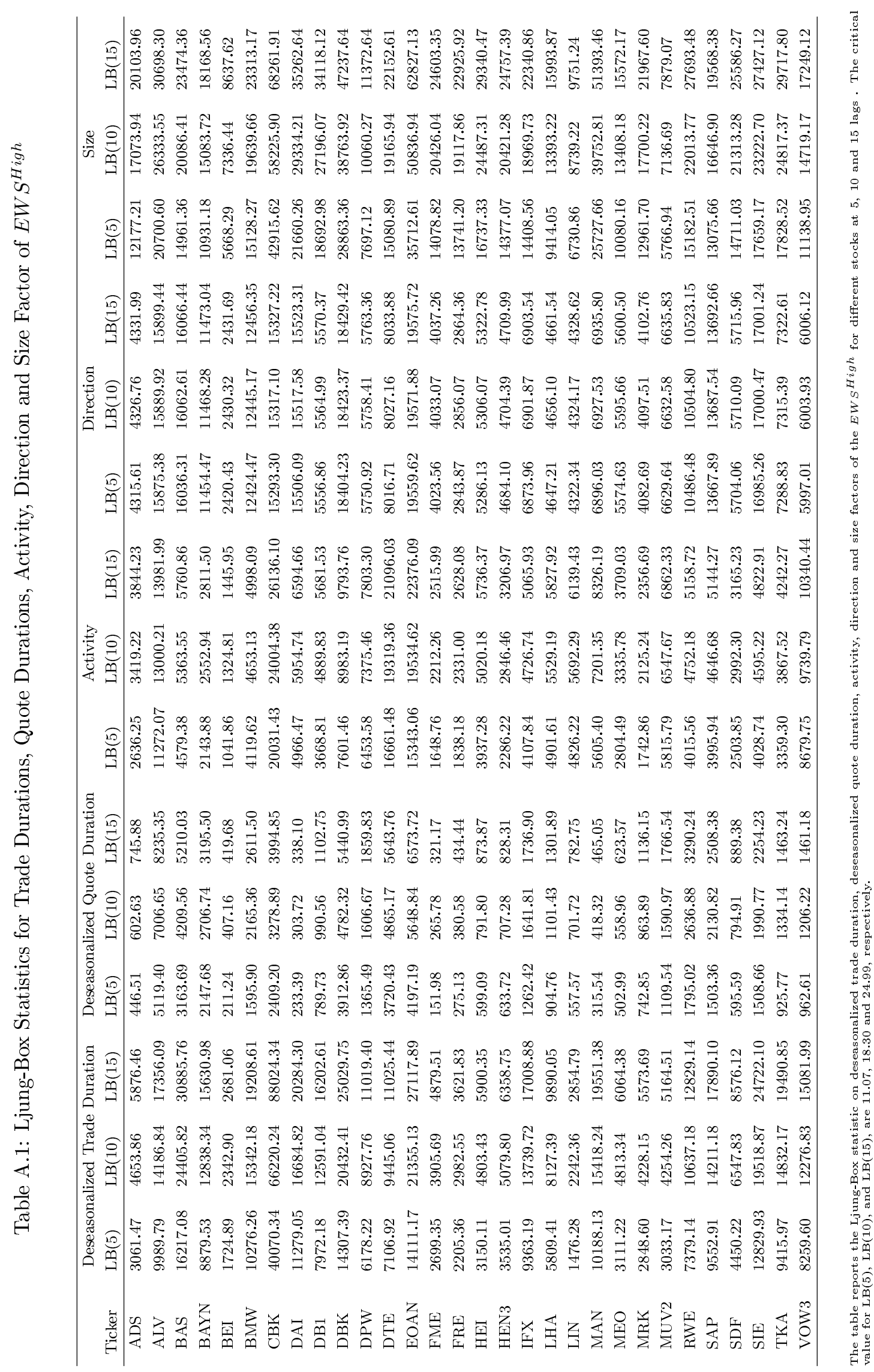




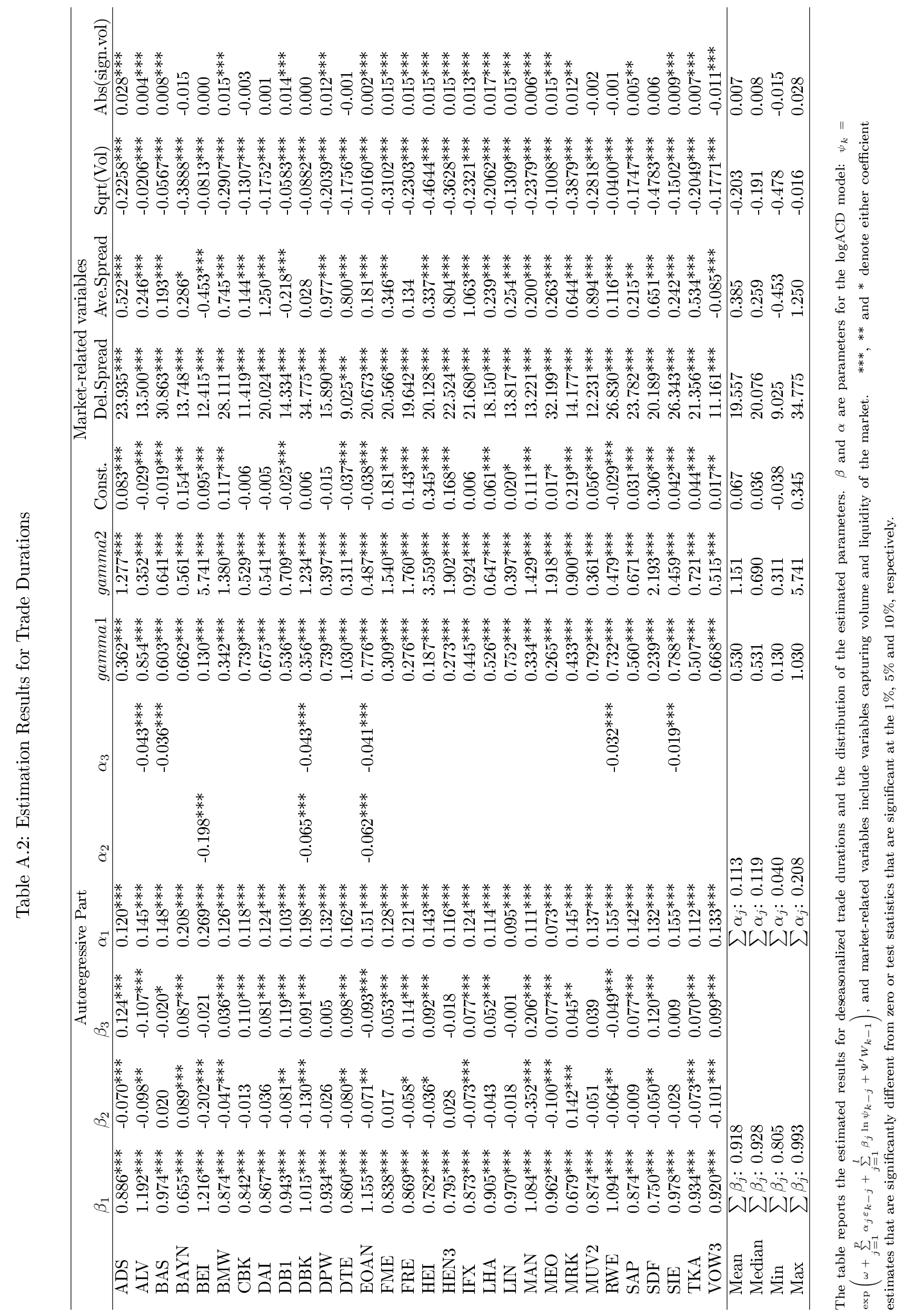


Table A.3: Ljung-boxtest for Trade duration

\begin{tabular}{llll}
\hline Ticker & LB(5) & LB(10) & LB $(15)$ \\
\hline ADS & 13.62 & 28.07 & 35.66 \\
ALV & 36.02 & 56.36 & 81.30 \\
BAS & 27.28 & 41.91 & 54.51 \\
BAYN & 20.89 & 49.85 & 88.41 \\
BEI & 18.71 & 28.22 & 30.53 \\
BMW & 39.65 & 44.58 & 85.01 \\
CBK & 19.47 & 40.10 & 48.99 \\
DAI & 57.26 & 76.42 & 85.18 \\
DB1 & 12.98 & 35.01 & 58.23 \\
DBK & 61.92 & 83.80 & 110.87 \\
DPW & 34.53 & 54.19 & 65.26 \\
DTE & 14.19 & 20.05 & 36.39 \\
EOAN & 22.10 & 42.32 & 60.94 \\
FME & 10.18 & 19.66 & 24.22 \\
FRE & 7.39 & 12.45 & 22.85 \\
HEI & 4.59 & 13.04 & 20.72 \\
HEN3 & 17.48 & 24.39 & 61.58 \\
IFX & 39.38 & 52.08 & 59.89 \\
LHA & 35.35 & 63.98 & 69.99 \\
LIN & 34.32 & 40.45 & 48.61 \\
MAN & 23.99 & 27.97 & 34.30 \\
MEO & 46.62 & 61.52 & 75.81 \\
MRK & 7.61 & 11.37 & 18.31 \\
MUV2 & 27.59 & 44.73 & 54.15 \\
RWE & 17.87 & 28.98 & 38.89 \\
SAP & 38.93 & 66.36 & 70.73 \\
SDF & 1.92 & 3.37 & 11.02 \\
SIE & 14.24 & 48.84 & 59.89 \\
TKA & 61.36 & 83.06 & 89.23 \\
VOW3 & 9.91 & 29.17 & 35.77 \\
\hline Mean & 25.91 & 41.08 & 54.57 \\
Median & 21.50 & 41.18 & 56.37 \\
Min & 1.92 & 3.37 & 11.02 \\
Max & 61.92 & 83.80 & 110.87 \\
\hline & & &
\end{tabular}

The table reports the results of Ljung-Box statistics on 5, 10 and 15 lagged standardized residuals derived from the model for deseasonalized trade durations. The critical value for $\mathrm{LB}(5), \mathrm{LB}(10)$, and LB(15), are 11.07, 18.30 and 24.99 , respectively. 


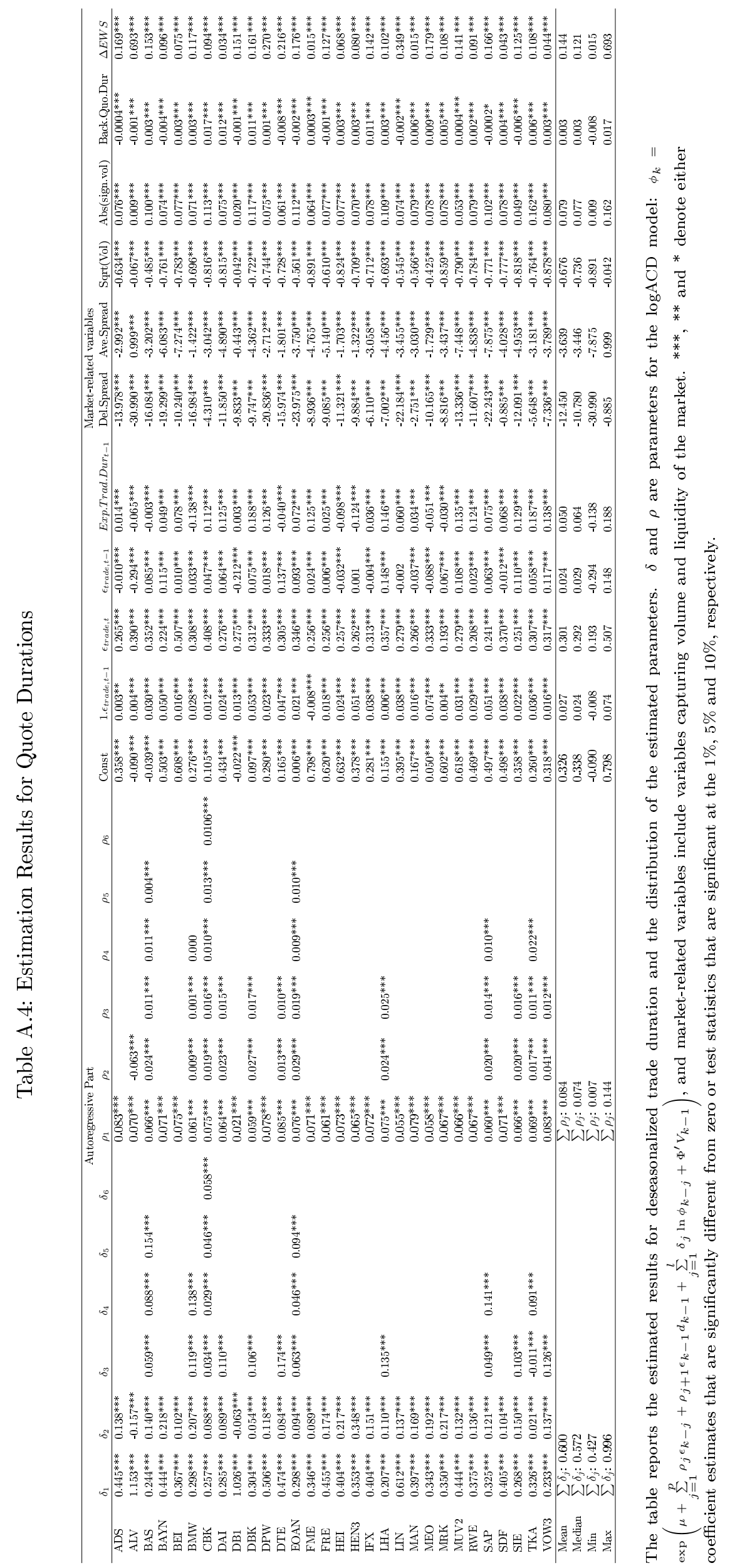


Table A.5: Ljung-box test for Quote Duration

\begin{tabular}{|c|c|c|c|}
\hline Ticker & $\mathrm{LB}(5)$ & $\mathrm{LB}(10)$ & $\mathrm{LB}(15)$ \\
\hline ADS & 24.77 & 46.60 & 62.50 \\
\hline ALV & 49.49 & 69.49 & 72.78 \\
\hline BAS & 3.81 & 9.53 & 26.73 \\
\hline BAYN & 6.02 & 42.13 & 90.68 \\
\hline BEI & 7.19 & 16.88 & 23.01 \\
\hline BMW & 2.54 & 4.51 & 19.22 \\
\hline CBK & 26.78 & 69.83 & 90.18 \\
\hline DAI & 7.63 & 44.47 & 80.00 \\
\hline DB1 & 39.99 & 50.97 & 57.26 \\
\hline DBK & 10.68 & 61.96 & 83.50 \\
\hline DPW & 1.88 & 19.50 & 57.70 \\
\hline DTE & 0.75 & 7.98 & 77.78 \\
\hline EOAN & 1.51 & 22.65 & 88.39 \\
\hline FME & 13.85 & 54.10 & 74.04 \\
\hline FRE & 4.93 & 16.90 & 24.73 \\
\hline HEI & 3.36 & 28.36 & 43.69 \\
\hline HEN3 & 3.93 & 9.42 & 24.82 \\
\hline IFX & 12.92 & 50.75 & 84.54 \\
\hline LHA & 3.54 & 36.88 & 73.94 \\
\hline LIN & 1.65 & 7.33 & 15.89 \\
\hline MAN & 13.87 & 63.08 & 79.31 \\
\hline MEO & 22.06 & 42.32 & 66.42 \\
\hline MRK & 9.21 & 23.43 & 34.71 \\
\hline MUV2 & 7.21 & 24.80 & 48.19 \\
\hline RWE & 13.28 & 46.51 & 84.82 \\
\hline SAP & 0.80 & 9.88 & 59.88 \\
\hline $\mathrm{SDF}$ & 16.41 & 34.65 & 42.82 \\
\hline SIE & 1.24 & 27.91 & 80.59 \\
\hline TKA & 6.65 & 53.16 & 87.39 \\
\hline VOW3 & 0.83 & 35.00 & 60.68 \\
\hline Mean & 10.63 & 34.37 & 60.54 \\
\hline Median & 6.92 & 34.83 & 64.46 \\
\hline Min & 0.75 & 4.51 & 15.89 \\
\hline Max & 49.49 & 69.83 & 90.68 \\
\hline
\end{tabular}

The table reports the results of Ljung-Box statistics on 5, 10 and 15 lagged standardized residuals derived from the model for deseasonalized quote durations. The critical value for $\mathrm{LB}(5), \mathrm{LB}(10)$, and $\mathrm{LB}(15)$, are $11.07,18.30$ and 24.99 respectively. 
Table A.6: Ljung-Box Statistics for Activity, Direction and Size Factors of $E W S^{L o w}$

\begin{tabular}{|c|c|c|c|c|c|c|c|c|c|}
\hline \multirow[b]{2}{*}{ Ticker } & \multicolumn{3}{|l|}{ Activity } & \multicolumn{3}{|l|}{ Direction } & \multicolumn{3}{|l|}{ Size } \\
\hline & $\mathrm{LB}(5)$ & $\mathrm{LB}(10)$ & $\mathrm{LB}(15)$ & $\mathrm{LB}(5)$ & $\mathrm{LB}(10)$ & $\mathrm{LB}(15)$ & $\mathrm{LB}(5)$ & $\mathrm{LB}(10)$ & $\mathrm{LB}(15)$ \\
\hline ADS & 2263.90 & 3013.53 & 3380.36 & 4262.02 & 4272.98 & 4278.42 & 11406.10 & 16103.75 & 19011.01 \\
\hline ALV & 10714.69 & 12349.57 & 13368.93 & 15722.80 & 15732.33 & 15742.48 & 19886.67 & 25562.39 & 30139.48 \\
\hline BAS & 4107.00 & 4820.26 & 5180.01 & 15840.70 & 15869.68 & 15872.90 & 14124.74 & 18961.75 & 22182.30 \\
\hline BAYN & 1937.76 & 2298.52 & 2545.57 & 11216.49 & 11230.11 & 11236.36 & 10188.80 & 14241.93 & 17160.31 \\
\hline BEI & 1004.39 & 1248.55 & 1348.54 & 2382.94 & 2391.76 & 2394.56 & 5514.75 & 7027.23 & 8268.67 \\
\hline BMW & 3556.41 & 3997.27 & 4366.46 & 12238.12 & 12257.00 & 12266.01 & 14236.93 & 18480.90 & 22128.39 \\
\hline CBK & 18053.69 & 21435.57 & 23510.97 & 14967.53 & 14997.56 & 15006.87 & 41313.46 & 56079.61 & 65918.34 \\
\hline DAI & 4654.86 & 5547.40 & 6146.60 & 15327.52 & 15339.90 & 15347.93 & 20517.65 & 27768.33 & 33340.29 \\
\hline DB1 & 3175.01 & 4250.51 & 4957.41 & 5426.88 & 5433.84 & 5443.18 & 17635.42 & 25766.27 & 32212.07 \\
\hline DBK & 7359.92 & 8688.86 & 9470.23 & 18330.57 & 18350.85 & 18359.75 & 28513.96 & 38358.94 & 46838.19 \\
\hline DPW & 5474.27 & 6252.29 & 6628.99 & 5624.87 & 5633.68 & 5637.13 & 6345.21 & 8151.47 & 9277.84 \\
\hline DTE & 15592.69 & 18147.14 & 19849.47 & 7797.13 & 7811.21 & 7814.32 & 13836.69 & 17561.72 & 20360.46 \\
\hline EOAN & 13689.18 & 17370.81 & 19942.26 & 19361.56 & 19368.70 & 19371.93 & 32387.69 & 45657.26 & 56558.42 \\
\hline FME & 1537.04 & 2007.41 & 2313.07 & 3941.67 & 3948.92 & 3951.70 & 14163.88 & 20383.63 & 24706.10 \\
\hline FRE & 1719.88 & 2165.79 & 2437.47 & 2864.18 & 2880.57 & 2888.77 & 14111.19 & 19542.96 & 23078.45 \\
\hline HEI & 3662.05 & 4718.42 & 5407.12 & 5141.84 & 5160.78 & 5177.97 & 16046.19 & 23996.45 & 28985.25 \\
\hline HEN3 & 2219.38 & 2754.75 & 3129.65 & 4612.79 & 4635.70 & 4643.60 & 13847.51 & 19808.52 & 23941.81 \\
\hline IFX & 3665.74 & 4225.39 & 4534.23 & 6867.77 & 6893.79 & 6896.89 & 13943.32 & 18573.85 & 21870.79 \\
\hline LHA & 3964.37 & 4507.99 & 4757.31 & 4458.94 & 4469.85 & 4471.96 & 8879.08 & 12653.70 & 15214.17 \\
\hline LIN & 4232.02 & 5031.44 & 5431.57 & 4265.66 & 4268.82 & 4270.40 & 6235.13 & 8158.98 & 9050.37 \\
\hline MAN & 5437.06 & 7010.95 & 8154.88 & 6815.45 & 6846.41 & 6859.83 & 26909.94 & 42463.55 & 55094.45 \\
\hline MEO & 2650.68 & 3164.90 & 3512.38 & 5463.39 & 5481.36 & 5483.98 & 10071.74 & 13427.03 & 15521.66 \\
\hline MRK & 1637.03 & 2061.70 & 2316.10 & 3938.26 & 3950.28 & 3953.84 & 12645.13 & 17275.60 & 21543.58 \\
\hline MUV2 & 5378.08 & 6008.49 & 6282.16 & 6488.92 & 6493.50 & 6498.73 & 5371.05 & 6641.75 & 7359.34 \\
\hline RWE & 3673.00 & 4368.87 & 4734.41 & 10403.57 & 10421.80 & 10437.37 & 14362.98 & 20931.58 & 26526.60 \\
\hline SAP & 3860.85 & 4507.64 & 4965.08 & 13525.71 & 13541.85 & 13547.17 & 12349.95 & 15928.96 & 18783.85 \\
\hline SDF & 2062.00 & 2469.94 & 2672.47 & 5502.76 & 5509.43 & 5512.05 & 13626.33 & 20052.79 & 24394.20 \\
\hline SIE & 3922.55 & 4484.47 & 4719.65 & 16888.09 & 16901.99 & 16905.70 & 16688.61 & 22366.07 & 26210.41 \\
\hline TKA & 3082.99 & 3593.17 & 3997.47 & 7202.92 & 7229.28 & 7239.13 & 17774.21 & 24979.58 & 30200.80 \\
\hline VOW3 & 7517.48 & 8456.62 & 9012.72 & 5971.91 & 5980.58 & 5981.28 & 10548.54 & 13924.77 & 16366.27 \\
\hline
\end{tabular}

The table reports the Ljung-Box statistic on deseasonalized trade durations, deseasonalized quote durations, activity, direction and size factors of the $E W S^{H i g h}$ for different stocks at 5, 10 and 15 lags. The critical value for $L B(5), L B(10)$, and LB(15) are $11.07,18.30$ and 24.99 , respectively. 


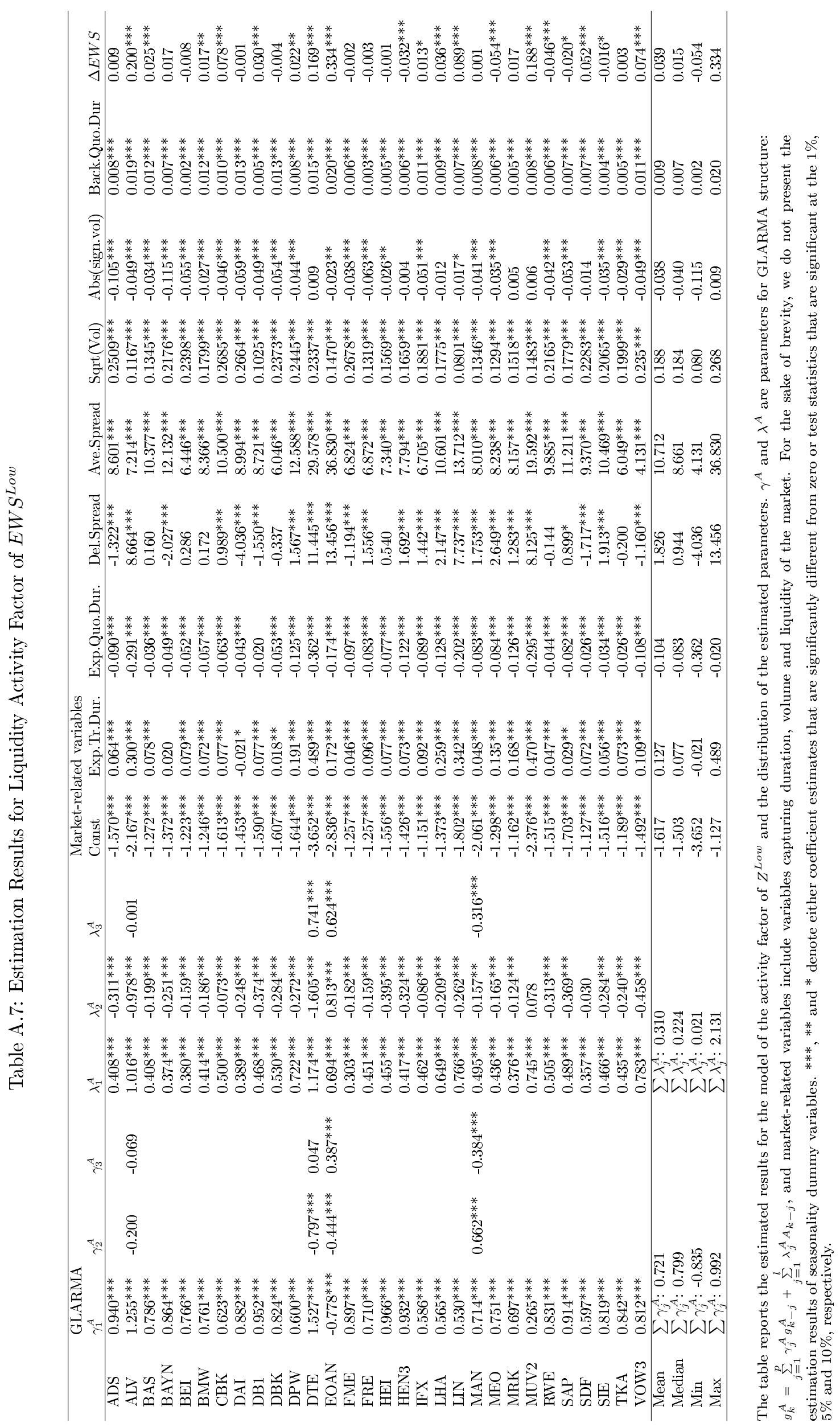


Table A.8: In-sample tests for EWS Activity Factor of $E W S^{\text {low }}$

\begin{tabular}{|c|c|c|c|c|c|c|}
\hline Ticker & $\mathrm{LB}(5)$ & $\mathrm{LB}(10)$ & LB(15) & R-sqrt & $\mathrm{ROC}$ & \#_Acc \\
\hline ADS & 44.021 & 48.040 & 59.783 & $19.83 \%$ & 0.618 & $59.16 \%$ \\
\hline ALV & 26.403 & 64.356 & 92.971 & $40.37 \%$ & 0.719 & $69.70 \%$ \\
\hline BAS & 9.526 & 11.952 & 13.915 & $27.90 \%$ & 0.589 & $60.57 \%$ \\
\hline BAYN & 3.320 & 6.384 & 7.879 & $24.47 \%$ & 0.586 & $58.80 \%$ \\
\hline BEI & 2.891 & 3.842 & 5.091 & $14.29 \%$ & 0.591 & $57.93 \%$ \\
\hline BMW & 10.397 & 11.119 & 22.330 & $22.20 \%$ & 0.595 & $58.05 \%$ \\
\hline CBK & 9.730 & 24.582 & 62.335 & $22.93 \%$ & 0.642 & $60.76 \%$ \\
\hline DAI & 11.103 & 16.697 & 22.586 & $23.40 \%$ & 0.600 & $59.01 \%$ \\
\hline DB1 & 50.529 & 51.917 & 57.842 & $21.70 \%$ & 0.631 & $59.91 \%$ \\
\hline DBK & 23.452 & 29.111 & 39.509 & $32.33 \%$ & 0.612 & $63.64 \%$ \\
\hline DPW & 10.376 & 19.499 & 28.645 & $19.55 \%$ & 0.657 & $62.25 \%$ \\
\hline DTE & 52.705 & 72.464 & 94.669 & $46.07 \%$ & 0.769 & $72.49 \%$ \\
\hline EOAN & 42.447 & 58.580 & 87.181 & $44.65 \%$ & 0.717 & $70.70 \%$ \\
\hline FME & 8.017 & 12.805 & 19.908 & $13.97 \%$ & 0.610 & $59.30 \%$ \\
\hline FRE & 3.084 & 4.663 & 11.829 & $17.67 \%$ & 0.621 & $59.27 \%$ \\
\hline HEI & 56.213 & 57.060 & 58.825 & $19.25 \%$ & 0.619 & $59.07 \%$ \\
\hline HEN3 & 27.584 & 31.653 & 39.311 & $20.60 \%$ & 0.608 & $58.58 \%$ \\
\hline IFX & 8.232 & 19.780 & 34.010 & $20.60 \%$ & 0.604 & $58.17 \%$ \\
\hline LHA & 9.985 & 17.112 & 22.136 & $18.75 \%$ & 0.654 & $61.46 \%$ \\
\hline LIN & 11.806 & 23.285 & 25.782 & $23.56 \%$ & 0.690 & $64.31 \%$ \\
\hline MAN & 11.778 & 19.493 & 25.860 & $27.86 \%$ & 0.632 & $62.17 \%$ \\
\hline MEO & 3.970 & 11.300 & 18.178 & $24.10 \%$ & 0.611 & $59.52 \%$ \\
\hline MRK & 2.757 & 7.157 & 14.726 & $18.15 \%$ & 0.608 & $58.22 \%$ \\
\hline MUV2 & 12.585 & 18.240 & 21.078 & $26.06 \%$ & 0.711 & $65.69 \%$ \\
\hline RWE & 11.788 & 22.205 & 23.118 & $30.03 \%$ & 0.604 & $62.12 \%$ \\
\hline SAP & 18.729 & 23.372 & 26.166 & $34.62 \%$ & 0.600 & $65.55 \%$ \\
\hline SDF & 3.643 & 8.764 & 21.495 & $16.08 \%$ & 0.608 & $58.30 \%$ \\
\hline SIE & 6.009 & 9.700 & 26.749 & $31.96 \%$ & 0.588 & $63.65 \%$ \\
\hline TKA & 29.774 & 36.151 & 38.478 & $20.90 \%$ & 0.598 & $57.84 \%$ \\
\hline VOW3 & 20.357 & 21.744 & 26.612 & $20.31 \%$ & 0.658 & $62.10 \%$ \\
\hline Mean & 18.107 & 25.434 & 34.967 & $24.81 \%$ & 0.632 & $61.61 \%$ \\
\hline Median & 11.440 & 19.640 & 26.013 & $22.57 \%$ & 0.611 & $60.24 \%$ \\
\hline Min & 2.757 & 3.842 & 5.091 & $13.97 \%$ & 0.586 & $57.84 \%$ \\
\hline Max & 56.213 & 72.464 & 94.669 & $46.07 \%$ & 0.769 & $72.49 \%$ \\
\hline
\end{tabular}

The table reports the results of in-sample tests for the activity factor model. LB(5), LB(10) and LB(15) are Ljung-Box statistics on 5, 10 and 15 lagged standardized residuals. The critical value for $\mathrm{LB}(5), \mathrm{LB}(10)$, and $\mathrm{LB}(15)$, are 11.07 , 18.30 and 24.99, respectively. R-sqrt is the McFadden's R squared, defined as $R_{M c F a d e n}^{2}=1-\frac{\log \left(L_{c}\right)}{\log \left(L_{m}\right)}$ where $L_{c}$ denotes the likelihood value from the current fitted model and $L_{n u l l}$ denotes the corresponding value for the null model. ROC relates the Receiver Operating Characteristic test. \#_Acc is the Count accuracy that takes $50 \%$ as the threshold to have value one. 


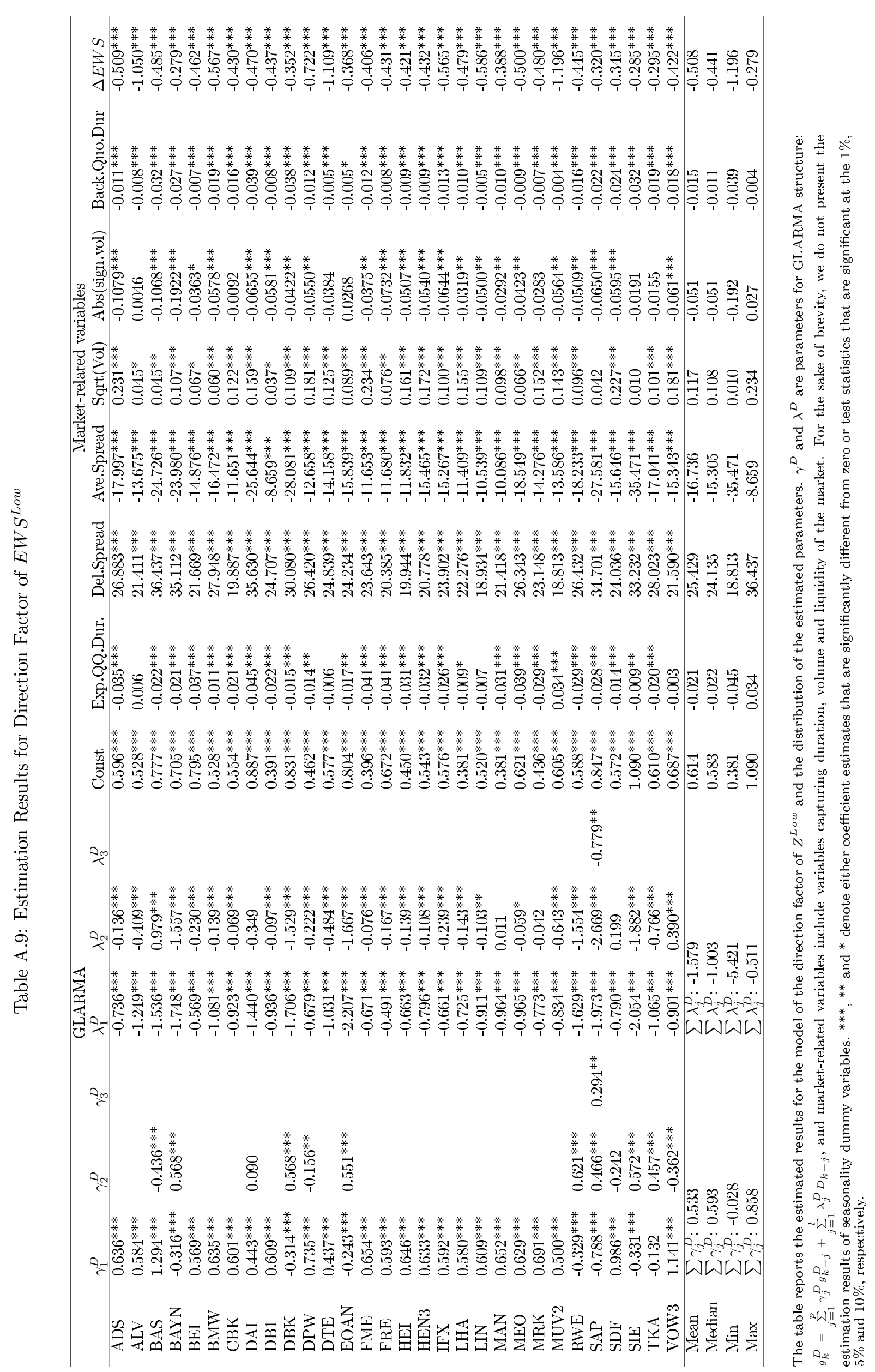


Table A.10: In-sample tests for EWS Direction Factor of EW $S^{\text {low }}$

\begin{tabular}{lllllll}
\hline Ticker & LB(5) & LB(10) & LB(15) & R-sqrt & ROC & \#_Acc \\
\hline ADS & 42.628 & 49.748 & 58.858 & $36.54 \%$ & 0.807 & $74.48 \%$ \\
ALV & 58.857 & 71.547 & 77.410 & $50.79 \%$ & 0.875 & $82.83 \%$ \\
BAS & 8.501 & 49.369 & 64.249 & $42.50 \%$ & 0.852 & $78.27 \%$ \\
BAYN & 9.944 & 23.216 & 29.500 & $44.45 \%$ & 0.864 & $79.53 \%$ \\
BEI & 5.442 & 10.754 & 12.848 & $33.26 \%$ & 0.792 & $73.08 \%$ \\
BMW & 9.872 & 29.203 & 59.222 & $38.86 \%$ & 0.825 & $76.25 \%$ \\
CBK & 42.181 & 69.001 & 72.996 & $35.43 \%$ & 0.808 & $74.66 \%$ \\
DAI & 9.529 & 16.674 & 25.804 & $43.16 \%$ & 0.852 & $78.19 \%$ \\
DB1 & 33.289 & 55.562 & 57.286 & $35.32 \%$ & 0.810 & $75.05 \%$ \\
DBK & 25.728 & 33.059 & 39.995 & $43.76 \%$ & 0.860 & $79.17 \%$ \\
DPW & 22.580 & 33.983 & 38.533 & $42.64 \%$ & 0.845 & $78.41 \%$ \\
DTE & 8.191 & 13.944 & 16.673 & $52.84 \%$ & 0.874 & $83.92 \%$ \\
EOAN & 15.452 & 38.557 & 44.336 & $60.25 \%$ & 0.917 & $87.75 \%$ \\
FME & 21.008 & 28.535 & 34.325 & $34.04 \%$ & 0.795 & $73.08 \%$ \\
FRE & 17.067 & 25.496 & 28.599 & $31.10 \%$ & 0.773 & $71.05 \%$ \\
HEI & 5.734 & 39.836 & 47.088 & $32.21 \%$ & 0.786 & $72.50 \%$ \\
HEN3 & 20.853 & 38.412 & 45.239 & $33.34 \%$ & 0.794 & $73.41 \%$ \\
IFX & 24.274 & 50.654 & 71.168 & $35.43 \%$ & 0.803 & $73.85 \%$ \\
LHA & 7.506 & 9.976 & 17.584 & $36.59 \%$ & 0.810 & $74.65 \%$ \\
LIN & 8.118 & 14.480 & 23.097 & $39.95 \%$ & 0.832 & $77.84 \%$ \\
MAN & 0.244 & 2.215 & 7.754 & $34.27 \%$ & 0.799 & $74.25 \%$ \\
MEO & 15.223 & 47.557 & 54.420 & $36.83 \%$ & 0.814 & $75.66 \%$ \\
MRK & 26.733 & 60.896 & 71.922 & $34.10 \%$ & 0.800 & $73.53 \%$ \\
MUV2 & 49.866 & 53.084 & 53.447 & $50.81 \%$ & 0.875 & $82.86 \%$ \\
RWE & 43.513 & 55.877 & 68.253 & $44.01 \%$ & 0.856 & $79.58 \%$ \\
SAP & 69.833 & 84.509 & 97.393 & $47.87 \%$ & 0.879 & $81.35 \%$ \\
SDF & 1.827 & 5.635 & 7.272 & $33.54 \%$ & 0.801 & $73.02 \%$ \\
SIE & 23.971 & 28.765 & 36.255 & $46.15 \%$ & 0.871 & $80.06 \%$ \\
TKA & 2.409 & 4.320 & 23.224 & $34.84 \%$ & 0.808 & $73.85 \%$ \\
VOW3 & 33.542 & 41.788 & 47.014 & $38.37 \%$ & 0.825 & $76.04 \%$ \\
\hline Mean & 22.131 & 36.222 & 44.392 & $40.11 \%$ & 0.830 & $76.94 \%$ \\
Median & 18.960 & 36.197 & 44.787 & $37.60 \%$ & 0.819 & $75.85 \%$ \\
Min & 0.244 & 2.215 & 7.272 & $31.10 \%$ & 0.773 & $71.05 \%$ \\
\hline & 69.833 & 84.509 & 97.393 & $60.25 \%$ & 0.917 & $87.75 \%$ \\
\hline & & & & & &
\end{tabular}

The table reports the results of in-sample tests for direction model. $\mathrm{LB}(5), \mathrm{LB}(10)$ and $\mathrm{LB}(15)$ are Ljung-Box statistics on 5, 10 and 15 lagged standardized residuals. The critical value for $\mathrm{LB}(5), \mathrm{LB}(10)$, and $\mathrm{LB}(15)$, are $11.07,18.30$ and 24.99 , respectively. R-sqrt is the McFadden's R squared, defined as $R_{\text {McFaden }}^{2}=1-\frac{\log \left(L_{c}\right)}{\log \left(L_{m}\right)}$ where $L_{c}$ denotes the likelihood value from the current fitted model and $L_{n u l l}$ denotes the corresponding value for the null model. ROC relates the Receiver Operating Characteristic test. \#_Acc is the Count accuracy that takes 50\% as the threshold to have value one. 


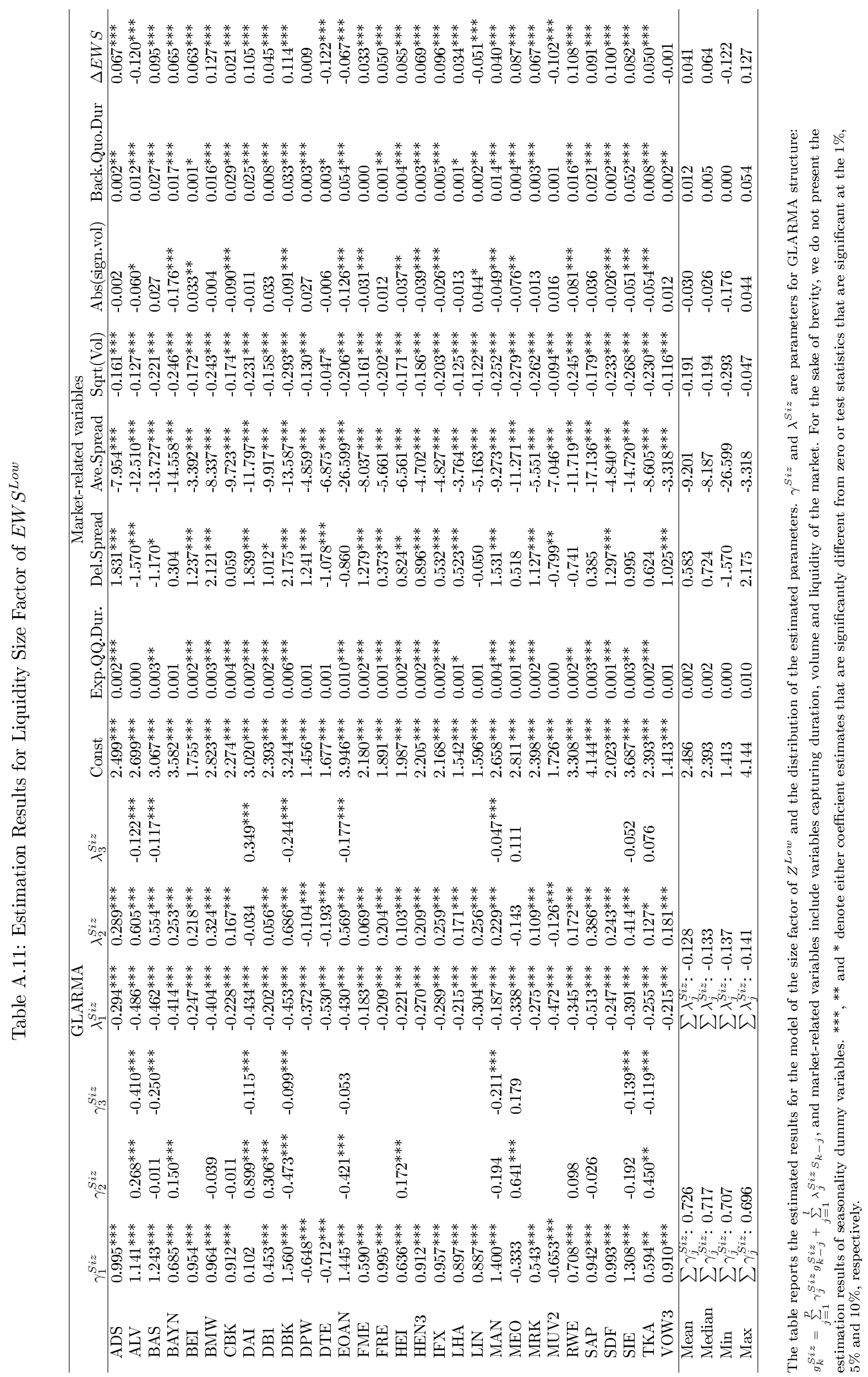


Table A.12: Ljung-Box Statistics for EWS Size Factor of EW $S^{\text {low }}$

\begin{tabular}{lllll}
\hline Ticker & LB(5) & LB(10) & LB(15) & R-sqrt \\
\hline ADS & 175.50 & 221.53 & 241.47 & $22.53 \%$ \\
ALV & 274.78 & 313.31 & 365.32 & $35.20 \%$ \\
BAS & 255.43 & 299.16 & 335.00 & $36.19 \%$ \\
BAYN & 234.61 & 246.84 & 277.05 & $15.46 \%$ \\
BEI & 93.74 & 104.50 & 126.03 & $48.62 \%$ \\
BMW & 223.63 & 269.45 & 284.82 & $43.46 \%$ \\
CBK & 168.83 & 181.96 & 205.73 & $50.32 \%$ \\
DAI & 280.53 & 292.45 & 310.84 & $47.18 \%$ \\
DB1 & 222.08 & 243.01 & 268.12 & $50.02 \%$ \\
DBK & 358.91 & 411.44 & 474.05 & $61.85 \%$ \\
DPW & 47.11 & 74.02 & 88.84 & $14.59 \%$ \\
DTE & 63.85 & 94.66 & 110.36 & $27.80 \%$ \\
EOAN & 443.41 & 470.93 & 496.03 & $26.90 \%$ \\
FME & 206.02 & 247.10 & 273.44 & $38.98 \%$ \\
FRE & 63.21 & 105.58 & 117.23 & $55.08 \%$ \\
HEI & 184.26 & 204.58 & 269.54 & $51.62 \%$ \\
HEN3 & 234.06 & 265.84 & 301.73 & $4.27 \%$ \\
IFX & 185.20 & 219.44 & 244.39 & $8.49 \%$ \\
LHA & 116.35 & 125.76 & 133.11 & $43.47 \%$ \\
LIN & 105.67 & 109.16 & 116.94 & $16.04 \%$ \\
MAN & 522.77 & 654.96 & 766.09 & $60.06 \%$ \\
MEO & 122.52 & 136.15 & 150.95 & $51.15 \%$ \\
MRK & 172.85 & 223.75 & 255.55 & $38.80 \%$ \\
MUV2 & 73.88 & 93.93 & 125.45 & $39.14 \%$ \\
RWE & 275.40 & 307.52 & 315.93 & $42.43 \%$ \\
SAP & 211.65 & 240.69 & 279.33 & $19.71 \%$ \\
SDF & 192.43 & 233.25 & 251.95 & $34.59 \%$ \\
SIE & 408.45 & 466.75 & 506.98 & $48.78 \%$ \\
TKA & 319.61 & 353.24 & 417.10 & $54.58 \%$ \\
VOW3 & 184.67 & 200.91 & 232.00 & $58.55 \%$ \\
\hline Mean & 214.05 & 247.06 & 278.05 & $38.20 \%$ \\
Median & 199.23 & 236.97 & 268.83 & $40.78 \%$ \\
Min & 47.11 & 74.02 & 88.84 & $4.27 \%$ \\
Max & 522.77 & 654.96 & 766.09 & $61.85 \%$ \\
\hline & & & &
\end{tabular}

The table reports the results of Ljung-Box statistics on 5, 10 and 15 lagged standardized residuals and adjusted $R^{2}$. The critical value for $\mathrm{LB}(5), \mathrm{LB}(10)$, and $\mathrm{LB}(15)$, are $11.07,18.30$ and 24.99 , respectively. 